quatrième série-tome 41 fascicule 2 mars-avril 2008

$$
\begin{aligned}
& \text { ANNALES } \\
& \text { SCIENTIFIQUES } \\
& \text { de } \\
& \text { L'ECOLE } \\
& \text { NORMALE } \\
& \text { SUPERIEURE }
\end{aligned}
$$

Ferruccio COLOMBINI \& Guy MÉTIVIER

The Cauchy Problem for Wave Equations

with non Lipschitz Coefficients 


\title{
THE CAUCHY PROBLEM FOR WAVE EQUATIONS WITH NON LIPSCHITZ COEFFICIENTS; APPLICATION TO CONTINUATION OF SOLUTIONS
OF SOME NONLINEAR WAVE EQUATIONS
}

\author{
BY FERRUCCIO COLOMBINI AND GuY MÉTIVIER
}

\begin{abstract}
In this paper we study the Cauchy problem for second order strictly hyperbolic operators of the form

$$
L u:=\sum_{j, k=0}^{n} \partial_{y_{j}}\left(a_{j, k} \partial_{y_{k}} u\right)+\sum_{j=0}^{n}\left\{b_{j} \partial_{y_{j}} u+\partial_{y_{j}}\left(c_{j} u\right)\right\}+d u=f
$$

when the coefficients of the principal part are not Lipschitz continuous, but only "Log-Lipschitz" with respect to all the variables. This class of equation is invariant under changes of variables and therefore suitable for a local analysis. In particular, we show local existence, local uniqueness and finite speed of propagation for the noncharacteristic Cauchy problem. This provides an invariant version of a previous paper of the first author with N. Lerner [6]. We also give an application of the method to a continuation theorem for nonlinear wave equations where the coefficients above depend on $u$ : the smooth solution can be extended as long as it remains Log-Lipschitz.
\end{abstract}

RÉsumÉ. - On considère le problème de Cauchy pour des équations d'onde strictement hyperboliques :

$$
L u:=\sum_{j, k=0}^{n} \partial_{y_{j}}\left(a_{j, k} \partial_{y_{k}} u\right)+\sum_{j=0}^{n}\left\{b_{j} \partial_{y_{j}} u+\partial_{y_{j}}\left(c_{j} u\right)\right\}+d u=f
$$

quand les coefficients de la partie principale sont seulement "Log-Lipschitz" en toutes les variables. Cette classe d'équation est invariante par changement de variables et est donc une classe naturelle pour une étude locale intrinsèque. En particulier, on montre l'existence locale, l'unicité locale et la vitesse finie de propagation pour le problème de Cauchy non caractéristique, donnant une version invariante d'un résultat antérieur du premier auteur avec N. Lerner [6]. Pour les équations non linéaires où les coefficients ci-dessus dépendent de $u$, la méthode d'estimations permet de montrer que les solutions régulières se prolongent en solutions régulières aussi longtemps qu'elles restent Log-Lipschitz.

\section{Introduction}

In this paper we study the well-posedness of the Cauchy problem for second order strictly hyperbolic equations whose coefficients are not Lipschitz continuous:

$$
L u:=\sum_{j, k=0}^{n} \partial_{y_{j}}\left(a_{j, k} \partial_{y_{k}} u\right)+\sum_{j=0}^{n}\left\{b_{j} \partial_{y_{j}} u+\partial_{y_{j}}\left(c_{j} u\right)\right\}+d u=f .
$$


In Section 6, we will present an application of the methods developed for the analysis of the Cauchy problem to nonlinear wave equations, where the various coefficients above depend on $u$. It is known that the smooth solution can be extended as long as they remain Lipschitz continuous. We prove that this condition can be weakened, and that smooth solutions remain smooth as long as they remain Log-Lipschitz. We refer to Section 6 for a precise result and focus now on the analysis of the Cauchy problem.

The question of the well-posedness of the Cauchy problem for the wave equation with nonsmooth coefficients has already been studied in the case that the second order part has the special form, in coordinates $y=(t, x)$ :

$$
\partial_{t}^{2}-\sum_{j, k=1}^{n} \partial_{x_{j}}\left(a_{j, k} \partial_{x_{k}} u\right)
$$

and the Cauchy data are given on the space-like hyperplane $\{t=0\}$. In this case, when the coefficients depend only on the time variable $t$, F. Colombini, E. De Giorgi and S. Spagnolo ([5]) have proved that the Cauchy problem is in general ill-posed in $C^{\infty}$ when the coefficients are only Hölder continuous of order $\alpha<1$, but is well-posed in appropriate Gevrey spaces. This has been extended to the case where the coefficients are Hölder in time and Gevrey in $x$ ([14, 8]). Moreover, it is also proved in [5] that the Cauchy problem is well posed in $C^{\infty}$ when the coefficients, which depend only on time, are "Log-Lipschitz" (in short LL) : recall that a function $a$ of variables $y$ is said to be LL on a domain $\Omega$ if there is a constant $C$ such that

$$
\left|a(y)-a\left(y^{\prime}\right)\right| \leq C\left|y-y^{\prime}\right|\left(1+|\log | y-y^{\prime}||\right)
$$

for all $y$ and $y^{\prime}$ in $\Omega$. In [5], it is proved that for LL coefficients depending only on $t$ and for initial data in the Sobolev spaces $H^{s} \times H^{s-1}$, the solution satisfies

$$
u(t, \cdot) \in H^{s-\lambda t}, \quad \partial_{t} u(t, \cdot) \in H^{s-1-\lambda t}
$$

with $\lambda$ depending only on the $L L$ norms of the coefficients and the constants of hyperbolicity. In particular, there is a loss of smoothness as time evolves and this loss does occur in general when the coefficients are not Lipschitz continuous, and is sharp, as shown in [3].

The analysis of the $C^{\infty}$ well-posedness has been extended by F. Colombini and N. Lerner ([6]) to the case of equations, still with principal part (1.2), whose coefficients also depend on the space variables $x$. They show that the Cauchy problem is well-posed if the coefficients are LL in time and $C^{\infty}$ in $x$. They also study the problem under the natural assumption of isotropic LL smoothness in $(t, x)$. In this case one has to multiply LL functions with distributions in $H^{s}$. This is well defined only when $|s|<1$. Therefore, one considers initial data in $H^{s} \times H^{s-1}$ with $0<s<1$, noticing that further smoothness would not help. Next, the loss of smoothness (1.4) forces us to limit $t$ to an interval where $0<s-\lambda t$, yielding only local in time existence theorems. We also refer to [6] for further discussions on the sharpness of LL smoothness.

However, the local uniqueness of the Cauchy problem and the finite speed of propagation for local solutions are not proved in [6]. The main goal of this paper is to address these questions. Classical methods, such as convexification, lead one to consider general equations (1.1) with LL coefficients in all variables. However, the meaning of the Cauchy problem for such equations is not completely obvious: as mentioned above, the maximal expected smoothness 
of the solutions is $H^{s}$ with $s<1$ and their traces on the initial manifold are not immediately defined. More importantly, in the general theory of smooth operators, the traces are defined using partial regularity results in the normal direction; in our case, the limited smoothness of the coefficients is a source of difficulties. It turns out that when $s \leq \frac{1}{2}$, one cannot in general define the traces of all the first order derivatives of $u$, but only the Neumann trace relative to the operator, using a weak formulation of the traces.

Assumption 1.1. - $L$ is a second order operator of the form (1.1) on a neighborhood $\Omega$ of $y$, with coefficients $a_{j, k} \in L L(\Omega), b_{j}$ and $c_{j}$ in $C^{\alpha}(\Omega)$, for some $\left.\alpha \in\right] \frac{1}{2}, 1\left[\right.$ and $d \in L^{\infty}(\Omega)$. $\Sigma$ is a smooth hypersurface through $y$ and $L$ is strictly hyperbolic in the direction conormal to $\Sigma$.

Shrinking $\Omega$ if necessary, we assume that $\Sigma$ is defined by the equation $\{\varphi=0\}$ with $\varphi$ smooth and $d \varphi \neq 0$. We consider the one-sided Cauchy problem, say on the component $\Omega_{+}=\Omega \cap\{\varphi>0\}$. We use the Sobolev spaces $H^{s}(\Omega \cap\{\varphi>0\})$ for $s \in \mathbb{R}$. As usual, we say that $u \in H_{\text {loc }}^{s}(\Omega \cap\{\varphi \geq 0\})$, if for any relatively compact open subset $\Omega_{1}$ of $\Omega$, the restriction of $u$ to $\Omega_{1} \cap\{\varphi>0\}$ belongs to $H^{s}(\Omega \cap\{\varphi>0\})$. Similarly, $u \in H_{\text {comp }}^{s}(\Omega \cap\{\varphi \geq 0\})$ if $u \in H^{s}(\Omega \cap\{\varphi>0\})$ has compact support in $\Omega \cap\{\varphi \geq 0\}$.

The adjoint operator

$$
L^{*} v:=\sum_{j, k=0}^{n} \partial_{y_{k}}\left(\bar{a}_{j, k} \partial_{y_{j}} v\right)-\sum_{j=0}^{n}\left\{\bar{c}_{j} \partial_{y_{j}} v+\partial_{y_{j}}\left(\bar{b}_{j} v\right)\right\}+\bar{d} v
$$

has the same form as $L$. For $u$ and $v$ smooth, $v$ compactly supported in $\Omega \cap\{\varphi \geq 0\}$, one has the (formal) identity

$$
(L u, v)_{L^{2}\left(\Omega_{+}\right)}-\left(u, L^{*} v\right)_{L^{2}\left(\Omega_{+}\right)}=\left(N_{\nu} u, v\right)_{L^{2}(\Sigma)}-\left(u, N_{\nu}^{\prime} v\right)_{L^{2}(\Sigma)}
$$

where

$$
\begin{aligned}
& N_{\nu} u=\sum_{j, k} \nu_{k}\left(a_{j, k} \partial_{j} u\right)_{\mid \Sigma}, \\
& N_{\nu}^{\prime} v=\sum_{j, k} \nu_{j}\left(\bar{a}_{j, k} \partial_{k} v\right)_{\mid \Sigma}-\sum_{j} \nu_{j}\left(\left(\bar{b}_{j}+\bar{c}_{j}\right) v\right)_{\mid \Sigma}
\end{aligned}
$$

and $\nu=\left(\nu_{0}, \ldots, \nu_{d}\right) \neq 0$ is conormal to $\Sigma$ and the $d$-integration form on $\Sigma$ is chosen accordingly.

Lemma 1.2. - $\quad$ i) For all $s \in] 1-\alpha, 1+\alpha\left[\right.$ and $u \in H_{\text {loc }}^{s}(\Omega \cap\{\varphi \geq 0\})$, all the terms entering in the definition of $L u$ and $L^{*} u$ are well defined as distributions in $H_{\text {loc }}^{s-2}(\Omega \cap\{\varphi \geq 0\})$.

ii) For all $s \in] \frac{3}{2}, 1+\alpha\left[\right.$ and $u \in H_{l o c}^{s}(\Omega \cap\{\varphi \geq 0\})$, the traces $N_{\nu} u$ and $N_{\nu}^{\prime} u$ are well defined in $H_{l o c}^{s-\frac{3}{2}}(\Sigma \cap \Omega)$.

Proof. - This is due to multiplicative properties (see [6] and Corollary 3.6):

- If $\sigma \in]-1,1\left[, a \in L L(\Omega)\right.$ and $v \in H_{l o c}^{\sigma}(\Omega \cap\{\varphi \geq 0\})$, then $a v \in H_{l o c}^{\sigma}(\Omega \cap\{\varphi \geq 0\})$.

- If $\sigma \in]-\alpha, \alpha\left[, a \in C^{\alpha}(\Omega)\right.$ and $v \in H_{l o c}^{\sigma}(\Omega \cap\{\varphi \geq 0\})$, then $a v \in H_{l o c}^{\sigma}(\Omega \cap\{\varphi \geq 0\})$. 
Next, we recall that the subspace of functions with compact support in $\Omega_{+}$is dense in $H^{\sigma}\left(\Omega_{+}\right)$when $|\sigma|<\frac{1}{2}$; moreover, for $0 \leq \sigma<\frac{1}{2}$ and for $u \in H^{\sigma}\left(\Omega_{+}\right)$the pairing $(u, v)_{L^{2}(\Omega)}$ for $v \in L^{2}$ extends as the duality $\langle u, v\rangle_{H^{\sigma} \times H^{-\sigma}}$. With this remark in mind, the identity (1.6) holds for smooth functions:

Lemma 1.3. - For $s \in] \frac{3}{2}, 1+\alpha\left[, u \in H_{\text {loc }}^{s}(\Omega \cap\{\varphi \geq 0\})\right.$ and $v \in H_{\text {comp }}^{s}(\Omega \cap\{\varphi \geq 0\})$, there holds

$$
\langle L u, v\rangle_{H^{-\sigma} \times H^{\sigma}}-\left\langle u, L^{*} v\right\rangle_{H^{\sigma} \times H^{-\sigma}}=\left(N_{\nu} u, D_{\Sigma} v\right)_{L^{2}(\Sigma)}-\left(D_{\Sigma} u, N_{\nu}^{\prime} v\right)_{L^{2}(\Sigma)}
$$

with $\left.\sigma=s-\frac{3}{2} \in\right] 0, \frac{1}{2}\left[\right.$ and $D_{\Sigma} u=u_{\mid \Sigma}$.

Proof. - It is sufficient to remark that for $\sigma \in\left[0, \frac{1}{2}[\right.$, the Green's formula

$$
\left\langle\partial_{j} u, v\right\rangle_{H^{-\sigma} \times H^{\sigma}}=-\left\langle u, \partial_{j} v\right\rangle_{H^{\sigma} \times H^{-\sigma}}+\left(\nu_{j} D_{\Sigma} u, D_{\Sigma} v\right)_{L^{2}(\Sigma)}
$$

is satisfied for $u \in H_{l o c}^{1-\sigma}(\Omega \cap\{\varphi \geq 0\})$ and $v \in H_{\text {comp }}^{1-\sigma}(\Omega \cap\{\varphi \geq 0\})$.

Proposition 1.4. - Let $D\left(L ; H^{s}\right)=\left\{u \in H_{\text {loc }}^{s}(\Omega \cap\{\varphi \geq 0\}): L u \in L_{\text {loc }}^{2}(\Omega \cap\right.$ $\{\varphi \geq 0\})\}$. The operator $N_{\Sigma}$ and $D_{\Sigma}$ have unique extensions to $\bigcup_{s>1-\alpha} D\left(L ; H^{s}\right)$ such that

i) For all $s \in] 1-\alpha, \alpha\left[, N_{\Sigma}\right.$ (resp. $D_{\Sigma}$ ) is continuous from $D\left(L ; H^{s}\right)$ into $H_{l o c}^{s-\frac{3}{2}}(\Sigma \cap \Omega)$ (resp. $H_{l o c}^{s-\frac{1}{2}}(\Sigma \cap \Omega)$ ).

ii) for all $\left.s^{\prime} \in\right] 1-\alpha, \frac{1}{2}\left[\right.$ such that $s^{\prime} \leq s$ and all $v \in H_{\text {comp }}^{2-s^{\prime}}(\Omega \cap\{\varphi \geq 0\})$ there holds

$$
(L u, v)_{L^{2}}-\left\langle u, L^{*} v\right\rangle_{H^{s^{\prime}} \times H^{-s^{\prime}}}=\left\langle N_{\nu} u, D_{\Sigma} v\right\rangle_{H^{s-\frac{3}{2}} \times H^{\frac{3}{2}-s}}-\left\langle D_{\Sigma} u, N_{\nu}^{\prime} v\right\rangle_{H^{s-\frac{1}{2}} \times H^{\frac{1}{2}-s}} .
$$

This proposition is proved in Section 5. Note that by Lemma 1.2, for $v \in H_{\text {comp }}^{2-s^{\prime}}, L^{*} v \in$ $H_{\text {comp }}^{-s^{\prime}}$ and that $u \in H_{l o c}^{s^{\prime}}$ if $s^{\prime} \leq s$. Moreover, $D_{\Sigma} v \in H_{\text {comp }}^{\frac{3}{2}-s^{\prime}} \subset H_{\text {comp }}^{\frac{3}{2}-s}$ and $N_{\Sigma}^{\prime} v \in H_{\text {comp }}^{\frac{1}{2}-s^{\prime}} \subset$ $H_{\text {comp. }}^{\frac{1}{2}-s}$

With this proposition, the Cauchy problem with source term in $L^{2}$ and solution in $H^{s}$, $s>1-\alpha$, makes sense.

Theorem 1.5 (Local existence). - Consider $s>1-\alpha$ and a neigborhood $\omega$ of $y$ in $\Sigma$. Then there are $\left.s^{\prime} \in\right] 1-\alpha, \alpha\left[\right.$ and a neighborhood $\Omega^{\prime}$ of $y$ in $\mathbb{R}^{1+n}$ such that for all Cauchy data $\left(u_{0}, u_{1}\right)$ in $H^{s}(\omega) \times H^{s-1}(\omega)$ near y and all $f \in L^{2}\left(\Omega^{\prime} \cap\{\varphi>0\}\right)$ the Cauchy problem

$$
L u=f, \quad D_{\Sigma} u=u_{0}, \quad N_{\Sigma} u=u_{1},
$$

has a solution $u \in H^{s^{\prime}}\left(\Omega^{\prime} \cap\{\varphi>0\}\right)$.

Theorem 1.6 (Local uniqueness). - If $s>1-\alpha$ and $u \in H^{s}(\Omega \cap\{\varphi>0\})$ satisfies

$$
L u=0, \quad D_{\Sigma} u=0, \quad N_{\Sigma} u=0
$$

then $u=0$ on a neighborhood of $y$ in $\Omega \cap\{\varphi \geq 0\}$.

REMARK 1.7. - If the coefficients of the first order term $L_{1}$ (see (2.3)) are also $L L$, the statements above are true with $\alpha=1$ since the coefficients are then $C^{\alpha}$ for all $\alpha<1$. If the $b_{j}$ are $C^{\alpha}$ and the $c_{j}$ are $C^{\tilde{\alpha}}$, the conditions are $1-\tilde{\alpha}<\alpha$ and the limitation on $s$ is $1-\tilde{\alpha}<s$. 
REMARK 1.8. - Theorem 1.6 implies that if $u$ is in $H^{s}$ and satisfies $L u=0$ near $y$ and if $u$ vanishes on $\{\varphi<0\}$, then $u$ vanishes on a neighborhood of $y$ (see Section 5.2). Moreover, this local propagation of zero across any space-like manifold implies finite speed of propagation by classical arguments which we do not repeat here. In particular, if $\Omega^{\prime} \cap\{\varphi \geq 0\}$ is contained in the domain of dependence of $\omega$, there is existence and uniqueness for the Cauchy problem (1.10) in $\Omega^{\prime} \cap\{\varphi \geq 0\}$.

The proof of these results is given in Section 5 below. Because all the hypotheses are invariant under smooth changes of coordinates, we can assume that in the coordinates $y=(t, x)$, the initial surface is $\{t=0\}$, and in these coordinates, we prove the existence and uniqueness theorems. We deduce them from similar results on strips $] 0, T\left[\times \mathbb{R}^{n}\right.$ and there, the main part of the work is to prove good energy estimates for (weak) solutions. In this framework, the results of Theorem 1.5 are improved by using non isotropic spaces, and by making a detailed account of the loss of spatial smoothness as time evolves, as in [5, 6]. The precise results are stated in section 2 below and are proved in section 4 using the paradifferential calculus of J.-M. Bony, whose LL-version is presented in section 3.

\section{The global in space problem}

In this section we denote by $(t, x)$ the space-time variables. On $\Omega=\left[0, T_{0}\right] \times \mathbb{R}^{n}$ consider a second order hyperbolic differential operator

$$
L u=L_{2} u+L_{1} u+d u
$$

with

$$
\begin{aligned}
& L_{2}=\partial_{t} a_{0} \partial_{t}+\sum_{j=1}^{n}\left(\partial_{t} a_{j} \partial_{x_{j}}+\partial_{x_{j}} a_{j} \partial_{t}\right)-\sum_{j, k=1}^{n} \partial_{x_{j}} a_{j, k} \partial_{x_{k}}, \\
& L_{1}=b_{0} \partial_{t}+\partial_{t} c_{0}+\sum_{j=1}^{n}\left(b_{j} \partial_{x_{j}}+\partial_{x_{j}} c_{j}\right) .
\end{aligned}
$$

The coefficients satisfy on $\Omega=\left[0, T_{0}\right] \times \mathbb{R}^{n}$

$$
\begin{gathered}
a_{j, k}=a_{k, j}, \quad a_{0}, a_{j}, a_{j, k} \in L^{\infty}(\Omega) \cap L L(\Omega), \\
b_{0}, c_{0}, b_{j}, c_{j} \in L^{\infty}(\Omega) \cap C^{\alpha}(\Omega), \\
d \in L^{\infty}(\Omega),
\end{gathered}
$$

for some $\alpha \in] \frac{1}{2}, 1\left[\right.$. Recall that the space LL is defined by (1.3), the semi-norm $\|a\|_{L L}$ being the best constant $C$ in (1.3). In addition, for $\alpha \in] 0,1\left[, C^{\alpha}\right.$ denotes the usual Hölder space, equipped with the norm

$$
\|a\|_{C^{\alpha}}=\|a\|_{L^{\infty}}+\sup _{y \neq y^{\prime}} \frac{\left|a(y)-a\left(y^{\prime}\right)\right|}{\left|y-y^{\prime}\right|^{\alpha}} .
$$

When $\alpha=1$, this defines the norm $\|a\|_{L i p}$ in the space of Lipschitz functions. 
We assume that $L$ is hyperbolic in the direction $d t$, which means that there are $\delta_{0}>0$ and $\delta_{1}>0$ such that for all $(t, x, \xi) \in\left[0, T_{0}\right] \times \mathbb{R}^{n} \times \mathbb{R}^{n}$

$$
a_{0}(t, x) \geq \delta_{0}, \quad \sum_{1 \leq j, k \leq n}\left(a_{j, k}+\frac{a_{j} a_{k}}{a_{0}}\right) \xi_{j} \xi_{k} \geq \delta_{1}|\xi|^{2} .
$$

We denote by $A_{L^{\infty}}, A_{L L}$ and $B$ constants such that for all indices

$$
\begin{aligned}
\left\|a_{0}, a_{j}, a_{j, k}\right\|_{L^{\infty}(\Omega)} & \leq A_{L^{\infty}}, & \left\|a_{0}, a_{j}, a_{j, k}\right\|_{L L(\Omega)} & \leq A_{L L}, \\
\left\|b_{0}, c_{0}, b_{j}, c_{j}\right\|_{C^{\alpha}(\Omega)} & \leq B, & \|d\|_{L^{\infty}(\Omega)} & \leq B .
\end{aligned}
$$

\subsection{Giving sense to the Cauchy problem}

Consider the vector fields

$$
X=a_{0} \partial_{t}+\sum_{j=1}^{n} a_{j} \partial_{x_{j}}=a_{0} Y .
$$

Formal computations immediately show that the second order part of $L$ can be written

$$
L_{2} u=Z X u-\tilde{L}_{2} u
$$

with

$$
Z v=\partial_{t} v+\sum_{j=1}^{n} \partial_{x_{j}}\left(\tilde{a}_{j} v\right), \quad \tilde{L}_{2} u=\sum_{j, k=1}^{n} \partial_{x_{j}}\left(\tilde{a}_{j, k} \partial_{x_{k}} u\right),
$$

$\tilde{a}_{j, k}=a_{j, k}+a_{j} a_{k} / a_{0}$, and $\tilde{a}_{j}=a_{j} / a_{0}$. Consequently, it follows that

$$
L u=\left(Z+\tilde{b}_{0}\right)\left(X+c_{0}\right) u-\tilde{L}_{2} u+\tilde{L}_{1} u+\tilde{d} u
$$

with

$$
\tilde{L}_{1} u=\sum_{j=1}^{n} \tilde{b}_{j} \partial_{x_{j}} u+\sum_{j=1}^{n} \partial_{x_{j}}\left(\tilde{c}_{j} u\right)
$$

and

$$
\tilde{b}_{0}=b_{0} / a_{0}, \quad \tilde{b}_{j}=b_{j}-\tilde{b}_{0} a_{j}, \quad \tilde{c}_{j}=c_{j}-\tilde{a}_{j} c_{0}, \quad \tilde{d}=d-c_{0} \tilde{c}_{0} .
$$

The next lemma shows that these identities are rigorous under minimal smoothness assumption on $u$.

Lemma 2.1. - Suppose that $u \in H^{\rho}(] 0, T\left[\times \mathbb{R}^{n}\right)$ for some $\left.\rho \in\right] 1-\alpha, \alpha[$. Then $\mathrm{cu \text {, }}$ $X u$ and $L_{1} u$ belong to $H^{\rho-1}(] 0, T\left[\times \mathbb{R}^{n}\right)$. Moreover $L_{2} u$ is well defined as a distribution in $H^{\rho-2}(] 0, T\left[\times \mathbb{R}^{n}\right)$.

Proof. - Both $u$ and its space-time derivatives $\left(\partial_{t} u, \partial_{x_{j}} u\right)$ belong to $H^{\rho-1}$. Following [6], their multiplication by a bounded LL function belong to the same space (see also Corollary 3.6). This shows that all the individual terms present in the definition of $X u$ belong to $H^{\rho-1}$ and those occurring in $L_{2} u$ and $Z X u$ are well defined in $H^{\rho-2}$ in the sense of distributions.

Next we recall that the multiplication $(b, u) \mapsto b u$ is continuous from $C^{\alpha} \times H^{s}$ to $H^{s}$ when $|s|<\alpha$. This implies that the terms $b \partial u$ and $\partial(c u)$ that occur in $L_{1} u$ and $\tilde{L}_{1} u$ belong to $H^{\rho-1}$ since $\left.\rho \in\right] 1-\alpha, \alpha[$.

The last term $d u$ is in $L^{2}$, thus in $H^{\rho-1}$, since $c \in L^{\infty}$ and $u \in L^{2}$. 
The identity (2.12) is straightforward from (2.2) since all the algebraic computations make sense by the preceding remarks.

Next we need partial regularity results in time, showing that the traces of $u$ and $X u$ at $t=0$ are well defined, as distributions, for solutions of $L u=f$. This is based on the remark that this equation is equivalent to the system

$$
\left\{\begin{array}{l}
Z v+\tilde{b}_{0} v=\tilde{L}_{2} u-\tilde{L}_{1} u-\tilde{d} u+f \\
Y u+\tilde{c}_{0} u=v / a_{0}
\end{array}\right.
$$

with $\tilde{c}_{0}=c_{0} / a_{0}$. The important remark is that, for this system, the coefficients of $\partial_{t}$, both for $u$ and $v$, are equal to 1 , thus smooth. Using the notation $Y=\partial_{t}+\widetilde{Y}, Z=\partial_{t}+\widetilde{Z}$, the system reads

$$
\left\{\begin{array}{l}
\partial_{t} v=-\tilde{Z} v-\tilde{b}_{0} v-\tilde{L}_{2} u-\tilde{L}_{1} u-\tilde{d} u+f \\
\partial_{t} u=-\tilde{Y} u+v / a_{0} .
\end{array}\right.
$$

Lemma 2.2. - Suppose that $\rho \in] 1-\alpha, \alpha\left[\right.$ and $u \in H^{\rho}(] 0, T\left[\times \mathbb{R}^{n}\right)$ is such that Lu $\in$ $L^{1}\left([0, T] ; H^{\rho-1}\left(\mathbb{R}^{n}\right)\right)$. Then $u \in L^{2}\left([0, T] ; H^{\rho}\left(\mathbb{R}^{n}\right)\right)$ and $\partial_{t} u \in L^{2}\left([0, T] ; H^{\rho-1}\left(\mathbb{R}^{n}\right)\right)$. Therefore, $u \in C^{0}\left([0, T] ; H^{\rho-\frac{1}{2}}\left(\mathbb{R}^{n}\right)\right)$.

Moreover, $X u \in L^{2}\left([0, T] ; H^{\rho-1}\left(\mathbb{R}^{n}\right)\right)$ and $X u \in C^{0}\left([0, T] ; H^{\rho-\frac{3}{2}}\left(\mathbb{R}^{n}\right)\right)$.

In particular, the traces $u_{\mid t=0}$ and $X u_{\mid t=0}$ are well defined in $H^{\rho-\frac{1}{2}}\left(\mathbb{R}^{n}\right)$ and $H^{\rho-\frac{3}{2}}\left(\mathbb{R}^{n}\right)$, respectively.

Proof. - a) We use the spaces $H^{s, s^{\prime}}$ of Hörmander ([7], chapter 2), which are defined on $\mathbb{R}^{1+n}$ as the spaces of temperate distributions such that their Fourier transform $\hat{u}$ satisfies $\left(1+\tau^{2}+|\xi|^{2}\right)^{s / 2}\left(1+|\xi|^{2}\right)^{s^{\prime} / 2} \hat{u} \in L^{2}$. The spaces on $[0, T] \times \mathbb{R}^{n}$ are defined by restriction. In particular, $H^{0, s^{\prime}}\left([0, T] \times \mathbb{R}^{n}\right)=L^{2}\left([0, T] ; H^{s^{\prime}}\left(\mathbb{R}^{n}\right)\right)$. Recall that $\partial_{x_{j}}$ maps $H^{s, s^{\prime}}$ to $H^{s, s^{\prime}-1}$ and that

$$
u \in H^{s, s^{\prime}}, \partial_{t} u \in H^{s, s^{\prime}-1} \Rightarrow u \in H^{s+1, s^{\prime}-1} .
$$

b) For $u \in H^{\rho}$, the first derivatives of $u, \tilde{d} u$, as well as $\tilde{L}_{1} u, X u$ and $v$ belong to $H^{\rho-1}=$ $H^{\rho-1,0}$, as well as their multiplication by a LL or $C^{\alpha}$ coefficient. Thus $\tilde{L}_{2} u$ and $\tilde{Z} v$ belong to $H^{\rho-1,-1}$ and

$$
\partial_{t} v=f+g, \quad f=L u \in L^{1}(] 0, T\left[; H^{\rho-1}\right), g \in H^{\rho-1,-1} .
$$

Let

$$
v_{0}(t)=\int_{0}^{t} f\left(t^{\prime}\right) d t^{\prime} \in C^{0}\left(H^{\rho-1}\right) .
$$

In particular, $v \in L^{2}(] 0, T\left[; H^{\rho-1}\right)=H^{0, \rho-1} \subset H^{\rho-1,0}$, since $\rho-1 \leq 0$. Thus, $v-v_{0} \in$ $H^{\rho-1,0}$ and $\partial_{t}\left(v-v_{0}\right)=g \in H^{\rho-1,-1}$. By (2.18) $v-v_{0} \in H^{\rho,-1} \subset H^{0, \rho-1}$ since $\rho \geq 0$.

Next, reasoning for fixed time and then taking $L^{2}$ norms we note that the multiplication by a $\mathrm{LL}$ or $C^{\alpha}$ function maps $L^{2}(] 0, T\left[; H^{\rho-1}\right)=H^{0, \rho-1}$ into itself. Thus, by the second equation of (2.17), $\partial_{t} u=-\tilde{Y} u+v / a_{0} \in H^{0, \rho-1}$. This finishes the proof of the first part of the lemma.

c) In particular, it implies that $v=X u+b_{0} u \in H^{0, \rho-1}$. Thus, $\tilde{Z} v$ and $\tilde{L}_{2} u$ which involve multiplication by $C^{\alpha}$ or LL function, followed by a spatial derivative, belong to $H^{0, \rho-2}$. Therefore, the equation implies that in (2.19) $g \in H^{0, \rho-2}$. Thus applying (2.18) to $v-v_{0} \in$ 
$H^{0, \rho-1}$ implies that $v-v_{0} \in H^{1, \rho-2} \subset C^{0}\left([0, T] ; H^{\rho-\frac{3}{2}}\left(\mathbb{R}^{n}\right)\right)$. Since $\left|\rho-\frac{1}{2}\right|<\alpha$ and $u \in C^{0}\left([0, T] ; H^{\rho-\frac{1}{2}}\left(\mathbb{R}^{n}\right)\right)$, the product $\tilde{b}_{0} u$ belongs to $C^{0}\left([0, T] ; H^{\rho-\frac{1}{2}}\left(\mathbb{R}^{n}\right)\right)$. Since $v_{0}$ is also in this space, we conclude that $X u \in C^{0}\left([0, T] ; H^{\rho-\frac{3}{2}}\left(\mathbb{R}^{n}\right)\right)$.

REMARK 2.3. - If $\rho>\frac{1}{2}$, then the multiplication by LL functions maps $H^{\rho-\frac{3}{2}}$ into itself and we can conclude that $\partial_{t} u \in C^{0}\left([0, T] ; H^{\rho-\frac{3}{2}}\left(\mathbb{R}^{n}\right)\right)$, as well as all the first derivatives of $u$, so that their traces at $t=0$ are well defined. When $\rho \leq \frac{1}{2}$, the continuity of $\partial_{t} u$ is not clear. However, the trace of $X u$ has an intrinsic meaning, as a consequence of Proposition 1.4 (see Section 5).

Lemma 2.2 allows us to consider the Cauchy problem

$$
L u=f, \quad u_{\mid t=0}=u_{0}, \quad X u_{\mid t=0}=u_{1},
$$

when $f \in \bigcup_{\rho>-\alpha} L^{1}\left([0, T] ; H^{\rho}\left(\mathbb{R}^{n}\right)\right)$ and $u \in \bigcup_{\rho>1-\alpha} H^{\rho}(] 0, T\left[\times \mathbb{R}^{n}\right)$.

\subsection{The main results}

We first state uniqueness for the Cauchy problem:

TheOREM 2.4. - If $u \in \bigcup_{\rho>1-\alpha} H^{\rho}(] 0, T\left[\times \mathbb{R}^{n}\right)$ satisfies

$$
L u=0, \quad u_{\mid t=0}=0, \quad X u_{\mid t=0}=0
$$

then $u=0$.

As in [5, 6], we prove existence of solutions in Sobolev spaces having orders decreasing in time. The proper definition is given as follows. The operators

$$
|D| \quad \text { and } \quad \Lambda:=\log (2+|D|)
$$

are defined by Fourier transform, associated to the Fourier multipliers $|\xi|$ and $\log (2+|\xi|)$ respectively.

Definition 2.5. - $\quad$ i) $H^{s}\left(\mathbb{R}^{n}\right)$ or $H^{s}$ denotes the usual Sobolev space on $\mathbb{R}^{n} . H^{s+\frac{1}{2} \log }$ and $H^{s-\frac{1}{2} \log }$ denote the spaces $\Lambda^{-\frac{1}{2}} H^{s}$ and $\Lambda^{\frac{1}{2}} H^{s}$ respectively.

ii) Given parameters $\sigma$ and $\lambda$, we denote by $\mathcal{C}_{\sigma, \lambda}(T)$ the space of functions u such that for all $t_{0} \in[0, T], u \in C^{0}\left(\left[0, t_{0}\right], H^{\sigma-\lambda t_{0}}\right)$.

iii) $\mathcal{H}_{\sigma \pm \frac{1}{2} \log , \lambda}(T)$ denotes the spaces of functions $u$ on $[0, T]$ with values in the space of temperate distributions in $\mathbb{R}^{n}$ such that

$$
(1+|D|)^{\sigma-\lambda t} \Lambda^{ \pm \frac{1}{2}} u(t, \cdot) \in L^{2}\left([0, T] ; L^{2}\left(\mathbb{R}^{n}\right)\right) .
$$

iv) $\mathcal{L}_{\sigma, \lambda}(T)$ denotes the space of functions $u$ on $[0, T]$ with values in the space of temperate distributions in $\mathbb{R}^{n}$ such that

$$
(1+|D|)^{\sigma-\lambda t} u(t, \cdot) \in L^{1}\left([0, T] ; L^{2}\left(\mathbb{R}^{n}\right)\right) .
$$


$\mathcal{C}_{\sigma, \lambda}(T)$ is equipped with the norm

$$
\sup _{t \in[0, T]}\|u(t)\|_{H^{\sigma-\lambda t}} .
$$

The norms in $\mathcal{H}_{\sigma \pm \frac{1}{2} \log , \lambda}(T)$ and $\mathcal{L}_{\sigma, \lambda}(T)$ are given by (2.23) and (2.24). Equivalently, $\mathcal{H}_{\sigma \pm \frac{1}{2} \log , \lambda}(T)$ and $\mathcal{L}_{\sigma, \lambda}(T)$ are the completions of $C_{0}^{\infty}\left([0, T] \times \mathbb{R}^{n}\right)$ for the norms

$$
\|u\|_{\mathcal{H}_{\sigma \pm \frac{1}{2} \log , \lambda}(T)}=\left(\int_{0}^{T}\|u(t)\|_{H^{\sigma-\lambda t \pm \frac{1}{2} \log }}^{2} d t\right)^{\frac{1}{2}} .
$$

and

$$
\|u\|_{\mathcal{L}_{\sigma, \lambda}(T)}=\int_{0}^{T}\|u(t)\|_{H^{\sigma-\lambda t}} d t .
$$

Theorem 2.6. - Fix $\theta<\theta_{1}$ in $] 1-\alpha, \alpha[$. Then there are $\lambda>0$ and $K>0$, which depend only on the constants $A_{L^{\infty}}, A_{L L}, B, \delta_{0}, \delta_{1}, \theta$ and $\theta_{1}$, given by (2.8), (2.9) and (2.10), such that for

$$
T=\min \left\{T_{0}, \frac{\theta_{1}-\theta}{\lambda}\right\}
$$

$u_{0} \in H^{1-\theta}\left(\mathbb{R}^{n}\right), u_{1} \in H^{-\theta}\left(\mathbb{R}^{n}\right)$ and $f=f_{1}+f_{2}$ with $f_{1} \in \mathcal{L}_{-\theta, \lambda}(T)$ and $f_{2} \in \mathcal{H}_{-\theta-\frac{1}{2} \log , \lambda}(T)$, the Cauchy problem (2.20), has a unique solution $u \in \mathcal{C}_{1-\theta, \lambda}(T) \cap \mathcal{H}_{1-\theta+\frac{1}{2} \log , \lambda}(T)$ with $\partial_{t} u \in$ $\mathcal{C}_{-\theta, \lambda}(T) \cap \mathcal{H}_{-\theta+\frac{1}{2} \log , \lambda}(T)$. Moreover, it satisfies

$$
\begin{aligned}
& \sup _{0 \leq t^{\prime} \leq t}\left\|u\left(t^{\prime}\right)\right\|_{H^{1-\theta-\lambda t^{\prime}}}^{2}+\sup _{0 \leq t^{\prime} \leq t}\left\|\partial_{t} u\left(t^{\prime}\right)\right\|_{H^{-\theta-\lambda t^{\prime}}}^{2} \\
& \quad+\int_{0}^{t}\left(\left\|u\left(t^{\prime}\right)\right\|_{H^{1-\theta-\lambda t^{\prime}+\frac{1}{2} \log }}^{2}+\left\|\partial_{t} u\left(t^{\prime}\right)\right\|_{H^{-\theta-\lambda t^{\prime}+\frac{1}{2} \log }}^{2}\right) d t^{\prime}
\end{aligned}
$$

$$
\begin{aligned}
\leq & K\left\{\left\|u_{0}\right\|_{H^{1-\theta}}^{2}+\left\|u_{1}\right\|_{H^{-\theta}}^{2}\right. \\
& \left.+\left(\int_{0}^{t}\left\|f_{1}\left(t^{\prime}\right)\right\|_{H^{-\theta-\lambda t^{\prime}}} d t^{\prime}\right)^{2}+\int_{0}^{t}\left\|f_{2}\left(t^{\prime}\right)\right\|_{H^{-\theta-\lambda t^{\prime}-\frac{1}{2} \log }}^{2} d t^{\prime}\right\} .
\end{aligned}
$$

Note that for $t \in[0, T], 1-\theta-\lambda t \geq 1-\theta_{1}>1-\alpha$, so that $f \in L^{1}\left([0, T] ; H^{-\theta_{2}}\right)$ with $\theta_{1}<\theta_{2}<\alpha$. Similarly, $u \in L^{2}\left([0, T] ; H^{1-\theta_{1}}\right)$ and $\partial_{t} u \in L^{2}\left([0, T] ; H^{-\theta_{1}}\right)$ implying that $u \in H^{1-\theta_{1}}\left([0, T] \times \mathbb{R}^{n}\right)$. Therefore, we are in a situation where we have given sense to the Cauchy problem.

REMARK 2.7. - This is a local in time existence theorem since the life span (2.28) is limited by the choice of $\lambda$. Thus the dependence of $\lambda_{0}$ on the coefficient is of crucial importance. In case of Lipschitz coefficients, there is no loss of derivatives; this would correspond to $\lambda=0$. Using the notations in (2.9) (2.10) and (2.8), the analysis of the proof below shows that there is a function $K_{0}(\cdot)$ such that one can choose

$$
\lambda=\frac{A_{L L}}{\min \left\{\delta_{0}, \delta_{1}\right\}} K_{0}\left(\frac{A_{L^{\infty}}}{\delta_{0}}\right),
$$

revealing the importance of the LL-norms of the coefficients and the role of the hyperbolicity constant $\delta_{1} / \delta_{0}$. In particular, it depends only on the second order part of operator $L$. 
REMARK 2.8. - A closer inspection of the proof also shows that if the coefficients of the principal part of $L$ are $\left(a_{0}, a_{j}, a_{j, k}\right)=\left(a_{0}^{\prime}+a_{0}^{\prime \prime}, a_{j}^{\prime}+a_{j}^{\prime \prime}, a_{j, k}^{\prime}+a_{j, k}^{\prime \prime}\right)$ with $\left(a_{0}^{\prime}, a_{j}^{\prime}, a_{j, k}^{\prime}\right)$ Lipschitz continous and $\left(a_{0}^{\prime \prime}, a_{j}^{\prime \prime}, a_{j, k}^{\prime \prime}\right) \log$ Lipschitz, with LL norm bounded by $A_{L L}^{\prime \prime}$, one can replace $A_{L L}$ by $A_{L L}^{\prime \prime}$ in the definition of $\lambda$. In particular if instead of (1.3) the coefficients satisfy

$$
\left|a(y)-a\left(y^{\prime}\right)\right| \leq C \omega\left(\left|y-y^{\prime}\right|\right)
$$

with a modulus of continuity $\omega$ such that

$$
\lim _{\varepsilon \rightarrow 0+} \frac{\omega(\varepsilon)}{\varepsilon|\log \varepsilon|}=0
$$

they can be approximated by Lipschitz functions with errors arbitrarily small in the LL norm. This can be done by usual mollifications, which will preserve the $L^{\infty}$ bounds $A_{L^{\infty}}$ and keep uniform hyperbolicity constants $\delta_{0}$ and $\delta_{1}$. As a consequence, $\lambda$ can be taken arbitrarily small, yielding global in time existence with arbitrarily small loss of regularity (see Theorem 2.1 in [3] when the coefficients depend only on time).

\section{Paradifferential calculus with LL coefficients}

In this section we review several known results on paradifferential calculus and give the needed extensions to the case of Log-Lipschitz coefficients.

\subsection{The Paley-Littlewood analysis}

Introduce $\chi \in C_{0}^{\infty}(\mathbb{R})$, real valued, even and such that $0 \leq \chi \leq 1$ and

$$
\chi(\xi)=1 \quad \text { for }|\xi| \leq 1.1, \quad \chi(\xi)=0 \text { for }|\xi| \geq 1.9 .
$$

For $k \in \mathbb{Z}$, introduce $\chi_{k}(\xi):=\chi\left(2^{-k} \xi\right), \tilde{\chi}_{k}(x)$ its inverse Fourier transform with respect to $\xi$ and the operators

$$
\begin{gathered}
S_{k} u:=\widetilde{\chi}_{k} * u=\chi_{k}\left(D_{x}\right) u, \\
\Delta_{0}=S_{0}, \quad \text { and for } k \geq 1 \quad \Delta_{k}=S_{k}-S_{k-1} .
\end{gathered}
$$

We note that $\Delta_{k}$ and $S_{k}$ are self adjoint. Moreover, by evenness, $\widetilde{\chi}_{k}$ is real, so that $\Delta_{k}$ and $S_{k}$ preserve reality. For all temperate distributions $u$ one has

$$
u=\sum_{k \geq 0} \Delta_{k} u \text {. }
$$

The next propositions immediately follow from the definitions.

Proposition 3.1. - Consider $s \in \mathbb{R}$. A temperate distribution u belongs to $H^{s}\left(\mathbb{R}^{n}\right)$ (resp. $H^{s \pm \frac{1}{2} \log }$ ) if and only if

i) for all $k \in \mathbb{N}, \Delta_{k} u \in L^{2}\left(\mathbb{R}^{d}\right)$.

ii) the sequence $\delta_{k}=2^{k s}\left\|\Delta_{k} u\right\|_{L^{2}\left(\mathbb{R}^{d}\right)}$ (resp. $\delta_{k}=(k+1)^{ \pm \frac{1}{2}} 2^{k s}\left\|\Delta_{k} u\right\|_{L^{2}\left(\mathbb{R}^{d}\right)}$ ) belongs to $\ell^{2}(\mathbb{N})$.

Moreover, the norm of the sequence $\delta_{k}$ in $\ell^{2}$ is equivalent to the norm of $u$ in the given space.

Proposition 3.2. - Consider $s \in \mathbb{R}$ and $R>0$. Suppose that $\left\{u_{k}\right\}_{k \in \mathbb{N}}$ is a sequence of functions in $L^{2}\left(\mathbb{R}^{d}\right)$ such that:

$4^{\mathrm{e}}$ SÉRIE - TOME $41-2008-\mathrm{N}^{\mathrm{o}} 2$ 
i) the spectrum of $u_{0}$ is contained in $\{|\xi| \leq R\}$ and for $k \geq 1$ the spectrum of $u_{k}$ is contained in $\left\{\frac{1}{R} 2^{k} \leq|\xi| \leq R 2^{k}\right\}$.

ii) the sequence $\delta_{k}=2^{k s}\left\|u_{k}\right\|_{L^{2}\left(\mathbb{R}^{d}\right)}$ (resp. $\delta_{k}=(k+1)^{ \pm \frac{1}{2}} 2^{k s}\left\|\Delta_{k} u\right\|_{L^{2}\left(\mathbb{R}^{d}\right)}$ ) belongs to $\ell^{2}(\mathbb{N})$.

Then $u=\sum u_{k}$ belongs to $H^{s}\left(\mathbb{R}^{d}\right)$ (resp. $\left.H^{s \pm \frac{1}{2} \log }\right)$. Moreover, the norm of the sequence $\delta_{k}$ in $\ell^{2}$ is equivalent to the norm of $u$ in the given space.

When $s>0$, it is sufficient to assume that the spectrum of $u_{k}$ is contained in $\left\{|\xi| \leq R 2^{k}\right\}$.

Next we collect several results about the dyadic analysis of LL spaces.

Proposition 3.3. - There is a constant $C$ such that for all $a \in L L\left(\mathbb{R}^{n}\right)$ and all integers $k>0$

$$
\left\|\Delta_{k} a\right\|_{L^{\infty}} \leq C k 2^{-k}\|a\|_{L L}
$$

Moreover, for all $k \geq 0$

$$
\begin{aligned}
\left\|a-S_{k} a\right\|_{L^{\infty}} & \leq C(k+1)\|a\|_{L L} \\
\left\|S_{k} a\right\|_{L i p} & \leq C\left(\|a\|_{L^{\infty}}+(k+1)\|a\|_{L L}\right) .
\end{aligned}
$$

If $\alpha \in] 0,1\left[\right.$ and $a \in C^{\alpha}\left(\mathbb{R}^{n}\right)$, then

$$
\left\|\Delta_{k} a\right\|_{L^{\infty}} \leq C 2^{-\alpha k}\|a\|_{C^{\alpha}} .
$$

Proof. $-S_{k}$ is a convolution operator with $\tilde{\chi}_{k}$ which is uniformly bounded in $L^{1}$. Thus

$$
\left\|S_{k} a\right\|_{L^{\infty}} \leq C\|a\|_{L^{\infty}} \text {. }
$$

Moreover, since the integral of $\partial_{j} \tilde{\chi}_{k}$ vanishes

$$
\partial_{j}\left(S_{k} a\right)(x)=\int \partial_{j} \tilde{\chi}_{k}(y)(a(x-y)-a(x)) d y .
$$

Using the LL smoothness of $a$ yields

$$
\left\|\nabla S_{k} a\right\|_{L^{\infty}} \leq C(k+1)\|a\|_{L L} .
$$

This implies (3.6). The proof of (3.4) is similar (cf [6]). The third estimate is classical.

\subsection{Paraproducts}

Following J.-M. Bony ([2]), for $N \geq 3$ one defines the para-product of $a$ and $u$ as

$$
T_{a}^{N} u=\sum_{k=N}^{\infty} S_{k-N} a \Delta_{k} u
$$

The remainder $R_{a}^{N} u$ is defined as

$$
R_{a}^{N} u=a u-T_{a}^{N} u
$$

The next proposition extends classical results (see $[2,13])$ to the case of LL coefficients and Log Sobolev spaces.

Proposition 3.4. - $\quad$ i) For $a \in L^{\infty}$ and $s \in \mathbb{R}$, $T_{a}^{N}$ continuously maps $H^{s}$ to $H^{s}$ and $H^{s \pm \frac{1}{2} \log }$ to $H^{s \pm \frac{1}{2} \log }$. Moreover, the operator norms are uniformly bounded for $s$ in a compact set. 
ii) If $a \in L^{\infty} \cap L L$ and $N^{\prime} \geq N \geq 3, T_{a}^{N}-T_{a}^{N^{\prime}}$ maps $H^{s+\frac{1}{2} \log }$ into $H^{s+1-\frac{1}{2} \log }$, for all $s \in \mathbb{R}$.

iii) If $a \in L^{\infty} \cap L L, N \geq 3$ and $\left.s \in\right] 0,1\left[, R_{a}^{N}\right.$ maps $H^{-s+\frac{1}{2} \log }$ into $H^{1-s-\frac{1}{2} \log }$, and

$$
\left\|R_{a}^{N} u\right\|_{H^{1-s-\frac{1}{2} \log }} \leq C\|a\|_{L L}\|u\|_{H^{-s+\frac{1}{2} \log }}
$$

with $C$ uniformly bounded for $s$ in a compact subset of $] 0,1[$.

Proof. - The first statement is an immediate consequence of (3.8) and Propositions 3.1 and 3.2 .

Next, $T_{a}^{N} u-T_{a}^{N^{\prime}} u=\sum_{k} v_{k}$ with $v_{k}=\left(S_{k-N} a-S_{k-N^{\prime}} a\right) \Delta_{k} u$. By Proposition 3.3

$$
\left\|v_{k}\right\|_{L^{2}} \leq C(k+1) 2^{-k}\left\|\Delta_{k} u\right\|_{L^{2}} .
$$

With Proposition 3.2, this implies ii).

To prove iii) we can assume that $N=3$. Then

$$
R_{a} u=\sum_{k \geq 3} \Delta_{k} a S_{k-3} u+\sum_{k} \sum_{|k-j| \leq 2} \Delta_{j} a \Delta_{k} u .
$$

If $u \in H^{-s+\frac{1}{2} \log }$, then

$$
\left\|\Delta_{j} u\right\|_{L^{2}} \leq \frac{2^{j s}}{\sqrt{j+1}} \varepsilon_{j}
$$

with $\left\{\varepsilon_{j}\right\} \in \ell^{2}$. We note that the sequence

$$
\widetilde{\varepsilon}_{k}=\sum_{j \leq k} \frac{\sqrt{k+1}}{\sqrt{j+1}} 2^{(j-k) s} \varepsilon_{j}
$$

is also in $\ell^{2}$ with

$$
\left\|\widetilde{\varepsilon}_{k}\right\|_{\ell^{2}} \leq C\left\|\varepsilon_{j}\right\|_{\ell^{2}}
$$

with $C$ uniformly bounded when $s$ in a compact subset of $] 0,+\infty]$. Thus

$$
\left\|S_{k-3} u\right\|_{L^{2}} \leq \frac{2^{k s}}{\sqrt{k+1}} \varepsilon_{k}^{\prime}
$$

with $\left\{\varepsilon_{k}^{\prime}\right\} \in \ell^{2}$. Therefore,

$$
\left\|\Delta_{k} a S_{k-3} u\right\|_{L^{2}} \leq C \sqrt{k+1} 2^{(s-1) k} \varepsilon_{k}^{\prime} .
$$

Proposition 3.2 implies that the first sum in (3.13) belongs to $H^{1-s-\frac{1}{2} \log }$.

Similarly,

$$
\left\|\sum_{|k-j| \leq 2} \Delta_{j} a \Delta_{k} u\right\|_{L^{2}} \leq C \sqrt{k+1} 2^{(s-1) k} \varepsilon_{k}^{\prime \prime},
$$

with $\left\{\varepsilon_{k}^{\prime \prime}\right\} \in \ell^{2}$. Now the spectrum of $\Delta_{j} a \Delta_{k} u$ is contained in the ball $\left\{|\xi| \leq 2^{k+3}\right\}$; because

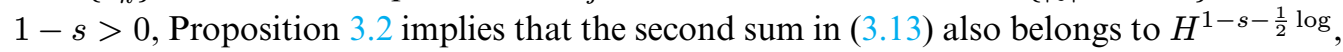
and the norm is uniformly bounded when $s$ remains in a compact subset of $[0,1[$.

REMARK 3.5. - By ii) we see that the choice of $N \geq 3$ is essentially irrelevant in our analysis, as in [2]. To simplify notation, we make a definite choice of $N$, for instance $N=3$, and use the notation $T_{a}$ and $R_{a}$ for $T_{a}^{N}$ and $R_{a}^{N}$. 
COROLlary 3.6. - The multiplication $(a, u) \mapsto$ au is continuous from $\left(L^{\infty} \cap L L\right) \times$ $H^{s+\delta \log }$ to $H^{s+\delta \log }$ for $\left.s \in\right]-1,1\left[\right.$ and $\delta \in\left\{-\frac{1}{2}, 0, \frac{1}{2}\right\}$.

Proof. - (See [6].) Property iii) says that $R_{a}$ is smoothing by almost one derivative in negative spaces, and therefore, for all $\sigma \in]-1,1\left[\right.$ it maps $H^{\sigma}$ to $H^{\sigma^{\prime}}$ for all $\sigma^{\prime}>\max \{\sigma, 0\}$ such that $\sigma^{\prime}<\min \{\sigma+1,1\}$. Combining this observation with i), the corollary follows.

In particular, we note the following estimate

$$
\|a u\|_{H^{s+\frac{1}{2} \log }} \leq C\left(\|a\|_{L^{\infty}}\|u\|_{H^{s+\frac{1}{2} \log }}+\|a\|_{L L}\|u\|_{H^{s}}\right) .
$$

Proposition 3.7. - Consider $q=\sqrt{\left(1+|\xi|^{2}\right)}$ and $\psi(\xi)$ a symbol of degree $m$ on $\mathbb{R}^{n}$. Denote by $Q=\sqrt{(1-\Delta)}$ and $\Psi$ the associated operators. If $a \in L^{\infty} \cap L L$, then the commutator $\left[Q^{-s} \Psi, T_{a}\right]$ maps $H^{-s+\frac{1}{2} \log }$ into $H^{1-m-\frac{1}{2} \log }$ and

$$
\left\|\left[Q^{-s} \Psi, T_{a}\right] u\right\|_{H^{1-m-\frac{1}{2} \log }} \leq C\|a\|_{L L}\|u\|_{H^{-s+\frac{1}{2} \log }}
$$

with $C$ uniformly bounded for $s \in[0,1]$ and $\psi$ in a bounded set.

Proof. - We use Theorem 35 of [4], which states that if $H$ is a Fourier multiplier with symbol $h$ of degree 0 and if $a$ is Lipschitz, then

$$
\left\|[H, a] \partial_{x_{j}} u\right\|_{L^{2}} \leq C\left\|\nabla_{x} a\right\|_{L^{\infty}}\|u\|_{L^{2}} .
$$

For $k>0$, writing $\Delta_{k} u$ as sum of derivatives, this implies that

$$
\left\|[H, a] \Delta_{k} u\right\|_{L^{2}} \leq C 2^{-k}\left\|\nabla_{x} a\right\|_{L^{\infty}}\left\|\Delta_{k} u\right\|_{L^{2}},
$$

with $C$ independent of $k$ and $H$, provided that the symbol $h$ remains in a bounded set of symbols of degree 0 .

We now proceed to the proof of the proposition. Since $\Psi$ and $Q$ commute with $\Delta_{k}$, one has

$$
\left[Q^{-s} \Psi, T_{a}\right] u=\sum_{k \geq 3}\left[Q^{-s} \Psi, S_{k-3} a\right] \Delta_{k} u .
$$

Moreover, since the spectrum of $S_{k-3} a \Delta_{k} u$ is contained in the annulus $2^{k-1} \leq|\xi| \leq 2^{k+2}$, it follows that

$$
\left[Q^{-s} \Psi, S_{k-3} a\right] \Delta_{k}=2^{k(m-s)}\left[H_{k}, S_{k-3} a\right] \Delta_{k}
$$

where the symbol of $H_{k}$ is

$$
h_{k}(\xi)=2^{k(s-m)} q^{-s}(\xi) \psi(\xi) \varphi\left(2^{-k} \xi\right)
$$

and $\varphi$ supported in a suitable fixed annulus. Note that the family $\left\{h_{k}\right\}$ is bounded in the space of symbols of degree 0 , uniformly in $k, s \in[0,1]$ and $\psi$ in a bounded set of symbols of degree $m$. By (3.17), it follows that

$$
\left\|\left[H_{k}, S_{k-3} a\right] \Delta_{k} u\right\|_{L^{2}} \leq C 2^{k(m-s-1)}\left\|\nabla S_{k-3} a\right\|_{L^{\infty}}\left\|\Delta_{k} u\right\|_{L^{2}} .
$$

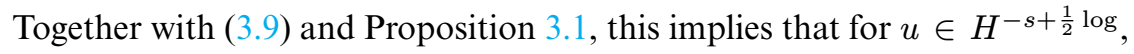

$$
\left\|\left[Q^{-s} \Psi, S_{k-3} a\right] \Delta_{k} u\right\| \leq C(k+1)\|a\|_{L L}\left\|\Delta_{k} u\right\|_{L^{2}} .
$$

Using Proposition 3.2, the estimate (3.16) follows. 
Proposition 3.8. - If $a \in L^{\infty} \cap L L$ is real valued, then $\left(T_{a}-\left(T_{a}\right)^{*}\right) \partial_{x_{j}}$ and $\partial_{x_{j}}\left(T_{a}-\right.$ $\left.\left(T_{a}\right)^{*}\right)$ map $H^{s+\frac{1}{2} \log }$ into $H^{s-\frac{1}{2} \log }$ and satisfy

$$
\begin{aligned}
& \left\|\left(T_{a}-\left(T_{a}\right)^{*}\right) \partial_{x_{j}} u\right\|_{H^{s-\frac{1}{2} \log }} \leq C\|a\|_{L L}\|u\|_{H^{s+\frac{1}{2} \log }}, \\
& \left\|\partial_{x_{j}}\left(T_{a}-\left(T_{a}\right)^{*}\right) u\right\|_{H^{s-\frac{1}{2} \log }} \leq C\|a\|_{L L}\|u\|_{H^{s+\frac{1}{2} \log }} .
\end{aligned}
$$

Proof. - The $S_{k} a$ are real valued, since $a$ is real, and the $\Delta_{k}$ are self adjoint, thus

$$
\left(T_{a}\right)^{*} u=\sum_{k=3}^{\infty} \Delta_{k}\left(\left(S_{k-3} a\right) u\right) .
$$

Therefore, one has

$$
\left(T_{a}-\left(T_{a}\right)^{*}\right)=\sum\left[S_{k-3} a, \Delta_{k}\right]=\sum\left[S_{k-3} a, \Delta_{k}\right] \Psi_{k}
$$

where $\Psi_{k}$ is a Fourier multiplier with symbol $\psi_{k}=\psi\left(2^{-k} \xi\right)$ and $\psi$ is supported in a suitable annulus. Using again [4] (see (3.17)) yields

$$
\left\|\left[S_{k-3} a, \Delta_{k}\right] \partial_{x_{j}} \Psi_{k} u\right\|_{L^{2}} \leq C(k+1)\|a\|_{L L}\left\|\Psi_{k} u\right\|_{L^{2}},
$$

and a similar estimate when the derivative is on the left of the commutator. Since the spectrum of $\left[S_{k-3} a, \Delta_{k}\right] \Psi_{k} u$ is contained in an annulus of size $\approx 2^{k}$, this implies (3.20).

Proposition 3.9. - If a and b belong to $L^{\infty} \cap L L$, then $\left(T_{a} T_{b}-T_{a b}\right) \partial_{x_{j}}$ maps $H^{s+\frac{1}{2} \log }$ into $H^{s-\frac{1}{2} \log }$ and

$$
\left\|\left(T_{a} T_{b}-T_{a b}\right) \partial_{x_{j}} u\right\|_{H^{s-\frac{1}{2} \log }} \leq C\left(\|a\|_{L L}\|b\|_{L^{\infty}}+\|b\|_{L L}\|a\|_{L^{\infty}}\right)\|u\|_{H^{s+\frac{1}{2} \log }} .
$$

Proof. - By Proposition 3.4, it is sufficient to prove the estimate for any paraproduct $T^{N}$. One has

$$
T_{a}^{N} T_{b}^{N} \partial_{x_{j}} u=\sum_{k \geq N} \sum_{l \geq N} S_{k-N} a \Delta_{k}\left(S_{l-N} b \Delta_{l} \partial_{x_{j}} u\right) .
$$

In this sum, terms with $|l-k| \leq 2$ vanish, because of the spectral localization of $S_{l-N} b \Delta_{l} \partial_{x_{j}}$. The commutators $\left[\Delta_{k}, S_{l-N} b\right]$ contribute to terms which are estimated as in (3.18):

$$
\left\|\left[\Delta_{k}, S_{l-N} b\right] \Delta_{l} \partial_{x_{j}} u\right\|_{L^{2}} \leq C(k+1)\|b\|_{L L}\left\|\Delta_{l} u\right\|_{L^{2}} .
$$

If $N$ is large enough, the spectrum of the corresponding term is contained in an annulus of size $\approx 2^{k}$ and hence the commutators contribute to an error term in (3.21). Therefore, it is sufficient to estimate

$$
\sum_{k \geq N} \sum_{l \geq N}\left(S_{k-N} a S_{l-N} b-S_{k-N}(a b)\right) \Delta_{k} \Delta_{l} \partial_{x_{j}} u .
$$

Again, only terms with $|l-k| \leq 2$ contribute to the sum. Using (3.5), one has

$$
\begin{aligned}
\left\|a-S_{k-N} a\right\|_{L^{\infty}} & \leq C(k+1) 2^{-k}\|a\|_{L L}, \\
\left\|b-S_{l-N} b\right\|_{L^{\infty}} & \leq C(k+1) 2^{-k}\|b\|_{L L}, \\
\left\|a b-S_{k-N}(a b)\right\|_{L^{\infty}} & \leq C(k+1) 2^{-k}\|a b\|_{L L} .
\end{aligned}
$$

Thus

$$
\left\|S_{k-N} a S_{l-N} b-S_{k-N}(a b)\right\|_{L^{\infty}} \leq C(k+1) 2^{-k}\left(\|a\|_{L L}\|b\|_{L^{\infty}}+\|a\|_{L^{\infty}}\|b\|_{L L}\right) .
$$


Since the terms in the sum (3.22) have their spectrum in annuli of size $\approx 2^{k}$, this implies that this sum belongs to $H^{0-\frac{1}{2} \log }$ when $u \in H^{0+\frac{1}{2} \log }$, with an estimate similar to (3.21).

\subsection{Positivity estimates}

The paradifferential calculus sketched above is well adapted to the analysis of high frequencies but does not take into account the low frequencies. For instance, the positivity of the function $a$ does not imply the positivity of the operator $T_{a}$ in $L^{2}$, only the positivity up to a smoothing operator. However, in the derivation of energy estimates, such positivity results are absolutely necessary. To avoid a separate treatment of low frequencies, we introduce modified paraproducts for which positivity results hold (we could also introduce weighted paraproducts as in $[10,11,12])$.

Consider a nonnegative integer $\nu$ and define the modified paraproducts

$$
P_{a}^{\nu} u=\sum_{k=0}^{\infty} S_{\max \{\nu, k-3\}} a \Delta_{k} u=S_{\nu} a S_{\nu+2} u+\sum_{k=\nu}^{\infty} S_{k} a \Delta_{k+3} u .
$$

Then

$$
P_{a}^{\nu} u-T_{a} u=\sum_{k=0}^{\nu+2} \sum_{j=\max \{0, k-2\}}^{\nu} \Delta_{j} a \Delta_{k} u
$$

and

$$
a u-P_{a}^{\nu} u=\sum_{j=\nu+1}^{\infty} \Delta_{j} a S_{j+2} u .
$$

The difference (3.24) concerns only low frequencies, and therefore the results of Propositions 3.7,3.8 and 3.9 are valid if one substitutes $P_{a}^{\nu}$ in place of $T_{a}$, at the cost of additional error terms. In particular, (3.24) and (3.25) immediately imply the following estimates:

Lemma 3.10. - i) There is a constant $C$ such that for all $\nu, a \in L^{\infty}$ and all $u \in L^{2}$,

$$
\left\|\left(P_{a}^{\nu}-T_{a}\right) \partial_{x_{j}} u\right\|_{L^{2}}+\left\|\partial_{x_{j}}\left(P_{a}^{\nu}-T_{a}\right) u\right\|_{L^{2}} \leq C 2^{\nu}\|a\|_{L^{\infty}}\|u\|_{L^{2}} .
$$

ii) There is a constant $C_{0}$ such that for all $\nu$, for all $a \in L L$ and all $u \in L^{2}$,

$$
\left\|a u-P_{a}^{\nu} u\right\|_{L^{2}} \leq C_{0} \nu 2^{-\nu}\|a\|_{L L}\|u\|_{L^{2}} .
$$

We will also use the following extension of Proposition 3.8:

Proposition 3.11. - If $a \in L^{\infty} \cap L L$ is real valued, then $\left(P_{a}^{\nu}-\left(P_{a}^{\nu}\right)^{*}\right) \partial_{x_{j}}$ and $\partial_{x_{j}}\left(P_{a}^{\nu}-\right.$ $\left.\left(P_{a}^{\nu}\right)^{*}\right)$ map $H^{0+\frac{1}{2} \log }$ into $H^{0-\frac{1}{2} \log }$ and

$$
\begin{aligned}
& \left\|\left(P_{a}^{\nu}-\left(P_{a}^{\nu}\right)^{*}\right) \partial_{x_{j}} u\right\|_{H^{0-\frac{1}{2} \log }} \leq C\|a\|_{L L}\left(\|u\|_{H^{0+\frac{1}{2} \log }}+\nu\|u\|_{L^{2}}\right), \\
& \left\|\partial_{x_{j}}\left(P_{a}^{\nu}-\left(P_{a}^{\nu}\right)^{*}\right) u\right\|_{H^{0-\frac{1}{2} \log }} \leq C\|a\|_{L L}\left(\|u\|_{H^{0+\frac{1}{2} \log }}+\nu\|u\|_{L^{2}}\right) .
\end{aligned}
$$


Proof. - One has

$$
\left(P_{a}^{\nu}-\left(P_{a}^{\nu}\right)^{*}\right) \partial_{x_{j}} u=\left[S_{\nu} a, S_{\nu+2}\right] \partial_{x_{j}} u+\sum_{k \geq \nu}\left[S_{k} a, \Delta_{k+3}\right] \partial_{x_{j}} u .
$$

The sum over $k$ is treated exactly as in the proof of Proposition 3.8 and contibutes to the same error term. Using again Theorem 35 of [4], the $L^{2}$ norm of the first term is estimated by

$$
C\left\|\nabla_{x} S_{\nu} a\right\|_{L^{\infty}}\|u\|_{L^{2}} \leq C(\nu+1)\|a\|_{L L}\|u\|_{L^{2}}
$$

and contibutes to the second error term in (3.28). When the derivative is on the left, the proof is similar.

Moreover, a comparison of $P_{a}^{\nu} u$ with au immediately implies the following positivity estimate.

COROLLARY 3.12. - There is a constant $c_{0}$, such that for any positive LL-function a such that $\delta=\min a(x)>0$, all $\nu$ such that $\nu 2^{-\nu} \leq c_{0} \delta /\|a\|_{L L}$, and $u \in L^{2}\left(\mathbb{R}^{n}\right)$,

$$
\operatorname{Re}\left(P_{a}^{\nu} u, u\right)_{L^{2}} \geq \frac{\delta}{2}\|u\|_{L^{2}}^{2}
$$

Here, $(\cdot, \cdot)_{L^{2}}$ denotes the scalar product in $L^{2}$. This estimate extends to vector valued functions $u$ and matrices $a$, provided that $a$ is symmetric and positive.

\subsection{The time dependent case}

In the sequel we will consider functions of $(t, x) \in[0, T] \times \mathbb{R}^{n}$, considered as functions of $t$ with values in various spaces of functions of $x$. In particular we denote by $T_{a}$ the operator acting for each fixed $t$ as $T_{a(t)}$ :

$$
\left(T_{a} u\right)(t)=\sum_{k=3}^{\infty} S_{k-3}\left(D_{x}\right) a(t) \Delta_{k}\left(D_{x}\right) u(t) .
$$

The Propositions 3.4, 3.7, 3.8 and 3.9 apply for each fixed $t$. There are similar definitions for the modified paraproducts $P_{a}^{\nu}$; further, Lemma 3.10 and Corollary 3.12 apply for fixed $t$.

When $a$ is a Lipschitz function of $t$, the definition (3.30) immediately implies that

$$
\left[\partial_{t}, T_{a}\right]=T_{\partial_{t} a}, \quad\left[\partial_{t}, P_{a}^{\nu}\right]=P_{\partial_{t} a}^{\nu}
$$

When $a$ is only Log Lipschitz this formula does not make sense, since $\partial_{t} a$ is not defined as a function. The idea, already used in $[5,6]$, is that it is sufficient to commute $\partial_{t}$ with time regularization of $a$. In our context, this simply means that in (3.30), we will replace the term $S_{k-3} a$, which is a spatial regularization of $a$, by a space-time regularization, namely $S_{k-3} a_{k}$ where $a_{k}$ is a suitable time mollification of $a$. We now give the details for $P^{\nu}$, as we will need them in the next section.

Introduce the mollifiers

$$
\jmath_{k}(t)=2^{k} \jmath\left(2^{k} t\right)
$$

where $\jmath \in C_{0}^{\infty}(\mathbb{R})$ is non negative, with integral over $\mathbb{R}$ equal to 1 .

$4^{\mathrm{e}}$ SÉRIE - TOME $41-2008-\mathrm{N}^{\mathrm{o}} 2$ 
Definition 3.13. - Given $a \in L^{\infty} \cap L L\left(\left[0, T_{0}\right] \times \mathbb{R}^{n}\right)$, define

$$
a_{k}(t, x)=\jmath_{k} *_{t} \tilde{a}=\int \jmath_{k}(t-s) \tilde{a}(s, x) d s
$$

where $\tilde{a}$ is the LL extension of a given by

$$
\tilde{a}(t, x)=a(0, x), \quad t \leq 0, \quad \tilde{a}(t, x)=a\left(T_{0}, x\right), \quad t \geq T_{0} .
$$

Next, for fixed $t$, the operator $\widetilde{P}_{a(t)}^{\nu}$ is defined by

$$
\widetilde{P}_{a(t)}^{\nu} u=S_{\nu} a_{\nu} S_{\nu+2} u+\sum_{k=\nu}^{\infty} S_{k} a_{k} \Delta_{k+3} u
$$

We denote by $\widetilde{P}_{a}^{\nu}$ the operator acting on functions of $(t, x)$ by $\left(\widetilde{P}_{a}^{\nu} u\right)(t)=\widetilde{P}_{a(t)}^{\nu} u(t)$.

Proposition 3.14. - Let $a \in L^{\infty} \cap L L\left(\left[0, T_{0}\right] \times \mathbb{R}^{n}\right)$. Then for each $t \in\left[0, T_{0}\right]$, the operators $R_{1}(t)=\left(P_{a(t)}^{\nu}-\widetilde{P}_{a(t)}^{\nu}\right) \partial_{x_{j}}, R_{2}(t)=\partial_{x_{j}}\left(P_{a(t)}^{\nu}-\widetilde{P}_{a(t)}^{\nu}\right), R_{3}(t)=\left(\left(\widetilde{P}_{a(t)}^{\nu}\right)^{*}-\right.$ $\left.\widetilde{P}_{a(t)}^{\nu}\right) \partial_{x_{j}}, R_{4}(t)=\partial_{x_{j}}\left(\left(\widetilde{P}_{a(t)}^{\nu}\right)^{*}-\widetilde{P}_{a(t)}^{\nu}\right)$, and $R_{5}(t)=\left[D_{t}, \widetilde{P}_{a}^{\nu}\right](t)$ map $H^{0+\frac{1}{2} \log }$ into $H^{0-\frac{1}{2} \log }$ and there is a constant $C$ such that for all $t \in\left[0, T_{0}\right]$ and for $k=1, \ldots, 5$,

$$
\left\|R_{k} u\right\|_{H^{0-\frac{1}{2} \log }} \leq C\|a\|_{L L}\left(\|u\|_{H^{0+\frac{1}{2} \log }}+\nu\|u\|_{L^{2}}\right) .
$$

Proof. - a) First, we recall several estimates from [6]. For $a \in L L\left(\left[0, T_{0}\right] \times \mathbb{R}^{n}\right)$ the difference $a-a_{k}$ satisfies

$$
\begin{aligned}
\left|a(t, x)-a_{k}(t, x)\right| & \leq C(k+1) 2^{-k}\|a\|_{L L}, \\
\left|\partial_{t} a_{k}(t, x)\right| & \leq C(k+1)\|a\|_{L L},
\end{aligned}
$$

with $C$ independent of $t$ and $x$. In particular, we note that

$$
\left\|S_{k}\left(a(t)-a_{k}(t)\right)\right\|_{L^{\infty}} \leq C(k+1) 2^{-k}\|a\|_{L L} .
$$

b) In accordance with (3.35), for $l=1,2,5$, we split $R_{l}$ into two terms

$$
R_{l}(t) u=B_{l} u+H_{l} u, \quad H_{l} u=\sum_{k \geq \nu} w_{k}
$$

with $B_{l} u$ spectrally supported in the ball of radius $2^{\nu+4}$ and with $w_{k}$ spectrally supported in an annulus $|\xi| \approx 2^{k}$. For $R_{1}$,

$$
B_{1} u=S_{\nu}\left(a(t)-a_{\nu}(t)\right) S_{\nu+2} \partial_{x_{j}} u, \quad w_{k}=S_{k}\left(a(t)-a_{k}(t)\right) \Delta_{k+3} \partial_{x_{j}} u .
$$

With (3.39), this implies that

$$
\left\|B_{1} u\right\|_{L^{2}} \leq C(\nu+1)\|a\|_{L L}\|u\|_{L^{2}}
$$

and

$$
\left\|w_{k}\right\|_{L^{2}} \leq C(k+1)\|a\|_{L L}\left\|\Delta_{k+3} u\right\|_{L^{2}}
$$

implying that

$$
\left\|H_{1} u\right\|_{H^{0-\frac{1}{2} \log }} \leq C\|a\|_{L L}\|u\|_{H^{0+\frac{1}{2} \log }} .
$$


For $R_{2}$, the analysis is similar. One has

$$
B_{2} u=\partial_{x_{j}}\left(S_{\nu}\left(a(t)-a_{\nu}(t)\right) S_{\nu+2} u\right), \quad w_{k}=\partial_{x_{j}}\left(S_{k}\left(a(t)-a_{k}(t)\right) \Delta_{k+3} u\right) .
$$

Thanks to the spectral localization, the estimates for $B_{2} u$ and $w_{k}$ are the same as in the case of $R_{1}$, implying that

$$
\begin{aligned}
\left\|B_{2} u\right\|_{L^{2}} & \leq C(\nu+1)\|a\|_{L L}\|u\|_{L^{2}} \\
\left\|H_{2} u\right\|_{H^{0-\frac{1}{2} \log }} & \leq C\|a\|_{L L}\|u\|_{H^{0+\frac{1}{2} \log }} .
\end{aligned}
$$

c) For $k=5$ we write (3.40) with

$$
B_{5} u=S_{\nu}\left(\partial_{t} a_{\nu}(t)\right) \Delta_{\nu+2} u, \quad w_{k}=S_{k}\left(\partial_{t} a_{k}(t)\right) \Delta_{k+3} u .
$$

Thus the estimates (3.38) imply

$$
\begin{aligned}
\left\|B_{5} u\right\|_{L^{2}} & \leq C(\nu+1)\|a\|_{L L}\|u\|_{L^{2}} \\
\left\|H_{5} u\right\|_{H^{0-\frac{1}{2} \log }} & \leq C\|a\|_{L L}\|u\|_{H^{0+\frac{1}{2} \log }} .
\end{aligned}
$$

d) One has

$$
R_{3}(t)=R_{1}(t)+R_{2}^{*}(t)+\left(\left(P_{a(t)}^{\nu}\right)^{*}-P_{a(t)}^{\nu}\right) \partial_{x_{j}} .
$$

The third term is estimated in Proposition 3.11. The operators $R_{1}$ and $R_{2}^{*}=B_{2}^{*}+H_{2}^{*}$ are estimated in part b), implying that $R_{3}$ satisfies (3.36) for $k=3$. The proof for $R_{4}=R_{3}^{*}=$ $R_{1}^{*}+R_{2}+\partial_{x_{j}}\left(\left(P_{a(t)}^{\nu}\right)^{*}-P_{a(t)}^{\nu}\right)$ is similar.

This finishes the proof of the proposition.

Lemma 3.15. - There is a constant $C_{0}$ such that for any $a \in L L\left(\left[0, T_{0}\right] \times \mathbb{R}^{n}\right), u \in L^{2}\left(\mathbb{R}^{n}\right)$, $\nu \geq 0$ and all $t \in\left[0, T_{0}\right]$, one has

$$
\left\|a(t) u-\widetilde{P}_{a(t)}^{\nu} u\right\|_{L^{2}} \leq C_{0} \nu 2^{-\nu}\|a\|_{L L}\|u\|_{L^{2}} .
$$

Proof. - We have

$$
a u-\widetilde{P}_{a}^{\nu} u=\left(a-S_{\nu} a_{\nu}\right) S_{\nu+2} u+\sum_{k=\nu}^{\infty}\left(a-S_{k} a_{k}\right) \Delta_{k+3} u .
$$

Combining (3.5) and (3.39), we see that

$$
\left\|a(t)-S_{k} a_{k}(t)\right\|_{L^{\infty}} \leq C k 2^{-k}\|a\|_{L L} .
$$

This implies (3.43).

The lemma immediately implies the following positivity estimate.

COROLlaRY 3.16. - There is a constant $c_{0}$, such that for any positive LL-function a such that $\delta=\min a(t, x)>0$, all $\nu$ such that $\nu 2^{-\nu} \leq c_{0} \delta /\|a\|_{L L}$, and $u \in L^{2}\left(\mathbb{R}^{n}\right)$,

$$
\operatorname{Re}\left(P_{a(t)}^{\nu} u, u\right)_{L^{2}\left(\mathbb{R}^{n}\right)} \geq \frac{\delta}{2}\|u\|_{L^{2}\left(\mathbb{R}^{n}\right)}^{2} .
$$

The same result holds for vector valued functions $u$ and definite positive square matrices $a$.

Finally, we quote the following commutation result which will be needed in the next section.

$4^{\mathrm{e}}$ SÉRIE - TOME $41-2008-\mathrm{N}^{\mathrm{o}} 2$ 
Proposition 3.17. - Suppose that $a \in L L\left(\left[0, T_{0}\right] \times \mathbb{R}^{n}\right)$. Then $\Lambda^{\frac{1}{2}}\left[\widetilde{P}_{a(t)}^{\nu}, \Lambda^{\frac{1}{2}}\right]$ and $\left[\widetilde{P}_{a(t)}^{\nu}, \Lambda^{\frac{1}{2}}\right] \Lambda^{\frac{1}{2}}$ are bounded in $L^{2}$ and satisfy

$$
\left\|\Lambda^{\frac{1}{2}}\left[\widetilde{P}_{a(t)}^{\nu}, \Lambda^{\frac{1}{2}}\right] u\right\|_{L^{2}}+\left\|\left[\widetilde{P}_{a(t)}^{\nu}, \Lambda^{\frac{1}{2}}\right] \Lambda^{\frac{1}{2}} u\right\|_{L^{2}} \leq C\left(\nu^{2} 2^{-\nu}\|a\|_{L L}+\nu\|a\|_{L^{\infty}}\right)\|u\|_{L^{2}} .
$$

Proof. - Thanks to the spectral localization, the low frequency part $S_{\nu} a_{\nu} S_{\nu+2}$ in $\widetilde{P}_{a}^{\nu}$ contributes to terms whose $L^{2}$ norm is bounded by

$$
C \nu\|u\|_{L^{2}} .
$$

The commutator with the high frequency part reads

$$
\sum_{k \geq \nu}\left[\Lambda^{\frac{1}{2}}, S_{k} a_{k}\right] \Delta_{k+3} u
$$

We argue as in the proof of Proposition 3.7 and write

$$
\left[\Lambda^{\frac{1}{2}}, S_{k} a_{k}\right] \Delta_{k+3}=(k+1)^{\frac{1}{2}}\left[H_{k}, S_{k} a_{k}\right] \Delta_{k+3}
$$

where the symbol of $H_{k}$ is $h_{k}(\xi)=(k+1)^{-\frac{1}{2}}(\log (2+|\xi|))^{\frac{1}{2}} \varphi\left(2^{-k} \xi\right)$ and $\varphi$ is supported in a suitable fixed annulus. Note that the family $\left\{h_{k}\right\}$ is bounded in the space of symbols of degree 0. By (3.17), one has

$$
\left\|\left[H_{k}, S_{k} a(t)\right] \Lambda^{\frac{1}{2}} \Delta_{k+3} u\right\|_{L^{2}} \leq C(k+1) 2^{-k}\left\|\nabla_{x} S_{k} a_{k}(t)\right\|_{L^{\infty}}\left\|\Delta_{k} u\right\|_{L^{2}} .
$$

Since $\nabla_{x} S_{k} a_{k}=\left(\nabla_{x} S_{k} a\right) * \jmath_{k}$, its $L^{\infty}$ norm is bounded by $C k\|a\|_{L L}$. Adding up, and using the spectral localization, these terms contribute by a function whose $L^{2}$ norm is bounded by $C \nu^{2} 2^{-\nu}\|a\|_{L L}\|u\|_{L^{2}}$.

When $\Lambda^{\frac{1}{2}}$ is on the left of the commutator, the analysis is similar.

\section{Proof of the main results}

\subsection{The main estimate}

We consider the operator (2.1) with coefficients which satisfy (2.4), (2.5) and (2.6). We fix $\theta<\theta_{1}$ in $] 1-\alpha, \alpha[$, and with $\lambda$ to be chosen later, we introduce the notation

$$
s(t)=\theta+t \lambda \text {. }
$$

Recall that

$$
T=\min \left\{T_{0}, \frac{\theta_{1}-\theta}{\lambda}\right\} .
$$

Note that for $t \in[0, T], s(t)$ remains in $\left.\left[\theta, \theta_{1}\right] \subset\right] 1-\alpha, \alpha[$.

We will consider solutions of the Cauchy problem

$$
L u=f, \quad u_{\mid t=0}=u_{0}, \quad X u_{\mid t=0}=u_{1}
$$

with

$$
\begin{gathered}
u \in \mathcal{H}_{1-\theta+\frac{1}{2} \log , \lambda}(T), \quad \partial_{t} u \in \mathcal{H}_{-\theta+\frac{1}{2} \log , \lambda}(T), \\
u_{0} \in H^{1-\theta}\left(\mathbb{R}^{n}\right), \quad u_{1} \in H^{-\theta}\left(\mathbb{R}^{n}\right), \\
f=f_{1}+f_{2}, \quad f_{1} \in \mathcal{L}_{-\theta, \lambda}(T), \quad f_{2} \in \mathcal{H}_{-\theta-\frac{1}{2} \log , \lambda}(T),
\end{gathered}
$$


Note that if $u$ and $f$ satisfy (4.4) and (4.6), then

$$
\begin{gathered}
u \in L^{2}\left([0, T] ; H^{1-\theta_{1}}\right), \quad \partial_{t} u \in L^{2}\left([0, T] ; H^{-\theta_{1}}\right), \\
f \in L^{1}\left([0, T] ; H^{-\theta_{2}}\right)
\end{gathered}
$$

for all $\left.\theta_{2} \in\right] \theta_{1}, \alpha[$, so that the meaning of the Cauchy condition is clear.

The main step in the proof of Theorem 2.6 is the following:

THeOREm 4.1. - There is a $\lambda_{0} \geq 0$ of the form (2.30) such that for $\lambda \geq \lambda_{0}$ there is a constant $K$ such that: for all $f, u_{0}$ and $u_{1}$ satisfying (4.5) (4.6), and all u satisfying (4.4) solution of the Cauchy problem (4.3), one has

$$
u \in \mathcal{C}_{1-\theta, \lambda}(T), \quad \partial_{t} u \in \mathcal{C}_{-\theta, \lambda}(T) .
$$

Moreover u satisfies the energy estimate (2.29).

This theorem contains two pieces of information : first an energy estimate for smooth $u$, see Propositions 4.3 and 4.4. By a classical argument, smoothing the coefficients and passing to the limit, this estimate allows for the construction of weak solutions, see Section 5.2. The second piece of information contained in the theorem is a "weak=strong" type result showing that for data as in the theorem, any (weak) solution $u$ satisfying (4.4) is the limit of smooth (approximate) solutions, in the norm given by the left hand side of the energy estimate, implying that $u$ satisfies the additional smoothness (4.9) and the energy estimate. This implies uniqueness of weak solutions.

The idea is to get an energy estimate by integration by parts, from the analysis of

$$
2 \operatorname{Re}\left\langle L u, e^{-2 \gamma t}\left(1-\Delta_{x}\right)^{-s(t)} X u\right\rangle
$$

where $\langle\cdot, \cdot\rangle$ denotes the $L^{2}$ scalar product in $\mathbb{R}^{n}$ extended to the Hermitian symmetric duality $H^{\sigma} \times H^{-\sigma}$ for all $\sigma \in \mathbb{R}$, and $\Delta_{x}$ denote the Laplace operator on $\mathbb{R}^{d}$. This extends the analysis of [6] where $X=\partial_{t}$. The parameter $\gamma$ is chosen at the end to absorb classical error terms (present for Lipschitz coefficients) while the parameter $\lambda$ which enters in the definition of $s(t)$, is chosen to absorb extra error terms coming from the loss of smoothness of the coefficients.

To prove Theorem 4.1, the first idea would be to mollify the equation. However, the lack of smoothness of the coefficients does not allow us to use this method directly and we cannot prove that the weak solutions are limits of exact smooth solutions. Instead, the idea is to write the equation as a system (2.16) for $(u, v)$ and mollify this system. This leads to the consideration of the equations:

$$
\left\{\begin{array}{l}
Z v+\tilde{b}_{0} v=\tilde{L}_{2} u-\tilde{L}_{1} u-\tilde{d} u+f \\
Y u+\tilde{c}_{0} u=v / a_{0}+g
\end{array}\right.
$$

In this form, the commutator of spatial mollifiers with $\partial_{t}$ are trivial, and we can prove that weak solutions of (4.11) are limits of smooth solutions, $\left(u^{\varepsilon}, v^{\varepsilon}\right)$ with $g^{\varepsilon} \neq 0$, which thus do not correspond to exact solutions $u^{\varepsilon}$ of (4.3). 
Notations. It is important for our purpose to keep track of the dependence of the various constants on the Log-Lipschitz norms. In particular we will use the notations $\delta_{0}, \delta_{1}$ of (2.8) and $A_{L L}, A_{L^{\infty}}, B$ of (2.9) (2.10). To simplify the exposition, we will denote by $C, K_{0}$ and $K$ constants which may vary from one line to another, $C$ denoting universal constants depending only on the paradifferential calculus; $K_{0}$ depending also on $A_{L^{\infty}} / \delta_{0} ; K$, still independent of the parameters $(\gamma, \varepsilon)$, but dependent also on $\delta_{0}, \delta_{1}, \theta_{0}, \theta_{1}$ and the various norms of the coefficients.

\subsection{Estimating $v$}

First, we give estimates that link $v$ and $\partial_{t} u$.

Lemma 4.2. - Suppose that u satisfies (4.4). Then $v=X u+c_{0} u$ belongs to the space $\mathcal{H}_{-\theta+\frac{1}{2} \log , \lambda}(T) \subset L^{2}\left([0, T] ; H^{-\theta_{1}}\right)$ and for almost all $t$,

$$
\begin{aligned}
\|v(t)\|_{H^{-s(t)+\frac{1}{2} \log } \leq} & C A_{L^{\infty}}\left(\|u(t)\|_{H^{1-s(t)+\frac{1}{2} \log }}+\left\|\partial_{t} u(t)\right\|_{H^{-s(t)+\frac{1}{2} \log }}\right) \\
& +C\left(A_{L L}+B\right)\left(\|u(t)\|_{H^{1-s(t)}}+\left\|\partial_{t} u(t)\right\|_{H^{-s(t)}}\right), \\
\left\|\partial_{t} u(t)\right\|_{H^{-s(t)+\frac{1}{2} \log } \leq} & K_{0}\|u(t)\|_{H^{1-s(t)+\frac{1}{2} \log }}+\frac{C}{\delta_{0}}\|v(t)\|_{H^{-s(t)+\frac{1}{2} \log }} \\
& +K\left(\|u(t)\|_{H^{1-s(t)}}+\|v(t)\|_{H^{-s(t)}}\right) .
\end{aligned}
$$

There are similar estimates in the spaces $H^{s}$ without the $\frac{1}{2} \log$.

If in addition $L u=f$ with $f$ satisfying (4.6), then $\partial_{t} v \in L^{1}\left([0, T] ; H^{-1-\theta_{1}}\right)$.

Proof. - a) First, we note that the multiplication $(a, u) \mapsto a u$ is continuous from $\left(L^{\infty} \cap\right.$ $L L)\left([0, T] \times \mathbb{R}^{n}\right) \times \mathcal{H}_{-\theta+\frac{1}{2} \log , \lambda}(T)$ to $\mathcal{H}_{-\theta+\frac{1}{2} \log , \lambda}(T)$. Indeed, the corresponding norm estimate of the product is clear for smooth $u$, from (3.15) integrated in time. The claim follows by density. In particular, this shows that $a_{0} \partial_{t} u$ and the $a_{j} \partial_{x_{j}} u$ belong to $\mathcal{H}_{-\theta+\frac{1}{2} \log , \lambda}(T)$. Similarly, the estimate

$$
\|b u(t)\|_{H^{-s(t)+\frac{1}{2} \log }} \leq C\|b u(t)\|_{H^{1-s(t)}} \leq C\|b\|_{C^{\alpha}}\|u(t)\|_{H^{1-s(t)}}
$$

implies that $c_{0} u \in \mathcal{H}_{-\theta+\frac{1}{2} \log , \lambda}(T)$. Therefore $v \in \mathcal{H}_{-\theta+\frac{1}{2} \log , \lambda}(T)$ and the estimate (4.12) holds. The proof of (4.13) is similar, noting that

$$
\partial_{t} u=\frac{1}{a_{0}} v-\sum_{j=1}^{d} \frac{a_{j}}{a_{0}} \partial_{x_{j}} u-\frac{c_{0}}{a_{0}} u .
$$

b) As in the proof of Lemma 2.2, we see that the equation implies that

$$
\partial_{t} v=f-\sum_{j=1}^{d} \partial_{x_{j}}\left(\tilde{a}_{j} v\right)-\tilde{b}_{0} v+\tilde{L}_{2} u-\tilde{L}_{1} u-\tilde{d} u .
$$

The conservative form of $\tilde{L}_{2}$ and the multiplicative properties above show that

$$
\partial_{x_{j}}\left(\tilde{a}_{j} v\right), \tilde{L}_{2} u \in \mathcal{H}_{-\theta-1+\frac{1}{2} \log , \lambda}(T) \subset L^{2}\left([0, T] ; H^{-1-\theta_{1}}\right) .
$$

Similarly, $\tilde{L}_{1} u$ and $\tilde{b}_{0} v$ belong to $\mathcal{H}_{-\theta+\frac{1}{2} \log , \lambda}(T)$ and thus to $L^{2}\left([0, T] ; H^{-\theta_{1}}\right)$. The last term $\tilde{u}$ is in $L^{2}$. Therefore, $\partial_{t} v-f \in L^{2}\left([0, T] ; H^{-1-\theta_{1}}\right)$. Since $f \in L^{1}\left([0, T] ; H^{-\theta_{2}}\right)$ for $\left.\theta_{2} \in\right] \theta_{1}, \alpha[$, the lemma follows. 
Next, we give a-priori estimates in the space $\mathcal{H}_{-\theta+\frac{1}{2} \log , \lambda}(T) \cap \mathcal{C}_{-\theta, \lambda}(T)$ for smooth solutions of

$$
\left(Z+\tilde{c}_{0}\right) v=\varphi, \quad v_{\mid t=0}=v_{0}
$$

We define the operators

$$
(Q v)(t)=\left(1-\Delta_{x}\right)^{-s(t) / 2} v(t), \quad\left(Q_{\gamma} v\right)(t)=e^{-\gamma t}(Q v)(t) .
$$

Proposition 4.3. - Suppose that $v \in L^{2}\left([0, T] ; H^{1}\right)$ and $\partial_{t} v \in L^{1}\left([0, T] ; L^{2}\right)$. Then the functions $v_{\gamma}(t):=Q_{\gamma}$ v belong to $C^{0}\left([0, T], L^{2}\right)$ and satisfy

$$
\begin{aligned}
\left\|v_{\gamma}(t)\right\|_{L^{2}}^{2} & +2 \int_{0}^{t}\left\|(\gamma+\lambda \Lambda)^{1 / 2} v_{\gamma}\left(t^{\prime}\right)\right\|_{L^{2}}^{2} d t^{\prime} \\
& \leq 2 \int_{0}^{t}\left\langle\left(Z+\tilde{c}_{0}\right) v\left(t^{\prime}\right), Q_{\gamma}^{2}\left(t^{\prime}\right) v\left(t^{\prime}\right)\right\rangle d t^{\prime}+\left\|v_{\gamma}(0)\right\|_{L^{2}}^{2}+\int_{0}^{t} F\left(t^{\prime}\right) d t^{\prime}
\end{aligned}
$$

with

$$
F\left(t^{\prime}\right) \leq K_{0} \frac{A_{L L}}{\delta_{0}}\left\|e^{-\gamma t^{\prime}} \Lambda^{1 / 2} v\left(t^{\prime}\right)\right\|_{H^{-s\left(t^{\prime}\right)}}^{2}+K\left\|v\left(t^{\prime}\right)\right\|_{H^{-s\left(t^{\prime}\right)}}^{2} .
$$

Proof. - a) Since $v \in L^{2}\left([0, T] ; H^{1}\right)$ and $\partial_{t} v \in L^{1}\left([0, T] ; L^{2}\right)$, we have

$$
\partial_{t} Q_{\gamma} v=Q_{\gamma} \partial_{t} v-(\gamma+\lambda \Lambda) Q_{\gamma} v \in L^{1}\left([0, T] ; L^{2}\right)
$$

as immediately seen using the spatial Fourier transform. Moreover, $v_{\gamma}=Q_{\gamma} v \in C^{0}\left([0, T] ; L^{2}\right)$ and satisfies the following identity

$$
\left\|v_{\gamma}(t)\right\|_{L^{2}}^{2}-\left\|v_{\gamma}(0)\right\|_{L^{2}}^{2}=2 \operatorname{Re} \int_{0}^{t}\left\langle\partial_{t} Q_{\gamma} v, Q_{\gamma} v\right\rangle d t^{\prime}
$$

Thus,

$$
\begin{aligned}
& 2 \operatorname{Re} \int_{0}^{t}\left\langle\partial_{t} v, Q_{\gamma}^{2} v\right\rangle d t^{\prime}=2 \operatorname{Re} \int_{0}^{t}\left\langle Q_{\gamma} \partial_{t} v, Q_{\gamma} v\right\rangle d t^{\prime} \\
& \quad=\left\|v_{\gamma}(t)\right\|_{L^{2}}^{2}-\left\|v_{\gamma}(0)\right\|_{L^{2}}^{2}+2 \int_{0}^{t}\left\|(\gamma+\lambda \Lambda)^{1 / 2} v_{\gamma}\left(t^{\prime}\right)\right\|_{L^{2}}^{2} d t^{\prime}
\end{aligned}
$$

b) Next we consider the terms $\partial_{x_{j}}\left(\tilde{a}_{j} v\right)$. We note that they belong to $L^{2}\left([0, T] ; H^{-\sigma}\right)$ for all $\sigma>0$. In particular, since $s(t) \geq \theta>0$, we note that the pairing

$$
\left\langle\partial_{x_{j}}\left(\tilde{a}_{j} v\right), Q_{\gamma}^{2} v\right\rangle
$$

is well defined. We give an estimate for

using the decomposition

$$
2 \operatorname{Re} \int_{0}^{t}\left\langle\partial_{x_{j}}\left(\tilde{a}_{j} v\right), Q_{\gamma}^{2} v\right\rangle d t^{\prime}
$$

By Proposition 3.4 it follows

$$
\tilde{a}_{j} v=T_{\tilde{a}_{j}} v+R_{\tilde{a}_{j}} v
$$

$$
\left\|R_{\tilde{a}_{j}} v(t)\right\|_{H^{1-s(t)-\frac{1}{2} \log }} \leq C\left\|\tilde{a}_{j}\right\|_{L L}\|v(t)\|_{H^{-s(t)+\frac{1}{2} \log }}
$$

since $\left.s(t) \in\left[\theta, \theta_{1}\right] \subset\right] 0,1[$. Moreover,

$$
\left\|Q_{\gamma}^{2} v(t)\right\|_{H^{s(t)+\frac{1}{2} \log }} \leq C e^{-2 \gamma t}\|v(t)\|_{H^{-s(t)+\frac{1}{2} \log }} .
$$


Thus

$$
\begin{aligned}
\left|\left\langle\partial_{x_{j}} R_{\tilde{a}_{j}} v(t), Q_{\gamma, \varepsilon}^{2} v(t)\right\rangle\right| & \leq\left\|R_{\tilde{a}_{j}} v(t)\right\|_{H^{1-s(t)-\frac{1}{2} \log }}\left\|Q_{\gamma, \varepsilon}^{2} v(t)\right\|_{H^{s(t)+\frac{1}{2} \log }} \\
& \leq C\left\|\tilde{a}_{j}\right\|_{L L} e^{-2 \gamma t}\|v(t)\|_{H^{-s(t)+\frac{1}{2} \log }}^{2}
\end{aligned}
$$

It remains to consider

$$
\begin{aligned}
\operatorname{Re}\left\langle\partial_{x_{j}} T_{\tilde{a}_{j}} v, Q_{\gamma}^{2} v\right\rangle & =\operatorname{Re}\left\langle Q_{\gamma} \partial_{x_{j}} T_{\tilde{a}_{j}} v, Q_{\gamma} v\right\rangle \\
& =\operatorname{Re}\left\langle\partial_{x_{j}} T_{\tilde{a}_{j}} Q_{\gamma} v, Q_{\gamma} v\right\rangle+\operatorname{Re}\left\langle\partial_{x_{j}}\left[Q_{\gamma}, T_{\tilde{a}_{j}}\right] v, Q_{\gamma} v\right\rangle .
\end{aligned}
$$

Note that these computations make sense because $v(t) \in H^{1}$ and all the pairings are well defined. Proposition 3.7 implies that

$$
\|\left\langle\partial_{x_{j}}\left[Q_{\gamma}, T_{\tilde{a}_{j}}\right] v(t)\left\|_{0-\frac{1}{2} \log } \leq C e^{-\gamma t}\right\| \tilde{a}_{j}\left\|_{L L}\right\| v(t) \|_{-s(t)+\frac{1}{2} \log }\right.
$$

and therefore

$$
\left|\left\langle\partial_{x_{j}}\left[Q_{\gamma}, T_{\tilde{a}_{j}}\right] v(t), Q_{\gamma} v(t)\right\rangle\right| \leq C\left\|\tilde{a}_{j}\right\|_{L L} e^{-2 \gamma t}\|v(t)\|_{H^{-s(t)+\frac{1}{2} \log }}^{2} .
$$

Next, for $v_{\gamma}(t) \in H^{2-\theta_{1}}$, we have

$$
\begin{aligned}
2 \operatorname{Re}\left\langle\partial_{x_{j}} T_{\tilde{a}_{j}} v_{\gamma}, v_{\gamma}\right\rangle & =\operatorname{Re}\left\langle\left(\partial_{x_{j}} T_{\tilde{a}_{j}}-T_{\tilde{a}_{j}}^{*} \partial_{x_{j}}\right) v_{\gamma}, v_{\gamma}\right\rangle \\
& =\operatorname{Re}\left\langle\left(T_{\tilde{a}_{j}}-T_{\tilde{a}_{j}}^{*}\right) \partial_{x_{j}} v_{\gamma}, v_{\gamma}\right\rangle+\operatorname{Re}\left\langle\left[\partial_{x_{j}}, T_{\tilde{a}_{j}}\right] \partial_{x_{j}} v_{\gamma,}, v_{\gamma}\right\rangle .
\end{aligned}
$$

Using Propositions 3.8 and 3.7, one can bound both terms by the right hand side of (4.21). Adding up, we have proved that

$$
\left|2 \operatorname{Re} \int_{0}^{t}\left\langle\partial_{x_{j}}\left(\tilde{a}_{j} v\right), Q_{\gamma}^{2} v\right\rangle d t^{\prime}\right| \leq C\left\|\tilde{a}_{j}\right\|_{L L} \int_{0}^{t}\left\|e^{-\gamma t^{\prime}} \Lambda^{1 / 2} v\left(t^{\prime}\right)\right\|_{H^{-s(t)}}^{2} d t^{\prime} .
$$

c) The zero-th order term is clearly a remainder, and the multiplicative properties imply that

$$
\mid\left\langle\tilde{c}_{0} v(t), Q_{\gamma}^{2} v(t)\right\rangle \leq K\|v(t)\|_{H^{-s(t)}}^{2} .
$$

d) We note that

$$
\begin{aligned}
\left\|a_{j} / a_{0}\right\|_{L L} & \leq\left\|a_{j}\right\|_{L L}\|\| 1 / a_{0}\left\|_{L^{\infty}}+\right\| a_{j}\left\|_{L^{\infty}}\right\|\left\|1 / a_{0}\right\|_{L L} \\
& \leq \frac{A_{L L}}{\delta_{0}}+\frac{A_{L^{\infty}} A_{L L}}{\delta_{0}^{2}} \leq 2 \frac{A_{L^{\infty}} A_{L L}}{\delta_{0}^{2}}
\end{aligned}
$$

since $\delta_{0} \leq a_{0} \leq A_{L^{\infty}}$. Using identity (4.20) and the estimates of parts b) and c), implies (4.17) and so the proof of the lemma is complete.

\subsection{Estimating $\nabla_{x} u$}

We now get estimates of $\nabla_{x} u$ from the analysis of

$$
-2 \operatorname{Re}\left\langle\widetilde{L}_{2} u, Q_{\gamma}^{2} X u\right\rangle=-\sum_{j, k=1}^{n} 2 \operatorname{Re}\left\langle\partial_{x_{j}}\left(\tilde{a}_{j, k} \partial_{x_{k}} u\right), Q_{\gamma}^{2} X u\right\rangle .
$$


Proposition 4.4. - Suppose that $u \in L^{2}\left([0, T] ; H^{2}\right)$ with $\partial_{t} u \in L^{2}\left([0, T] ; H^{1}\right)$. Then $u_{\gamma}:=Q_{\gamma} u \in C^{0}\left([0, T], H^{1}\right)$ and

$$
\begin{aligned}
& \frac{1}{2} \delta_{0} \delta_{1}\left\|\nabla_{x} u_{\gamma}(t)\right\|_{L^{2}}^{2}+\int_{0}^{t} \delta_{0} \delta_{1}\left\|(\gamma+\lambda \Lambda)^{1 / 2} \nabla_{x} u_{\gamma}\left(t^{\prime}\right)\right\|_{L^{2}}^{2} d t^{\prime} \\
& \leq-2 \operatorname{Re} \int_{0}^{t}\left\langle\widetilde{L}_{2} u, Q_{\gamma}^{2} v\right\rangle d t^{\prime}+C A_{L^{\infty}}^{2}\left\|\nabla_{x} u_{\gamma}(0)\right\|_{L^{2}}^{2}+\int_{0}^{t} E\left(t^{\prime}\right) d t^{\prime}
\end{aligned}
$$

where

$$
\begin{aligned}
|E(t)| \leq & K_{0} A_{L L} A_{L^{\infty}} e^{-2 \gamma t}\left(\|u(t)\|_{H^{1-s(t)+\frac{1}{2} \log }}^{2}+\frac{1}{\delta_{0}^{2}}\|X u(t)\|_{H^{-s(t)+\frac{1}{2} \log }}^{2}\right) \\
& +K e^{-2 \gamma t}\left(\|u(t)\|_{H^{1-s(t)}}^{2}+\|X u(t)\|_{H^{-s(t)}}^{2}\right)
\end{aligned}
$$

To simplify the exposition, we note here that all the dualities $\langle\cdot, \cdot\rangle$ written below make sense, thanks to the smoothness assumption on $u$. This will not be repeated at each step. Moreover, in the proof below, we assume that $u$ itself is smooth (in time).

Proof. - a) We first perform several reductions. Using iii) of Proposition 3.4, one shows that

$$
\left\langle\partial_{x_{j}}\left(\tilde{a}_{j, k} \partial_{x_{k}} u\right), Q_{\gamma}^{2} X u\right\rangle=\left\langle\partial_{x_{j}}\left(T_{\tilde{a}_{j, k}} \partial_{x_{k}} u\right), Q_{\gamma}^{2} X u\right\rangle+E_{1}
$$

with

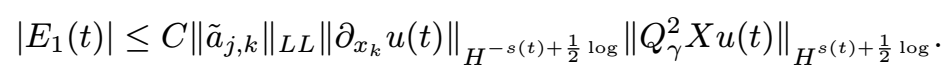

Since $\left\|\tilde{a}_{j, k}\right\|_{L L} \leq K_{0} A_{L L} \leq K_{0} A_{L L} A_{L^{\infty}} / \delta_{0}, E_{1}$ satisfies (4.24). Similarly,

$$
\begin{aligned}
\left\langle\partial_{x_{j}}\left(T_{\tilde{a}_{j, k}} \partial_{x_{k}} u\right), Q_{\gamma}^{2} X u\right\rangle & =\left\langle\partial_{x_{j}} Q_{\gamma} T_{\tilde{a}_{j, k}} \partial_{x_{k}} u, Q_{\gamma} X u\right\rangle \\
& =\left\langle\partial_{x_{j}} T_{\tilde{a}_{j, k}} \partial_{x_{k}} Q_{\gamma} u, Q_{\gamma} X u\right\rangle+E_{2}
\end{aligned}
$$

where $E_{2}$ also satisfies (4.25), and hence (4.24).

b) Next we write

$$
X u=T_{a_{0}} \partial_{t} u+\sum T_{a_{j}} \partial_{x_{j}} u+r
$$

and

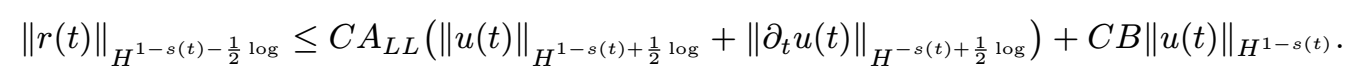

Therefore, $r$ contributes to an error term $E_{3}=\left\langle\partial_{x_{j}} T_{\tilde{a}_{j, k}} \partial_{x_{k}} Q_{\gamma} u, Q_{\gamma} r\right\rangle$ such that

$$
\left|E_{3}(t)\right| \leq e^{-2 \gamma t} K_{0} A_{L^{\infty}}\|u(t)\|_{H^{1-s(t)+\frac{1}{2} \log }}\|r(t)\|_{H^{1-s(t)-\frac{1}{2} \log }} .
$$

Using (4.13) in the estimate of $r$, we see that

$$
\begin{aligned}
\left|E_{3}(t)\right| \leq & e^{-2 \gamma t} K_{0} A_{L^{\infty}} A_{L L}\|u(t)\|_{H^{1-s(t)+\frac{1}{2} \log }} \\
& \left(\|u(t)\|_{H^{1-s(t)+\frac{1}{2} \log }}+\frac{1}{\delta_{0}}\|X u(t)\|_{H^{-s(t)+\frac{1}{2} \log }}+K\|u(t)\|_{H^{1-s(t)}}+K\|X u(t)\|_{H^{-s(t)}}\right)
\end{aligned}
$$

and hence satisfies (4.24). 
c) Consider now the term

$$
\begin{aligned}
\left\langle\partial_{x_{j}} T_{\tilde{a}_{j, k}} \partial_{x_{k}} Q_{\gamma} u, Q_{\gamma} T_{a_{0}} \partial_{t} u\right\rangle & =-\left\langle T_{\tilde{a}_{j, k}} \partial_{x_{k}} Q_{\gamma} u, \partial_{x_{j}} Q_{\gamma} T_{a_{0}} \partial_{t} u\right\rangle \\
& =-\left\langle T_{\tilde{a}_{j, k}} \partial_{x_{k}} Q_{\gamma} u, T_{a_{0}} \partial_{x_{j}} Q_{\gamma} \partial_{t} u\right\rangle+E_{4} \\
& =-\left\langle\left(T_{a_{0}}\right)^{*} T_{\tilde{a}_{j, k}} \partial_{x_{k}} Q_{\gamma} u, \partial_{x_{j}} Q_{\gamma} \partial_{t} u\right\rangle+E_{4} \\
& =-\left\langle T_{a_{0}} T_{\tilde{a}_{j, k}} \partial_{x_{k}} Q_{\gamma} u, \partial_{x_{j}} Q_{\gamma} \partial_{t} u\right\rangle+E_{4}+E_{5} \\
& =-\left\langle T_{a_{0} \tilde{a}_{j, k}} \partial_{x_{k}} Q_{\gamma} u, \partial_{x_{j}} Q_{\gamma} \partial_{t} u\right\rangle+E_{4}+E_{5}+E_{6}
\end{aligned}
$$

where $E_{4}, E_{5}$ and $E_{6}$ are estimated by Proposition 3.7, 3.8 and 3.9 respectively. They all satisfy

$$
\left|E_{k}(t)\right| \leq C e^{-2 \gamma t} A\|u(t)\|_{H^{1-s(t)+\frac{1}{2} \log }}\left\|\partial_{t} u(t)\right\|_{H^{-s(t)+\frac{1}{2} \log }} .
$$

with $A=\left\|\tilde{a}_{j, k}\right\|_{L L}\left\|a_{0}\right\|_{L^{\infty}}+\left\|\tilde{a}_{j, k}\right\|_{L^{\infty}}\left\|a_{0}\right\|_{L L} \leq K_{0} A_{L^{\infty}} A_{L L}$. Again using (4.13) to replace $\partial_{t} u$ by $X u$, one shows that these errors satisfy (4.24).

Similarly

$$
\left\langle\partial_{x_{j}} T_{\tilde{a}_{j, k}} \partial_{x_{k}} Q_{\gamma} u, Q_{\gamma} T_{a_{l}} \partial_{x_{l}} u\right\rangle=-\left\langle T_{a_{l} \tilde{a}_{j, k}} \partial_{x_{k}} Q_{\gamma} u, \partial_{x_{l}} \partial_{x_{j}} Q_{\gamma} u\right\rangle+E_{7}
$$

where $E_{7}$ satisfies

$$
\left|E_{7}(t)\right| \leq C e^{-2 \gamma t} K_{0} A_{L^{\infty}} A_{L L}\|u(t)\|_{H^{1-s(t)+\frac{1}{2} \log }}^{2}
$$

thus (4.24).

d) Introduce the notation

$$
w_{j}=\partial_{x_{j}} Q_{\gamma} u
$$

Because $\tilde{a}_{j, k}=\tilde{a}_{k, j}$, we have

$$
\begin{aligned}
\operatorname{Re}\left\langle T_{a_{l} \tilde{a}_{j, k}} w_{k}, \partial_{x_{l}} w_{j}\right\rangle & +\operatorname{Re}\left\langle T_{a_{l} \tilde{a}_{k, j}} w_{j}, \partial_{x_{l}} w_{k}\right\rangle \\
& =\operatorname{Re}\left\langle\left(\left(T_{a_{l} \tilde{a}_{j, k}}\right)^{*} \partial_{x_{l}}-\partial_{x_{l}} T_{a_{l} \tilde{a}_{j, k}}\right) w_{k}, w_{j}\right\rangle:=E_{8}
\end{aligned}
$$

Using Propositions 3.8 and 3.7, one shows that $E_{8}$ satisfies

$$
\left|E_{8}(t)\right| \leq C\left\|a_{l} \tilde{a}_{j, k}\right\|_{L L}\left\|w_{j}(t)\right\|_{H^{0+\frac{1}{2} \log }}\left\|w_{k}(t)\right\|_{H^{0+\frac{1}{2} \log }}
$$

and therefore $E_{8}$ also satisfies (4.26) thus (4.24).

e) It remains to consider the sum

$$
S:=\operatorname{Re} \sum_{j, k=1}^{n}\left\langle T_{b_{j, k}} \partial_{x_{k}} Q_{\gamma} u, \partial_{x_{j}} Q_{\gamma} \partial_{t} u\right\rangle
$$

with $b_{j, k}=a_{0} \tilde{a}_{j, k}=a_{0} a_{j, k}+a_{j} a_{k}$. By the strict hyperbolicity assumption (2.8), it follows for all $\xi \in \mathbb{R}^{n}$

$$
\sum_{j, k=1}^{n} b_{j, k}(t, x) \xi_{j} \xi_{k} \geq \delta_{0} \delta_{1}|\xi|^{2} .
$$

Therefore, we can use Corollary 3.16. Since $\left\|b_{j, k}\right\|_{L L} \leq 2 A_{L^{\infty}} A_{L L}$, there exists an integer $\nu$, with

$$
\frac{2^{\nu}}{\nu} \approx \frac{A_{L^{\infty}} A_{L L}}{\delta}
$$


such that for all $t \in\left[0, T_{0}\right]$ and $\left(w_{1}, \ldots, w_{n}\right)$ in $L^{2}\left(\mathbb{R}^{n}\right)$, the following estimate is satisfied

$$
\operatorname{Re} \sum_{j, k=1}^{n}\left\langle P_{b_{j, k}(t)}^{\nu} w_{k}, w_{j}\right\rangle \geq \frac{\delta_{0} \delta_{1}}{2}\|w\|_{L^{2}}^{2} .
$$

From now on we fix such a $\nu$ and use the notation $P_{b}$ in place of $P_{b}^{\nu}$.

Using Lemma 3.10 and Proposition 3.14, we see that

$$
\left\|\partial_{x_{j}} T_{b_{j, k}} w_{k}-\partial_{x_{j}} \widetilde{P}_{b_{j, k}} w_{k}\right\|_{H^{0-\frac{1}{2} \log }} \leq C\left\|b_{j, k}\right\|_{L L}\left(\left\|w_{k}\right\|_{H^{0+\frac{1}{2} \log }}+K\left\|w_{k}\right\|_{L^{2}}\right)
$$

Therefore

$$
S=\operatorname{Re} \sum_{j, k=1}^{n}\left\langle\widetilde{P}_{b_{j, k}} \partial_{x_{k}} Q_{\gamma} u, \partial_{x_{j}} Q_{\gamma} \partial_{t} u\right\rangle+E_{9}
$$

where

$$
\begin{aligned}
\left|E_{9}(t)\right| \leq & C e^{-2 \gamma t}\left\|b_{j, k}\right\|_{L L}\|u(t)\|_{H^{1-s(t)+\frac{1}{2} \log }}\left\|\partial_{t} u(t)\right\|_{H^{-s(t)+\frac{1}{2} \log }} \\
& +e^{-2 \gamma t} \nu K\|u(t)\|_{H^{1-s(t)}}\left\|\partial_{t} u(t)\right\|_{H^{-s(t)+\frac{1}{2} \log }} .
\end{aligned}
$$

Using (4.13), implies that $E_{9}$ satisfies (4.24).

Next, we use Proposition 3.14 to replace $\partial_{x_{j}} \widetilde{P}_{b_{j, k}}$ by $\frac{1}{2} \partial_{x_{j}}\left(\widetilde{P}_{b_{j, k}}+\left(\widetilde{P}_{b_{j, k}}\right)^{*}\right)$ at the cost of an error $E_{10}$ similar to $E_{9}$.

At this stage, we commute $Q_{\gamma}$ and $\partial_{t}$ as in (4.19). Using the notation (4.27), yields

$$
\begin{aligned}
2 S= & \sum_{j, k=1}^{n} \operatorname{Re}\left\langle\left(\widetilde{P}_{b_{j, k}}+\left(\widetilde{P}_{b_{j, k}}\right)^{*}\right) w_{k}, \partial_{t} w_{j}\right\rangle \\
& +\gamma \sum_{j, k=1}^{n} \operatorname{Re}\left\langle\left(\widetilde{P}_{b_{j, k}}+\left(\widetilde{P}_{b_{j, k}}\right)^{*}\right) w_{k}, w_{j}\right\rangle \\
& +\lambda \sum_{j, k=1}^{n} \operatorname{Re}\left\langle\left(\widetilde{P}_{b_{j, k}}+\left(\widetilde{P}_{b_{j, k}}\right)^{*}\right) w_{k}, \Lambda w_{j}\right\rangle+2 E_{9}+2 E_{10} .
\end{aligned}
$$

We denote by $S^{1}, S^{2}$ and $S^{3}$ the sums on the right hand side.

f) The symmetry $b_{j, k}=b_{k, j}$ implies the identity

where

$$
S^{1}=\frac{d}{d t} \sum_{j, k=1}^{n} \operatorname{Re}\left\langle\widetilde{P}_{b_{j, k}} w_{k}, w_{j}\right\rangle+E_{11}
$$

is estimated using Proposition 3.14:

$$
\begin{aligned}
\left|E_{11}(t)\right| & \leq C\left\|b_{j, k}\right\|_{L L}\left(\|w(t)\|_{H^{0+\frac{1}{2} \log }}+\nu\|w\|_{L^{2}}\right)\|w(t)\|_{H^{0+\frac{1}{2} \log }} \\
& \leq C e^{-2 \gamma t}\left\|b_{j, k}\right\|_{L L}\|u(t)\|_{H^{1-s(t)+\frac{1}{2} \log }}\left(\|u(t)\|_{H^{1-s(t)+\frac{1}{2} \log }}+\nu\|u(t)\|_{H^{1-s(t)}}\right)
\end{aligned}
$$

and therefore satisfies (4.24). Moreover,

$$
\begin{aligned}
\operatorname{Re}\left\langle\widetilde{P}_{b_{j, k}} w_{k}, \Lambda w_{j}\right\rangle & =\operatorname{Re}\left\langle\widetilde{P}_{b_{j, k}} \Lambda^{\frac{1}{2}} w_{k}, \Lambda^{\frac{1}{2}} w_{j}\right\rangle+\operatorname{Re}\left\langle\Lambda^{\frac{1}{2}}\left[\Lambda^{\frac{1}{2}}, \widetilde{P}_{b_{j, k}},\right] w_{k}, w_{j}\right\rangle \\
\operatorname{Re}\left\langle\left(\widetilde{P}_{b_{j, k}}\right)^{*} w_{k}, \Lambda w_{j}\right\rangle & =\operatorname{Re}\left\langle\Lambda^{\frac{1}{2}} w_{k}, \widetilde{P}_{b_{j, k}} \Lambda^{\frac{1}{2}} w_{j}\right\rangle+\operatorname{Re}\left\langle w_{k},\left[\widetilde{P}_{b_{j, k}}, \Lambda^{\frac{1}{2}}\right] \Lambda^{\frac{1}{2}} w_{j}\right\rangle .
\end{aligned}
$$


We use Proposition 3.17 to estimate the commutators and

$$
S^{3}=2 \sum_{j, k=1}^{n} \operatorname{Re}\left\langle\widetilde{P}_{b_{j, k}} \Lambda^{\frac{1}{2}} w_{k}, \Lambda^{\frac{1}{2}} w_{j}\right\rangle+E_{12}
$$

where $\left|E_{12}(t)\right| \leq K\|w(t)\|_{L^{2}}^{2} \leq K\|u(t)\|_{H^{1-s(t)}}^{2}$.

Summing up, we have shown that up to an error which satisfies (4.24), the quantity (4.22) under consideration is equal to

$$
\begin{aligned}
\frac{d}{d t} \sum_{j, k=1}^{n} \operatorname{Re}\left\langle\widetilde{P}_{b_{j, k}} w_{k}, w_{j}\right\rangle & +\gamma \sum_{j, k=1}^{n} 2 \operatorname{Re}\left\langle\widetilde{P}_{b_{j, k}} w_{k}, w_{j}\right\rangle \\
& +\lambda \sum_{j, k=1}^{n} 2 \operatorname{Re}\left\langle\widetilde{P}_{b_{j, k}} \Lambda^{\frac{1}{2}} w_{k}, \Lambda^{\frac{1}{2}} w_{j}\right\rangle
\end{aligned}
$$

By (4.30), the last two sums are larger than or equal to $\delta_{0} \delta_{1}\|w(t)\|_{L^{2}}^{2}$ and $\delta_{0} \delta_{1}\|w(t)\|_{H^{0+\frac{1}{2} \log }}^{2}$, respectively. Similarly, integrating the first term between 0 and $t$ and using (4.30) give control of $\frac{\delta_{0} \delta_{1}}{2}\|w(t)\|_{L^{2}}$, finishing the proof of (4.23).

\subsection{A-priori estimates for the solutions of (4.11)}

The proof of Theorem 4.1 is based on a-priori estimates for smooth solutions of the system (4.11).

TheOREM 4.5. - There are $\lambda_{0} \geq 0$ of the form (2.30) and $\gamma_{0}$ such that for $\lambda \geq \lambda_{0}$ and $\gamma \geq \gamma_{0}$ the following is true: for all $u \in L^{2}\left([0, T] ; H^{2}\right)$ and $v \in L^{2}\left([0, T] ; H^{1}\right)$ with $\partial_{t} u \in$ $L^{2}\left([0, T] ; H^{1}\right)$ and $\partial_{t} v \in L^{1}\left([0, T] ; L^{2}\right)$ and for all parameters $\lambda, \gamma$ and all $t \leq T$, the following holds:

$$
\begin{aligned}
\sup _{0 \leq t^{\prime} \leq t} e^{-2 \gamma t^{\prime}}( & \left.\frac{1}{2} \delta_{0} \delta_{1}\left\|u\left(t^{\prime}\right)\right\|_{H^{1-s\left(t^{\prime}\right)}}^{2}+\left\|v\left(t^{\prime}\right)\right\|_{H^{-s\left(t^{\prime}\right)}}^{2}\right) \\
& +\delta_{0} \delta_{1} \int_{0}^{t} e^{-2 \gamma t^{\prime}}\left(\lambda\left\|u\left(t^{\prime}\right)\right\|_{H^{1-s\left(t^{\prime}\right)+\frac{1}{2} \log }}^{2}+\gamma\left\|u\left(t^{\prime}\right)\right\|_{H^{1-s\left(t^{\prime}\right)}}^{2}\right) d t^{\prime} \\
& +\int_{0}^{t} e^{-2 \gamma t^{\prime}}\left(\lambda\left\|v\left(t^{\prime}\right)\right\|_{H^{-s\left(t^{\prime}\right)+\frac{1}{2} \log }}^{2}+\gamma\left\|v\left(t^{\prime}\right)\right\|_{H^{-s\left(t^{\prime}\right)}}^{2}\right) d t^{\prime} \\
\leq & C A_{L^{\infty}}^{2}\|u(0)\|_{H^{1-\theta}}^{2}+\|v(0)\|_{H^{-\theta}}^{2}+2 \operatorname{Re} \int_{0}^{t}\left\langle f, Q_{\gamma}^{2} v\right\rangle d t^{\prime} \\
& +K \int_{0}^{t} e^{-2 \gamma t^{\prime}}\left\|g\left(t^{\prime}\right)\right\|_{1-s(t)-\frac{1}{2} \log }\left\|u\left(t^{\prime}\right)\right\|_{1-s(t)+\frac{1}{2} \log } d t^{\prime}
\end{aligned}
$$

with $f=Z v+\tilde{b}_{0} v-\tilde{L}_{2} u+\tilde{L}_{1} u+\tilde{d} u \in L^{1}\left([0, T] ; H^{\alpha^{\prime}-1}\right), g=Y u+\tilde{c}_{0} u-v / a_{0} \in$ $L^{2}\left([0, T] ; H^{\alpha^{\prime}}\right)$ for all $\alpha^{\prime}<\alpha$.

Proof. - We compute the integral over $[0, t]$ of $\operatorname{Re}\left\langle f, Q_{\gamma}^{2} v\right\rangle$. Proposition 4.3 takes care of the first term $2 \operatorname{Re}\left\langle Z v+\tilde{b}_{0} v, Q_{\gamma}^{2} v\right\rangle$. We split the second term into three pieces

$$
\left\langle\tilde{L}_{2} u, Q_{\gamma}^{2} v\right\rangle=\left\langle\tilde{L}_{2} u, Q_{\gamma}^{2} X u\right\rangle-\left\langle\tilde{L}_{2} u, Q_{\gamma}^{2}\left(a_{0} g\right)\right\rangle+\left\langle\tilde{L}_{2} u, Q_{\gamma}^{2}\left(c_{0} u\right)\right\rangle
$$


and use Proposition 4.4 for the first piece. The multiplicative properties imply that

$$
\begin{aligned}
\left|\left\langle\tilde{L}_{2} u(t), Q_{\gamma}^{2}\left(a_{0} g\right)(t)\right\rangle\right| & \leq K\|g(t)\|_{1-s(t)-\frac{1}{2} \log }\left\|\tilde{L}_{2} u(t)\right\|_{-1-s(t)+\frac{1}{2} \log } \\
& \leq K\|g(t)\|_{1-s(t)-\frac{1}{2} \log }\|u(t)\|_{1-s(t)+\frac{1}{2} \log }
\end{aligned}
$$

and

$$
\begin{aligned}
\left|\left\langle\tilde{L}_{2} u(t), Q_{\gamma}^{2}\left(c_{0} u\right)(t)\right\rangle\right| & \leq K\|u(t)\|_{1-s(t)}\left\|\tilde{L}_{2} u(t)\right\|_{-1-s(t)} \\
& \leq K\|u(t)\|_{1-s(t)}^{2} .
\end{aligned}
$$

Next, using the multiplicative properties stated in Corollary 3.6 for the products $\tilde{b}_{j} \partial_{x_{j}} u$ and $\partial_{x_{j}}\left(\tilde{c}_{j} u\right)$, and the embedding $L^{2} \subset H^{-s}$ for the term $\tilde{d} u$, we see that

$$
\left\|\left(\widetilde{L}_{1} u+\tilde{d} u\right)(t)\right\|_{H^{-s(t)}} \leq K\|u(t)\|_{H^{1-s(t)}} .
$$

Thus

$$
\begin{aligned}
\left|\left\langle\left(\tilde{L}_{1}+\tilde{d}\right) u(t), Q_{\gamma}^{2} v(t)\right\rangle\right| & \leq K\|u(t)\|_{1-s(t)}\|v(t)\|_{-s(t)} \\
& \leq K\left(\|u(t)\|_{1-s(t)}^{2}+\|v(t)\|_{-s(t)}^{2}\right) .
\end{aligned}
$$

Proposition 4.4 gives an estimate of $\nabla_{x} u$. We also need an estimate for $u$. The identity (4.20) applied to $u$ yields

$$
\begin{aligned}
e^{-2 \gamma t}\|u(t)\|_{H^{-s(t)}}^{2} & +\int_{0}^{t} e^{-2 \gamma t^{\prime}}\left(\lambda\left\|u\left(t^{\prime}\right)\right\|_{H^{-s\left(t^{\prime}\right)+\frac{1}{2} \log }}^{2}+\gamma\left\|u\left(t^{\prime}\right)\right\|_{H^{-s\left(t^{\prime}\right)}}^{2}\right) d t^{\prime} \\
& =\left\|u_{\gamma}(0)\right\|_{H^{-s(0)}}^{2}+2 \operatorname{Re} \int_{0}^{t}\left\langle\partial_{t} u, Q_{\gamma}^{2} u\right\rangle d t^{\prime}
\end{aligned}
$$

Next, we use the inequality

$$
\left|\left\langle\partial_{t} u, Q_{\gamma}^{2} u\right\rangle\right| \leq C\left(\|u(t)\|_{H^{1-s(t)}}^{2}+\left\|\partial_{t} u(t)\right\|_{H^{-1-s(t)}}^{2}\right) .
$$

In addition, we note that the second equation in (4.11) yields

$$
\left\|\partial_{t} u(t)\right\|_{H^{-1-s(t)}} \leq K\left(\|v(t)\|_{H^{-s(t)}}^{2}+\|u(t)\|_{H^{-s(t)}}^{2}\right)+\|g(t)\|_{H^{-1-s(t)}}^{2} .
$$

We add the various estimates and use Propositions 4.3 and 4.4 to obtain a final estimate. On the left hand side we have

$$
\begin{aligned}
\sup _{0 \leq t^{\prime} \leq t} e^{-2 \gamma t^{\prime}} & \left(\frac{1}{2} \delta_{0} \delta_{1}\left\|u\left(t^{\prime}\right)\right\|_{H^{1-s\left(t^{\prime}\right)}}^{2}+\left\|v\left(t^{\prime}\right)\right\|_{H^{-s\left(t^{\prime}\right)}}^{2}\right) \\
& +\gamma \int_{0}^{t} e^{-2 \gamma t^{\prime}}\left(\delta_{0} \delta_{1}\left\|u\left(t^{\prime}\right)\right\|_{H^{1-s\left(t^{\prime}\right)}}^{2}+\left\|v\left(t^{\prime}\right)\right\|_{H^{-s\left(t^{\prime}\right)}}^{2}\right) d t^{\prime} \\
& +\lambda \int_{0}^{t} e^{-2 \gamma t^{\prime}}\left(\delta_{0} \delta_{1}\left\|u\left(t^{\prime}\right)\right\|_{H^{1-s\left(t^{\prime}\right)+\frac{1}{2} \log }}^{2}+\left\|v\left(t^{\prime}\right)\right\|_{H^{-s\left(t^{\prime}\right)+\frac{1}{2} \log }}^{2}\right) d t^{\prime} .
\end{aligned}
$$

On the right hand side, we find the initial data

$$
C A_{L^{\infty}}^{2}\|u(0)\|_{H^{1-s(0)}}^{2}+\|v(0)\|_{H^{-s(0)}}^{2},
$$

the contribution of $f$

$$
2 \operatorname{Re} \int_{0}^{t}\left\langle f\left(t^{\prime}\right), Q_{\gamma} v\left(t^{\prime}\right)\right\rangle d t^{\prime}
$$


an estimated contribution of $g$

$$
K \int_{0}^{t} e^{-2 \gamma t^{\prime}}\left\|g\left(t^{\prime}\right)\right\|_{1-s\left(t^{\prime}\right)-\frac{1}{2} \log }\left\|u\left(t^{\prime}\right)\right\|_{1-s\left(t^{\prime}\right)+\frac{1}{2} \log } d t^{\prime},
$$

and two types of "remainders":

$$
K_{0} A_{L L} A_{L \infty} \int_{0}^{t} e^{-2 \gamma t^{\prime}}\left(\left\|u\left(t^{\prime}\right)\right\|_{H^{1-s\left(t^{\prime}\right)+\frac{1}{2} \log }}^{2}+\frac{1}{\delta_{0}^{2}}\left\|v_{\gamma}\left(t^{\prime}\right)\right\|_{H^{-s\left(t^{\prime}\right)+\frac{1}{2} \log }}^{2}\right) d t^{\prime}
$$

and

$$
K \int_{0}^{t} e^{-2 \gamma t^{\prime}}\left(\left\|u_{\gamma}\left(t^{\prime}\right)\right\|_{H^{1-s\left(t^{\prime}\right)}}^{2}+\left\|v\left(t^{\prime}\right)\right\|_{H^{-s\left(t^{\prime}\right)}}^{2}\right) d t^{\prime}
$$

If

$$
\lambda \geq 2 K_{0} \frac{A_{L L} A_{L^{\infty}}}{\delta_{0} \delta_{1}} \text { and } \lambda \geq 2 K_{0} \frac{A_{L L} A_{L^{\infty}}}{\delta_{0}^{2}}
$$

the term in (4.40) can be absorbed by (4.36). Note that this choice of $\lambda$ is precisely the choice announced in (2.30), with a new function $K_{0}$ of $A_{L^{\infty}} / \delta_{0}$. Finally, if $\gamma$ is large enough, the term (4.41) is absorbed by (4.35), finishing the proof of the main estimate (4.33).

\subsection{Proof of Theorem 4.1}

From now on, we assume that $\lambda \geq \lambda_{0}$ and $\gamma \geq \gamma_{0}$ are fixed, so that the estimate (4.33) holds. Consider $u, f, u_{0}$ and $u_{1}$ satisfying the equation (4.3) and the smoothness assumptions (4.4), (4.5), (4.6). Consider $v=X u+c_{0} u$, which by Lemma 4.2 satisfies

$$
v \in \mathcal{H}_{-\theta+\frac{1}{2} \log }, \quad \partial_{t} v \in L^{1}\left([0, T] ; H^{-1-\theta_{1}}\right), \quad v_{\mid t=0}=v_{0} \in H^{-\theta},
$$

with $v_{0}=a_{0 \mid t=0} u_{1}+\sum a_{j \mid t=0} \partial_{x_{j}} u_{0}+c_{0 \mid t=0} u_{0}$. In particular, $(u, v, f)$ and $g=0$ satisfy (4.11).

We mollify $u$ and $v$ and introduce, for $\varepsilon>0$,

$$
u_{\varepsilon}=J_{\varepsilon} u, \quad v_{\varepsilon}=J_{\varepsilon} v \quad \text { with } \quad J_{\varepsilon}=\left(1-\varepsilon \Delta_{x}\right)^{-1} .
$$

For all $\varepsilon>0,(4.4)$ and (4.43) imply that

$$
\begin{aligned}
& u_{\varepsilon} \in L^{2}\left([0, T], H^{2}\right), \quad \partial_{t} u_{\varepsilon} \in L^{2}\left([0, T], H^{1}\right), \\
& v_{\varepsilon} \in L^{2}\left([0, T], H^{1}\right), \quad \partial_{t} v_{\varepsilon} \in L^{1}\left([0, T], L^{2}\right),
\end{aligned}
$$

(see (4.7)). Moreover, using the spatial Fourier transform, one immediately sees that $u_{\varepsilon}$ converges to $u$ in $\mathcal{H}_{1-\theta, \lambda}(T)$ and $v_{\varepsilon}$ converges to $v$ in $\mathcal{H}_{-\theta, \lambda}(T)$.

Define

$$
\begin{aligned}
& f_{\varepsilon}=Z v_{\varepsilon}+\tilde{b}_{0} v_{\varepsilon}-\tilde{L}_{2} u_{\varepsilon}+\tilde{L}_{1} u_{\varepsilon}+\tilde{d} u_{\varepsilon} \\
& g_{\varepsilon}=Y u_{\varepsilon}+\tilde{c}_{0} u_{\varepsilon}-v_{\varepsilon} / a_{0}
\end{aligned}
$$

Lemma 4.6. - Assumptions (4.4) and (4.6) imply that $f_{\varepsilon}=f_{1, \varepsilon}+f_{2, \varepsilon}$ with $f_{1, \varepsilon} \rightarrow f_{1}$ in $\mathcal{L}_{-\theta, \lambda}(T)$ and $f_{2, \varepsilon} \rightarrow f_{2}$ in $\mathcal{H}_{-\theta-\frac{1}{2} \log , \lambda}(T)$. Moreover, $g_{\varepsilon} \rightarrow 0$ in $\mathcal{H}_{1-\theta-\frac{1}{2} \log , \lambda}(T)$. 
Taking this lemma for granted, we finish the proof of Theorem 4.1. We use the estimate (4.33) for $\left(u_{\varepsilon}, v_{\varepsilon}\right)$, together with the estimates

$$
\begin{aligned}
\left|\left\langle f_{\varepsilon}(t), Q_{\gamma}^{2} v(t)\right\rangle\right| \leq & C e^{-2 \gamma t}\left(\left\|f_{1, \varepsilon}(t)\right\|_{H^{-s(t)}}\left\|v_{\varepsilon}(t)\right\|_{H^{-s(t)}}\right. \\
& +\left\|f_{2, \varepsilon}(t)\right\|_{\left.H^{-s(t)-\frac{1}{2} \log }\left\|v_{\varepsilon}(t)\right\|_{H^{-s(t)+\frac{1}{2} \log }}\right)}
\end{aligned}
$$

and

$$
\begin{aligned}
& \mid \int_{0}^{t}\left\langle f_{\varepsilon},\right.\left.Q_{\gamma}^{2} v\right\rangle d t^{\prime} \mid \leq C\left(\int_{0}^{t} e^{-\gamma t^{\prime}}\left(\left\|f_{1, \varepsilon}\left(t^{\prime}\right)\right\|_{H^{-s\left(t^{\prime}\right)}} d t^{\prime}\right) \sup _{0 \leq t^{\prime} \leq t} e^{-\gamma t^{\prime}}\left\|v_{\varepsilon}\left(t^{\prime}\right)\right\|_{H^{-s\left(t^{\prime}\right)}}\right. \\
&\left.+C\left(e^{-2 \gamma t^{\prime}}\left\|f_{2, \varepsilon}\left(t^{\prime}\right)\right\|_{H^{-s\left(t^{\prime}\right)-\frac{1}{2} \log }}^{2} d t^{\prime}\right)^{\frac{1}{2}}\left(e^{-2 \gamma t^{\prime}}\left\|v_{\varepsilon}\left(t^{\prime}\right)\right\|_{H^{-s\left(t^{\prime}\right)+\frac{1}{2} \log }}^{2}\right) d t^{\prime}\right)^{\frac{1}{2}} .
\end{aligned}
$$

This implies that there is a $K$ such that for all $\varepsilon>0$, one has

$$
\begin{aligned}
\sup _{0 \leq t^{\prime} \leq t} \| & u_{\varepsilon}\left(t^{\prime}\right)\left\|_{H^{1-s\left(t^{\prime}\right)}}^{2}+\sup _{0 \leq t^{\prime} \leq t}\right\| v_{\varepsilon}\left(t^{\prime}\right) \|_{H^{-s\left(t^{\prime}\right)}}^{2} \\
& +\int_{0}^{t}\left(\left\|u_{\varepsilon}\left(t^{\prime}\right)\right\|_{H^{1-s\left(t^{\prime}\right)+\frac{1}{2} \log }}^{2}+\left\|v_{\varepsilon}\left(t^{\prime}\right)\right\|_{H^{-s\left(t^{\prime}\right)+\frac{1}{2} \log }}^{2}\right) d t^{\prime} \\
\leq & K\left\{\left\|u_{\varepsilon}(0)\right\|_{H^{1-s(0)}}^{2}+\left\|v_{\varepsilon}(0)\right\|_{H^{-s(0)}}^{2}+\int_{0}^{t}\left\|g_{\varepsilon}\left(t^{\prime}\right)\right\|_{H^{1-s(t)-\frac{1}{2} \log }}^{2} d t^{\prime}\right. \\
& \left.+\left(\int_{0}^{t}\left\|f_{1, \varepsilon}\left(t^{\prime}\right)\right\|_{H^{-s\left(t^{\prime}\right)}} d t^{\prime}\right)^{2}+\int_{0}^{t}\left\|f_{2, \varepsilon}\left(t^{\prime}\right)\right\|_{H^{-s\left(t^{\prime}\right)-\frac{1}{2} \log }}^{2} d t^{\prime}\right\} .
\end{aligned}
$$

In addition, there are similar estimates for the differences $\left(u_{\varepsilon}-u_{\varepsilon^{\prime}}, v_{\varepsilon}-v_{\varepsilon^{\prime}}\right)$. Since $u_{\varepsilon}(0)=$ $J_{\varepsilon} u_{0}$ and $v_{\varepsilon}(0)=J_{\varepsilon} v_{0}$ converge to $u_{0}$ and $v_{0}$ in $H^{1-s(0)}$ and $H^{-s(0)}$, respectively, the estimate implies that $u_{\varepsilon}$ is a Cauchy sequence in $\mathcal{H}_{1-\theta, \lambda}(T)$ and in $C^{0}\left([0, t] ; H^{1-s(t)}\right)$ for all $t \in[0, T]$. Therefore, the limit $u$ in $\mathcal{H}_{1-\theta, \lambda}(T)$ also belongs to $\mathcal{C}_{1-\theta, \lambda}(T)$. Similarly, $v_{\varepsilon}$ is a Cauchy sequence in $\mathcal{H}_{-\theta, \lambda}(T)$ and in $C^{0}\left([0, t] ; H^{-s(t)}\right)$ for all $t \in[0, T]$ and $v \in \mathcal{C}_{1-\theta, \lambda}(T)$. In addition, we can pass to the limit in (4.45) proving that

$$
\begin{aligned}
\sup _{0 \leq t^{\prime} \leq t}\left\|u\left(t^{\prime}\right)\right\|_{H^{1-s\left(t^{\prime}\right)}}^{2}+\sup _{0 \leq t^{\prime} \leq t}\left\|v\left(t^{\prime}\right)\right\|_{H^{-s\left(t^{\prime}\right)}}^{2} & \\
& +\int_{0}^{t}\left(\left\|u\left(t^{\prime}\right)\right\|_{H^{1-s\left(t^{\prime}\right)+\frac{1}{2} \log }}^{2}+\left\|v\left(t^{\prime}\right)\right\|_{H^{-s\left(t^{\prime}\right)+\frac{1}{2} \log }}^{2}\right) d t^{\prime} \\
\leq & K\left\{\left\|u_{0}\right\|_{H^{1-s(0)}}^{2}+\left\|v_{0}\right\|_{H^{-s(0)}}^{2}\right. \\
& \left.+\left(\int_{0}^{t}\left\|f_{1}\left(t^{\prime}\right)\right\|_{H^{-s\left(t^{\prime}\right)}} d t^{\prime}\right)^{2}+\int_{0}^{t}\left\|f_{2}\left(t^{\prime}\right)\right\|_{H^{-s\left(t^{\prime}\right)-\frac{1}{2} \log }}^{2} d t^{\prime}\right\} .
\end{aligned}
$$

Using the equation $Y u+\tilde{c}_{0} u=v / a_{0}$ and the estimate (4.13) of Lemma 4.2 to bound the time derivative $\partial_{t} u$, we see that $\partial_{t} u \in \mathcal{C}_{-\theta, \lambda}(T)$ and that the energy estimate (2.29) is satisfied.

Therefore, it remains only to prove the lemma.

Proof of Lemma 4.6. - By assumption (4.6), $f=f_{1}+f_{2}$ and $J_{\varepsilon} f_{1} \rightarrow f_{1}$ in $\mathcal{L}_{-\theta, \lambda}(T)$ and $J_{\varepsilon} f_{2} \rightarrow f_{2}$ in $\mathcal{H}_{-\theta-\frac{1}{2} \log , \lambda}(T)$. Therefore, it is sufficient to prove that the commutators

$$
\begin{aligned}
& {\left[Z, J_{\varepsilon}\right] v, \quad\left[\tilde{L}_{2}, J_{\varepsilon}\right] u,} \\
& {\left[\tilde{b}_{0}, J_{\varepsilon}\right] v, \quad\left[\tilde{L}_{1}, J_{\varepsilon}\right] u, \quad\left[\tilde{d}, J_{\varepsilon}\right] u,}
\end{aligned}
$$


converge to 0 in $\mathcal{H}_{-\theta, \lambda}(T)$ and that the commutators

$$
\left[Y, J_{\varepsilon}\right] u, \quad\left[\tilde{c}_{0}, J_{\varepsilon}\right] u, \quad\left[1 / a_{0}, J_{\varepsilon}\right] v
$$

converge to 0 in $\mathcal{H}_{1-\theta, \lambda}(T)$. We note that $J_{\varepsilon}$ commutes with $\partial_{t}$ in $Z$ and $Y$. Thanks to (4.4) (4.43) and to the conservative form of $Z$ and $\tilde{L} *_{2}$, we see that there are four types of commutators to consider :

(4.47) $\left[a, J_{\varepsilon}\right] w \rightarrow 0$ in $\mathcal{H}_{1-\theta, \lambda}(T), \quad$ when $a \in L^{\infty} \cap L L\left([0, T] \times \mathbb{R}^{d}\right), \quad w \in \mathcal{H}_{-\theta, \lambda}(T)$,

$$
\begin{aligned}
{\left[b, J_{\varepsilon}\right] w } & \rightarrow 0 \text { in } \mathcal{H}_{-\theta, \lambda}(T), \\
{\left[c, J_{\varepsilon}\right] w } & \rightarrow 0 \text { in } \mathcal{H}_{1-\theta, \lambda}(T), \quad \text { when } b \in C^{\alpha}\left([0, T] \times \mathbb{R}^{d}\right), w \in \mathcal{H}_{-\theta, \lambda}\left([0, T] \times \mathbb{R}^{d}\right), w \in \mathcal{H}_{1-\theta, \lambda}(T), \\
{\left[d, J_{\varepsilon}\right] w } & \rightarrow 0 \text { in } \mathcal{H}_{-\theta, \lambda}(T), \quad \text { when } d \in L^{\infty}\left([0, T] \times \mathbb{R}^{d}\right), w \in \mathcal{H}_{1-\theta, \lambda}(T)
\end{aligned}
$$

The first commutators $\left[a, J_{\varepsilon}\right]=\left[T_{a}, J_{\varepsilon}\right]+R_{a}, J_{\varepsilon}-J_{\varepsilon} R_{a}$ are uniformly bounded from $\mathcal{H}_{-\theta, \lambda}(T)$ to $\mathcal{H}_{1-\theta, \lambda}(T)$ : this is true for the first term by Proposition 3.7, since the $J_{\varepsilon}$ form a bounded family of operators of degree 0 ; for the last two terms, this follows from Proposition 3.4. Moreover, $\left[a, J_{\varepsilon}\right] w \rightarrow 0$ in $L^{2}\left([0, T] ; H^{\sigma}\right)$ for all $\sigma<1$, and thus also in $\mathcal{H}_{1-\theta, \lambda}$, when $w$ is smooth and $a \in L^{\infty} \cap L L$. By density, this implies (4.47).

For the commutators (4.48), we note that they are uniformly bounded from $\mathcal{H}_{-\theta, \lambda}(T)$ to $\mathcal{H}_{-\theta, \lambda}(T)$. This is true for both terms $b J_{\varepsilon}$ and $J_{\varepsilon} b$ since $s(t)$ remains in a compact subset of $\left[0, \alpha\left[\right.\right.$. Because $\left[b, J_{\varepsilon}\right] w$ converges to zero in $L^{2}\left([0, T] ; H^{\sigma}\right)$ for all $\sigma<\alpha$, when $w$ is smooth and $b \in L^{\infty} \cap L L$, the convergence in (4.48) follows. The proof for (4.49) is similar.

Finally, we note that $\left[d, J_{\varepsilon}\right] w \rightarrow 0$ in $L^{2}\left([0, T] \times \mathbb{R}^{d}\right)$, hence in $\mathcal{H}_{-\theta, \lambda}(T)$ when $d \in L^{\infty}\left([0, T] \times \mathbb{R}^{d}\right)$ and $w \in L^{2}\left([0, T] \times \mathbb{R}^{d}\right)$, thus in particular when $w \in \mathcal{H}_{1-\theta, \lambda}(T)$.

\subsection{Existence and uniqueness}

Proof of Theorem 2.4. - Assume that $u \in H^{s}(] 0, T\left[\times \mathbb{R}^{n}\right)$ with $\left.s \in\right] 1-\alpha, \alpha\left[, T \leq T_{0}\right.$, and satisfies

$$
L u=0, \quad u_{\mid t=0}=0, \quad X u_{\mid t=0}=0 .
$$

We want to prove that $u=0$.

Fix $\theta<\theta_{1}$ in $] 1-\alpha, \alpha\left[\right.$ with $1-\theta<s$. Let $\lambda$ and $T^{\prime}$ be the parameter and time associated to them by Theorem 4.1. Note that they depend only on $\theta, \theta_{1}$, the norms $A_{L^{\infty}}$ and $A_{L L}$ in (2.9) and the constants of hyperbolicity $\delta_{0}$ and $\delta_{1}$ in (2.8).

From Lemma 2.2, we know that $u \in L^{2}\left([0, T] ; H^{s}\left(\mathbb{R}^{n}\right)\right)$ and $\partial_{t} u \in L^{2}\left([0, T] ; H^{s-1}\left(\mathbb{R}^{n}\right)\right)$ and therefore, on $\left[0, T^{\prime}\right] \times \mathbb{R}^{n}, u \in \mathcal{H}_{1-\theta+\frac{1}{2} \log , \lambda}$ and $\partial_{t} u \in \mathcal{H}_{-\theta+\frac{1}{2} \log , \lambda}$ since $s>1-\theta-\lambda t$. By Theorem 4.1, $u$ satisfies the energy estimate (2.29) on $\left[0, T^{\prime}\right]$, and since the right hand side vanishes, $u=0$ for $t<T^{\prime}$. By a finite number of iterations, $u$ vanishes for $t<T$.

Proof of Theorem 2.6. - On $\left[0, T_{0}\right] \times \mathbb{R}^{d}$, the coefficients of $L_{2}$ can be approximated in $L^{\infty}$ and $C^{\alpha^{\prime}}$ for all $\alpha^{\prime}<1$ by $C^{\infty}$ functions which are uniformly bounded in $L^{\infty}$ and in $L L$, in such a way that the hyperbolicity condition (2.8) remains satisfied. Similarly, the coeffficients of $L_{1}$ can be approximated in $L^{\infty}$ and $C^{\alpha^{\prime}}$ for all $\alpha^{\prime}<\alpha$ by smooth functions which are uniformly bounded in $C^{\alpha}$. Further, the coefficient $c$ can be approximated in $L_{l o c}^{2}$ by functions uniformly bounded in $L^{\infty}$. This defines operators $L^{\varepsilon}$ with $C^{\infty}$ coefficients which satisfy (2.8), 
(2.9) and (2.10) uniformly in $\varepsilon$ and converge to the coefficients of $L$ in the sense described above.

We fix the parameter $\lambda \geq \lambda_{0}$, where $\lambda_{0}$ is given by Theorem 4.1. Recall that $T$ is then given by (4.2). Consider Cauchy data $u_{0} \in H^{1-\theta}$ and $u_{1} \in H^{-\theta}$ and a source term $f=f_{1}+f_{2}$ with $f_{1} \in \mathcal{L}_{-\theta, \lambda}(T)$ and $f_{2} \in \mathcal{H}_{-\theta-\frac{1}{2} \log , \lambda}$. We can approximate these data in the corresponding spaces by $C^{\infty}$ functions $u_{0}^{\varepsilon}, u_{1}^{\varepsilon}, f_{1}^{\varepsilon}$ and $f_{2}^{\varepsilon}$, compactly supported in $x$. The strictly hyperbolic problems with smooth coefficients and smooth data

$$
L^{\varepsilon} u^{\varepsilon}=f_{1}^{\varepsilon}+f_{2}^{\varepsilon}, \quad u_{\mid t=0}^{\varepsilon}=u_{0}^{\varepsilon}, \quad X^{\varepsilon} u_{\mid t=0}^{\varepsilon}=u_{1}^{\varepsilon}
$$

have a unique smooth solution $u^{\varepsilon}$, compactly supported in $x$.

By Theorem 4.1, the energy estimate (2.29) is satisfied with a constant $K$ independent of $\varepsilon$. Therefore the family $\left\{u^{\varepsilon}\right\}$ is bounded in $\mathcal{H}_{1-\theta+\frac{1}{2} \log , \lambda}$, thus in $L^{2}\left([0, T], H^{1-\theta_{1}}\right)$ and the families $\left\{\partial_{t} u^{\varepsilon}\right\}$ and $\left\{X^{\varepsilon} u^{\varepsilon}\right\}$ are bounded in $\mathcal{H}_{-\theta+\frac{1}{2} \log , \lambda}$, hence in $L^{2}\left([0, T], H^{-\theta_{1}}\right)$. Therefore, extracting a subsequence if necessary, $u^{\varepsilon}$ converges to a limit $u$, weakly in $L^{2}\left([0, T], H^{1-\theta_{1}}\right)$ and in $H^{1}\left([0, T], H^{-\theta_{1}}\right)$. Moreover, $u \in \mathcal{H}_{1-\theta+\frac{1}{2} \log , \lambda}$ and $\partial_{t} u \in \mathcal{H}_{-\theta+\frac{1}{2} \log , \lambda}$. There is no difficulty in passing to the limit in the equation in the sense of distributions: all the products are well defined and involve one strong and one weak convergence. Thus $L u=f$.

The weak convergence in $L^{2}\left([0, T], H^{1-\theta_{1}}\right) \cap H^{1}\left([0, T], H^{-\theta_{1}}\right)$ implies the strong convergence in $C^{0}\left([0, T] ; H_{l o c}^{-\theta_{1}}\right)$ and therefore the convergence of $u_{\mid t=0}^{\varepsilon}$ to $u_{\mid t=0}$ in $H_{l o c}^{-\theta_{1}}$. Therefore, $u_{\mid t=0}=u_{0}$.

Using the equation as in Lemma 2.2, we prove that the family $v^{\varepsilon}=X^{\varepsilon} u^{\varepsilon}+c_{0}^{\varepsilon} u^{\varepsilon}$, which converges weakly to $v=X u+c_{0} u$, is bounded in $L^{2}\left([0, T], H^{-\theta_{1}}\right) \cap H^{1}\left([0, T], H^{-1-\theta_{1}}\right)$. Thus $v_{\mid t=0}^{\varepsilon}$ converges to $v_{\mid t=0}$ in $H_{l o c}^{-\theta_{1}}$. Hence $v_{\mid t=0}=u_{1}+c_{0 \mid t=0} u_{0}$ implying that $X u_{\mid t=0}=$ $u_{1}$.

By Theorem 4.1 the solution $u$ also belongs to $\mathcal{C}_{1-\theta, \lambda}$ with $\partial_{t} u \in \mathcal{C}_{1-\theta, \lambda}$ and satisfies the energy estimate (2.29).

\section{Local results}

We consider the equation (1.1) together with an initial hypersurface $\Sigma$ satisfying Assumption 1.1. This section contains the proofs of Proposition 1.4 and Theorems 1.5 and 1.6.

\subsection{Change of coordinates. Traces}

Consider a smooth change of variables $y=\chi(\tilde{y})$ and for a function $u$ let $\tilde{u}$ denote $u \circ \chi$. Then

$$
\left(\partial_{y_{j}} u\right) \circ \chi=\sum_{k} \psi_{j, k}^{\prime} \partial_{\tilde{y}_{k}} \tilde{u}=\sum_{k} \partial_{\tilde{y}_{k}}\left(\psi_{j, k}^{\prime} \tilde{u}\right)-\left(\sum_{k} \partial_{\tilde{y}_{k}} \psi_{j, k}^{\prime}\right) \tilde{u}
$$

with $\left.\psi_{j, k}^{\prime}=\left(\partial_{y_{j}} \psi_{k}\right) \circ \chi\right)$ and $\psi=\chi^{-1}$. Thus

$$
\widetilde{L u}=\widetilde{L} \tilde{u}
$$

where $\widetilde{L}$ has the same form as $L$ and satisfies Assumption 1.1. 
If $\nu(y)$ is conormal to $\Sigma$, then $\tilde{\nu}(\tilde{y})={ }^{t} \chi^{\prime}(y) \nu(\chi(\tilde{y}))$ is conormal to $\widetilde{\Sigma}=\chi^{-1}(\Sigma)$. Using the notations (1.7), for smooth functions, the Neumann traces associated to $(L, \nu)$ and $(\widetilde{L}, \tilde{\nu})$, are linked by the relation

$$
\left(N_{\nu} u\right) \circ \chi=\widetilde{N}_{\tilde{\nu}} \tilde{u}
$$

The Green's formula (1.9) can be transported by $\chi$, taking into account the Jacobian factors:

$$
(f, g)_{L^{2}\left(\Omega_{+}\right)}=(\tilde{f}, J \tilde{g})_{L^{2}\left(\widetilde{\Omega}_{+}\right)}
$$

with $J=\left|\operatorname{det} \chi^{\prime}\right|$. This relation extends to the duality $H^{s} \times H^{-s}$ for $|s|<\frac{1}{2}$. In particular, comparing the Green formula for $L$ and $\widetilde{L}$ tested on smooth functions implies that :

$$
\begin{aligned}
(\widetilde{L})^{*}(J \tilde{v}) & =J \widetilde{L^{*} v} \\
\widetilde{N}_{\tilde{\nu}}^{\prime}(J \tilde{v}) & =J_{\Sigma} \widetilde{N_{\nu}^{\prime} v}
\end{aligned}
$$

where $J_{\Sigma}$ is the Jacobian of $\chi_{\mid \widetilde{\Sigma}}$.

As a corollary, the statement of Proposition 1.4 is invariant by smooth changes of variables and therefore can be proved in any suitable system of coordinates.

Proof of Proposition 1.4. - a) Uniqueness. We prove that if $u_{0} \in H_{l o c}^{s-\frac{1}{2}}$ and $u_{1} \in H_{l o c}^{s-\frac{3}{2}}$ satisfy

$$
\left\langle u_{1}, D_{\Sigma} v\right\rangle_{H^{s-\frac{3}{2}} \times H^{\frac{3}{2}-s}}-\left\langle u_{0}, N_{\Sigma}^{\prime} v\right\rangle_{H^{s-\frac{1}{2}} \times H^{\frac{1}{2}-s}}=0
$$

for all $\left.s^{\prime} \in\right] 1-\alpha, \frac{1}{2}\left[\right.$ such that $s^{\prime} \leq s$ and all $v \in H_{\text {comp }}^{2-s^{\prime}}(\Omega \cap\{\varphi \geq 0\})$, then $u_{0}=u_{1}=0$.

It is sufficient to prove that for $v_{0}$ and $v_{1}$ in $C_{0}^{\infty}(\Omega \cap \Sigma)$, there is $v \in C_{0}^{1+\alpha}(\Omega)$ such that $v_{\mid \Sigma}=v_{0}$ and $N_{\nu}^{\prime} v=v_{1}$. This can be done in local coordinates $y=(t, x)$ where $\Sigma=\{t=0\}$ and this amounts to solve

$$
v_{\mid t=0}=v_{0}, \quad \partial_{t} v_{\mid t=0}=g_{0} v_{1}+\sum g_{j} \partial_{x_{j}} v_{0}+h v_{0}
$$

where $g_{0}, \ldots, g_{d}$ belong to $L L(\Omega)$ and $h$ belongs to $C^{\alpha}(\Omega)$.

b) Existence. According to the discussion above we may assume that we are working in coordinates $y=(t, x)$ such that $\underline{y}=(0,0)$ and $\Sigma=\{t=0\}$. The conormal direction is $\nu=\lambda(x) d t$ and the Neumann trace for smooth functions is:

$$
N_{\nu} u=\lambda(X u)_{\mid t=0}
$$

where $X$ is the vector field (2.11).

Let $s \in] 1-\alpha, \alpha\left[\right.$. For $u \in H_{l o c}^{s}(\Omega \cap\{t \geq 0\})$ such that $L u \in L^{2}\left(\Omega_{+}\right)$. Local versions of Lemmas 2.1 and 2.2 imply that for $T>0$ small and $\omega$ relatively compact in $\Omega \cap\{t=0\}$

$$
\left.\left.u \in H^{1, s-1}(] 0, T[\times \omega)\right), \quad X u \in H^{1, s-2}(] 0, T[\times \omega)\right) .
$$

Indeed, the proofs are identical, using local multiplicative properties and local versions of the spaces $H^{s, s^{\prime}}$. The trace operator $w \mapsto w_{\mid t=0}$ has a unique extension as a bounded operator from $H^{1, \sigma}(] 0, T[\times \omega)$ to $H^{\sigma+\frac{1}{2}}(\omega)$. Therefore, the traces $u_{\mid t=0}$ and $X u_{\mid t=0}$ are well defined in $H^{s-\frac{1}{2}}$ and $H^{s-\frac{3}{2}}$ respectively. We show that, in these coordinates, Green's formula (1.9) holds with

$$
D_{\Sigma} u=u_{\mid t=0}, \quad N_{\nu} u=\lambda(X u)_{\mid t=0} .
$$


This follows immediately by integration by parts, the only difficulty is to check that at each step we have enough smoothness to justify the computations. We sketch here the main points of the discussion. First, recall that for $w \in H^{1, \sigma}$ and $v \in H^{1,-1-\sigma}$ compactly supported in $[0, T[\times \omega:$

$$
\left\langle\partial_{t} w, v\right\rangle_{L^{2}(\sigma)}=-\left\langle w, \partial_{t} v\right\rangle_{L^{2}(\sigma+1)}+\left\langle w_{\mid t=0}, v_{\mid t=0}\right\rangle_{H^{\sigma+\frac{1}{2}} \times H^{-\frac{1}{2}-\sigma}}
$$

and for $w \in H^{0, \sigma+1}$ and $v \in H^{0,-\sigma}$ compactly supported in $[0, T[\times \omega$ :

$$
\left\langle\partial_{x_{j}} w, v\right\rangle_{L^{2}(\sigma)}=-\left\langle w, \partial_{x_{j}} v\right\rangle_{L^{2}(\sigma+1)}
$$

where $\langle\cdot, \cdot\rangle_{L^{2}(\sigma)}$ denotes the duality $L^{2}\left([0, T], H^{\sigma}\right) \times L^{2}\left([0, T] ; H^{-\sigma}\right)$ and the traces are taken in the sense indicated above.

Consider $v \in H_{\text {comp }}^{2-s^{\prime}}\left(\left[0, T[\times \omega)\right.\right.$, where $\left.s^{\prime} \in\right] 1-\alpha, \frac{1}{2}\left[\right.$ with $s^{\prime} \leq s$. Using (5.6), (5.8), (5.9) and the multiplicative properties of functions in LL-functions and $H^{\sigma}$, one obtains that

$$
\begin{aligned}
\langle Z X u, v\rangle_{L^{2}(s-2)} & =\left\langle X u, Z^{*} v\right\rangle_{L^{2}(s-1)}+\left\langle X u_{\mid t=0}, v_{\mid t=0}\right\rangle_{H^{s-\frac{3}{2}} \times H^{\frac{3}{2}-s}}, \\
& =\left\langle Y u, \bar{a}_{0} Z^{*} v\right\rangle_{L^{2}(s-1)}+\left\langle X u_{\mid t=0}, v_{\mid t=0}\right\rangle_{H^{s-\frac{3}{2}} \times H^{\frac{3}{2}-s}},
\end{aligned}
$$

(recall the definitions (2.11) and (2.13) of $X, Y$ and $Z$ ). Let $w=\bar{a}_{0} Z^{*} v \in H^{1-s^{\prime}}$. Because $1-s^{\prime} \geq 0$ and $s^{\prime} \leq s, w \in H^{0,1-s^{\prime}} \subset H^{0,1-s}$. Therefore,

$$
\left\langle\tilde{a}_{j} \partial_{x_{j}} u, w\right\rangle_{L^{2}(s-1)}=-\left\langle u, \partial_{x_{j}}\left(\overline{\tilde{a}_{j}} v\right)\right\rangle_{L^{2}(s)} .
$$

The term $\left\langle\partial_{t} u, w\right\rangle$ is more delicate since $\partial_{t} w \in H^{-s^{\prime}}$ and $s^{\prime}>0$. However, as in Lemma 1.3, one can use the duality $H^{s^{\prime}}(\{t \geq 0\}) \times H^{-s^{\prime}}(\{t \geq 0\})$ for $0 \leq s^{\prime}<\frac{1}{2}$ and for $u \in H^{1, s-1}$ and $w \in H_{\text {comp }}^{1-s^{\prime}}$, (5.8) can be extended as

$$
\left\langle\partial_{t} u, w\right\rangle_{L^{2}(s-1)}=-\left\langle u, \partial_{t} w\right\rangle_{H^{s^{\prime}} \times H^{-s^{\prime}}}+\left\langle u_{\mid t=0}, w_{\mid t=0}\right\rangle_{H^{s+\frac{1}{2}} \times H^{\frac{1}{2}-s}},
$$

noticing that the trace $w_{\mid t=0}$ belongs to $H^{\frac{1}{2}-s^{\prime}} \subset H^{\frac{1}{2}-s}$.

Repeated use of (5.9) implies that for the tangential second order part $\widetilde{L}_{2}$ defined in (2.13), there holds

$$
\left\langle\widetilde{L}_{2} u, v\right\rangle_{L^{2}(s-2)}=\left\langle u,\left(\widetilde{L}_{2}\right)^{*} v\right\rangle_{L^{2}(s)} .
$$

First order terms are treated similarly, and summing up we get that

$$
\begin{aligned}
& (L u, v)_{L^{2}(\{t>0\}}-\left\langle u, L^{*} v\right\rangle_{H^{s^{\prime}}(\{t>0\}) \times H^{-s^{\prime}}(\{t>0\})} \\
& \quad=\left\langle X u_{\mid t=0}, v_{\mid t=0}\right\rangle_{H^{s-\frac{3}{2}} \times H^{\frac{3}{2}-s}}-\left\langle u_{\mid t=0}, X^{\prime} v_{\mid t=0}\right\rangle_{H^{s-\frac{1}{2}} \times H^{\frac{1}{2}-s}}
\end{aligned}
$$

In the computations above, the underlying measure in $\{t=0\}$ is the Lebesque measure $d x$. The surface measure associated to the conormal $\lambda d t$ as in (1.6) is $\lambda^{-1} d x$. This proves that the identity (1.6) is proved with $D_{\Sigma}$ and $N_{\nu}$ given by (5.7), as claimed. 


\subsection{Local existence}

Choose $\Phi$, a smooth map from $\mathbb{R}^{1+n}$ to $\Omega$, with $\Phi(y)=y$ on a smaller neighborhood $\Omega_{1}$ and $\Phi(y)=0$ for $y$ large enough. Changing the coefficients according to the rule $a^{\sharp}(y)=$ $a(\Phi(y))$ we obtain an operator $L^{\sharp}$ which coincides with $L$ on $\Omega_{1}$, satisfies the regularity conditions (2.4) to (2.6), and the hyperbolicity conditions (2.8) globally on $\mathbb{R}^{1+n}$.

Fix $s>1-\alpha$. Without loss of generality for the statement of Theorem 1.5, we can assume that $s<\alpha$. We are going to apply Theorem 2.6 to the operator $L^{\sharp}$ with $\left.\theta=1-s \in\right] 1-\alpha, \alpha[$. Choosing $\left.\theta_{1} \in\right] \theta, \alpha\left[\right.$, this theorem provides us with $\lambda$ and $T=\left(\theta_{1}-\theta\right) / \lambda$. We fix $\Omega^{\prime}=$ $\Omega_{1} \cap\{|t|<T\}$.

Suppose that $u_{0}$ and $u_{1}$ are Cauchy data in $H^{s}(\omega)$ and $H^{s-1}(\omega)$ respectively, on a neighborhood $\omega$ of 0 in $\mathbb{R}^{n}$. There are restrictions to $\omega$ of functions $u_{0}^{\sharp} \in H^{s}\left(\mathbb{R}^{n}\right)$ and $u_{1}^{\sharp} \in$ $H^{s-1}\left(\mathbb{R}^{n}\right)$ respectively. Suppose that $f \in L^{2}\left(\Omega^{\prime} \cap\{t>0\}\right)$. We extend it, for instance by 0 , to $f^{\sharp} \in L^{2}\left([0, T] \times \mathbb{R}^{n}\right)$. By Theorem 2.6, the Cauchy problem

$$
L^{\sharp} u^{\sharp}=f^{\sharp}, \quad u_{\mid t=0}^{\sharp}=u_{0}^{\sharp}, \quad\left(X^{\sharp} u^{\sharp}\right)_{\mid t=0}=u_{1}^{\sharp}
$$

has a solution $u^{\sharp}$ on $[0, T] \times \mathbb{R}^{n}$, which belongs in particular to $L^{2}\left([0, T] ; H^{s_{1}}\right)$ with $s_{1}=$ $1-\theta_{1}$ and such that $\partial_{t} u \in L^{2}\left([0, T] ; H^{s_{1}-1}\right)$. In particular, $u^{\sharp} \in H^{s_{1}}\left([0, T] \times \mathbb{R}^{n}\right)$ and by restriction to $\Omega^{\prime}$ defines a solution of (1.10).

\subsection{Local uniqueness}

To prove Theorem 1.6, we first reduce the problem to proving a theorem of propagation of zero across the surface $\{t=0\}$.

Lemma 5.1. - Suppose that $s>1-\alpha$ and $u \in H^{s}(\Omega \cap\{t>0\})$ satisfies

$$
L u=0, \quad u_{\mid t=0}=0, \quad X u_{\mid t=0}=0 .
$$

Then the extension $u_{e}$ of $u$ by 0 for $t<0$ satisfies

$$
u_{e} \in H^{s} \text { and } L u_{e}=0
$$

on a neighborhood $\Omega_{1}$ of 0 .

Proof. - If the coefficients were smooth, this would be immediate. We check that we have enough smoothness to extend the result to our case.

We can assume that $\Omega=]-T, T[\times \omega$. From Lemma 2.2 (localized in space) we know that $u \in L^{2}\left([0, T] ; H_{l o c}^{s}(\omega)\right)$, thus its extension $u_{e} \in L^{2}\left([-T, T] ; H_{l o c}^{s}(\omega)\right)$. Moreover, $\partial_{t} u \in$ $L^{2}\left([0, T] ; H_{l o c}^{s-1}(\omega)\right)$ and by assumption $u_{\mid t=0}=0$. Therefore, $\partial_{t} u_{e}$ is the extension of $\partial_{t} u$ by 0 and thus belongs to $L^{2}\left([-T, T] ; H_{l o c}^{s-1}(\omega)\right)$. In particular, $u_{e} \in H_{l o c}^{s}(]-T, T[\times \omega)$.

Let $v=X u+c_{0} u \in L^{2}\left([0, T] ; H_{l o c}^{s-1}(\omega)\right)$ and let $v_{e} \in L^{2}\left([-T, T] ; H_{l o c}^{s-1}(\omega)\right)$ denote its extension by 0 . The first step implies that $X u_{e}$ is the extension of $X u$ and therefore $v_{e}=$ $X u_{e}+c_{0} u_{e}$. Write the equation as

$$
\partial_{t} v=P(u, v)
$$

where $P$ involves only spatial derivatives (see (2.17)). Morever, we have seen in the proof of Lemma 2.2 that $P(u, v) \in L^{2}\left([0, T] ; H_{l o c}^{s-2}(\omega)\right)$. Since by assumption the trace of $v$ vanishes, this implies that $\partial_{t} v_{e}$ is the extension by 0 of $\partial_{t} v$, thus the extension of $P(u, v)$, that 
is $P\left(u_{e}, v_{e}\right)$. Since $v_{e}=X u_{e}+c_{0} u_{e}$, this means that $u_{e}$ satisfies the equation on $\Omega=$ ]$-T, T[\times \omega$.

We now finish the proof of Theorem 1.6. We suppose that $u \in H^{s}(\Omega \cap\{t>0\})$ satisfies (5.13), with $s>1-\alpha$ and we denote by $u_{e}$ its extension by 0 for $t<0$. We use the classical convexification method, and consider the change of variables

$$
(t, x) \mapsto(\tilde{t}, \tilde{x}) \quad \tilde{t}=t+|x|^{2}, \tilde{x}=x,
$$

which maps the past $\{t<0\}$ to $\left\{\tilde{t}<|\tilde{x}|^{2}\right\}$. Thus there is $T_{0}>0$ such that the function $\tilde{u}$ deduced from $u_{e}$ is defined for $\tilde{t}<T_{0}$ and vanishes for $\tilde{t}<|\tilde{x}|^{2}$. Moreover, decreasing $T_{0}$ if necessary, the operator $\tilde{L}$ deduced from $L$ is defined on a neighborhood $\tilde{\Omega}$ of the origin which contains the closed lens $\bar{D}=\left\{|\tilde{x}|^{2} \leq t \leq T_{0}\right\}$ and $\tilde{L} \tilde{u}=0$ on $\tilde{\Omega} \cap\left\{t<T_{0}\right\}$. Now we extend the coefficients of $\tilde{L}$, as above, and obtain a new operator $L^{\sharp}$, defined on $\mathbb{R}^{1+n}$, satisfying the assumptions of section 2 , and equal to $\tilde{L}$ on a neighborhood of $\bar{D}$. Therefore, on $]-\infty, T_{0}\left[\times \mathbb{R}^{n}\right.$

$$
L^{\sharp} \tilde{u}=0, \quad \tilde{u} \in H^{s}, \quad \tilde{u}_{\mid\left\{\tilde{t}<|\tilde{x}|^{2}\right\}}=0 .
$$

Since $\tilde{u}$ vanishes in the past, the traces $\tilde{u}_{\mid t=-\varepsilon}$ and $X^{\sharp} \tilde{u}_{\mid t=-\varepsilon}$ vanish for all $\varepsilon>0$. Therefore, Theorem 2.4 applied to the Cauchy problem for $L^{\sharp}$ with initial time $-\varepsilon$ implies that $\tilde{u}=0$ for all $(\tilde{t}, \tilde{x})$ such that $\tilde{t}<T_{0}$. Hence $u=0$ on a neighborhood of the origin.

\section{Application : a blow-up criterion for nonlinear equations}

\subsection{Statement of the result}

In coordinates $y=(t, x)$, we consider a nonlinear wave equation:

$$
\begin{aligned}
& \partial_{t}\left(a_{0}(u) \partial_{t} u\right)+\sum_{j=1}^{n} \partial_{t}\left(a_{j}(u) \partial_{x_{j}} u\right)+\partial_{x_{j}}\left(a_{j}(u) \partial_{t} u\right) \\
& \quad-\sum_{j, k=1}^{n} \partial_{x_{j}}\left(a_{j, k}(u) \partial_{x_{k}} u\right)+\partial_{t}\left(b_{0}(u)\right)+\sum_{j=1}^{n} \partial_{x_{j}}\left(b_{j}(u)\right)=F(u) .
\end{aligned}
$$

Assumption 6.1. - The coefficients are smooth functions of $u \in \mathbb{R}$. Morever, for all fixed $u$, the polynomial $a_{0} \tau^{2}+2 \sum a_{j} \tau \xi_{j}-\sum a_{j, k} \xi_{j} \xi_{k}$ is strictly hyperbolic in the direction $d t$.

The Cauchy problem for (6.1) with initial data

$$
u_{\mid t=0}=u_{0}, \quad \partial_{t} u_{\mid t=0}=u_{1},
$$

is well posed for $u_{0} \in H^{s}\left(\mathbb{R}^{n}\right)$ and $u_{1} \in H^{s-1}\left(\mathbb{R}^{n}\right)$ when $s>\frac{n}{2}+1$. The solution $u$ belongs to $C^{0}\left([0, T], H^{s}\right) \cap C^{1}\left([0, T] ; H^{s-1}\right)$. By uniqueness, there is a maximal time of existence $T^{*}$ and $u \in C^{0}\left(\left[0, T^{*}\left[; H^{s}\right) \cap C^{1}\left(\left[0, T^{*}\left[; H^{s-1}\right)\right.\right.\right.\right.$. Moreover, there is a classical blow-up criterion for the creation of singularities:

$4^{\mathrm{e}}$ SÉRIE - TOME $41-2008-\mathrm{N}^{\mathrm{o}} 2$ 
Theorem 6.2. - For $s>\frac{n}{2}+1$ and data $u_{0} \in H^{s}, u_{1} \in H^{s-1}$, if the maximal time of existence is finite, then

$$
\sup _{0 \leq t<T^{*}}\|u(t)\|_{L^{\infty}}+\left\|\partial_{t, x} u(t)\right\|_{L^{\infty}}=+\infty .
$$

See e.g. [1] for an extensive discussion of blow-up for solutions of wave equations or [9] for general first order quasilinear systems. Our goal is to show that one can replace the Lipschitz norm in (6.3) by a LL -norm.

Theorem 6.3. - For $s>\frac{n}{2}+1$ and data $u_{0} \in H^{s}, u_{1} \in H^{s-1}$, if $T^{*}<+\infty$, then

$$
\sup _{0 \leq t<T^{*}}\|u\|_{L^{\infty}\left([0, t] \times \mathbb{R}^{n}\right)}+\|u\|_{L L\left([0, t] \times \mathbb{R}^{n}\right)}=+\infty .
$$

The proof of Theorem 6.2 is based on the estimate :

Theorem 6.4. - For $s>\frac{n}{2}+1, M \in \mathbb{R}$ and $T_{0}>0$ given, there is a constant $C$, such that if $T \leq T_{0}$ and $u \in C^{0}\left(\left[0, T\left[; H^{s}\right) \cap C^{1}\left(\left[0, T\left[; H^{s-1}\right)\right.\right.\right.\right.$ is a solution of $(6.1)$ such that

$$
\sup _{0 \leq t<T}\|u(t)\|_{L^{\infty}}+\left\|\partial_{t, x} u(t)\right\|_{L^{\infty}} \leq M
$$

then

$$
\sup _{0 \leq t<T}\|u(t)\|_{H^{s}}+\left\|\partial_{t} u(t)\right\|_{H^{s-1}} \leq C\left(\left\|u_{0}\right\|_{H^{s}}+\left\|u_{1}\right\|_{H^{s-1}}\right) .
$$

Similarly, the proof of Theorem 6.3 is based on the following estimate :

Theorem 6.5. - For $s>\frac{n}{2}+1$ and $K \in \mathbb{R}$, there are constants $T_{1}>0, C_{1}$ and $\lambda$ such that if $u \in C^{0}\left(\left[0, T\left[; H^{s}\right) \cap C^{1}\left(\left[0, T\left[; H^{s-1}\right)\right.\right.\right.\right.$ is a solution of $(6.1)$ such that

$$
\sup _{0 \leq t<T}\|u\|_{L^{\infty}\left([0, t] \times \mathbb{R}^{n}\right)}+\|u\|_{L L\left([0, t] \times \mathbb{R}^{n}\right)} \leq K
$$

then, for $t<\min \left\{T, T_{1}\right\}$,

$$
\|u(t)\|_{H^{s-\lambda t}}+\left\|\partial_{t} u(t)\right\|_{H^{s-1-\lambda t}} \leq C_{1}\left(\left\|u_{0}\right\|_{H^{s}}+\left\|u_{1}\right\|_{H^{s-1}}\right) .
$$

Proof of Theorem 6.3 assuming Theorem 6.5. - It is sufficient to prove that if $u \in$ $C^{0}\left(\left[0, T\left[; H^{s}\right) \cap C^{1}\left(\left[0, T\left[; H^{s-1}\right)\right.\right.\right.\right.$ satisfies (6.7) then,

$$
\sup _{0 \leq t<T}\|u(t)\|_{H^{s}}+\left\|\partial_{t} u(t)\right\|_{H^{s-1}}<+\infty,
$$

implying that the solution can be continued after $T$.

Fix $\left.s_{1} \in\right] 1+\frac{n}{2}, s\left[\right.$. Decreasing $T_{1}$, we can assume that $T_{1} \leq\left(s-s_{1}\right) / \lambda$. Then (6.8) and the Sobolev imbedding theorem imply that

$$
\sup _{0 \leq t<T_{1}}\|u(t)\|_{L^{\infty}}+\left\|\partial_{t, x} u(t)\right\|_{L^{\infty}} \leq C(K)\left(\left\|u_{0}\right\|_{H^{s}}+\left\|u_{1}\right\|_{H^{s-1}}\right),
$$

where $C(K)$ depends only on $K$. Therefore, Theorem 6.4 implies that

$$
\sup _{0 \leq t<T_{1}}\|u(t)\|_{H^{s}}+\left\|\partial_{t} u(t)\right\|_{H^{s-1}} \leq C\left(K,\left\|u_{0}\right\|_{H^{s}},\left\|u_{1}\right\|_{H^{s-1}}\right) .
$$

The important point is that $T_{1}$ depends only on $K$. One can repeat the analysis for the Cauchy problem with initial time $T_{1}^{\prime}$ arbitrarily close to $T_{1}$, and after a finite number of iterations, this implies (6.9). 


\subsection{Proof of the nonlinear estimate}

We write the equation as a system

$$
\begin{gathered}
\partial_{t} v+\sum_{j=1}^{n} \partial_{x_{j}}\left(\tilde{a}_{j}(u) v\right)-\sum_{j, k=1}^{n} \partial_{x_{j}}\left(\tilde{a}_{j, k} \partial_{x_{k}} u\right)=-\sum_{j=1}^{n} \partial_{x_{j}}\left(\tilde{b}_{j}(u)\right)+F(u) \\
\partial_{t} u+\sum_{j=1}^{n} \tilde{a}_{j}(u) \partial_{x_{j}} u+\tilde{b}_{0}(u)=v / a_{0}
\end{gathered}
$$

with $\tilde{a}_{j}=a_{j} / a_{0}, \quad \tilde{a}_{j, k}=a_{j, k}+\tilde{a}_{j} a_{k}, \quad \tilde{b}_{0}=b_{0} / a_{0}, \quad \tilde{b}_{j}=b_{j}-a_{j} \tilde{b}_{0}$.

Introduce a dyadic partition of unity in space, $\sum \Delta_{k}\left(D_{x}\right)=\mathrm{Id}$, as in (3.3). The first step in the proof of Theorem 6.5 is an estimate of $\Delta_{k} \partial_{t} u$ and $\Delta_{k} v$ :

Proposition 6.6. - There is a constant $C(T, K)$ such that if $u \in C^{0}\left(\left[0, T\left[; H^{s}\right) \cap\right.\right.$ $C^{1}\left(\left[0, T\left[; H^{s-1}\right)\right.\right.$ is a solution of (6.1) which satisfies (6.7), then for all $k \geq 0$ and $t \in[0, T[$ :

$$
\left\|S_{k} \partial_{t} u(t)\right\|_{L^{\infty}}+\left\|S_{k} v(t)\right\|_{L^{\infty}} \leq C(T, K)(k+1) .
$$

This estimate is proved in the next subsection. Taking it for granted, we finish the proof of (6.8).

We use the para-differential calculus introduced in Section 3. The para-linearization procedure is based upon the following result.

Lemma 6.7. - Given $s>s_{1}>0$, there is a constant $C$ such that for $\sigma \in\left[s_{1}, s\right], a \in$ $H^{\sigma+\frac{1}{2} \log }\left(\mathbb{R}^{n}\right)$ and $v$ such that

$$
\sup _{k \geq 0}(k+1)^{-1}\left\|S_{k} v\right\|_{L^{\infty}} \leq K
$$

there holds av $-T_{a} v \in H^{\sigma-\frac{1}{2} \log }\left(\mathbb{R}^{n}\right)$ and

$$
\left\|a v-T_{a} v\right\|_{H^{\sigma-\frac{1}{2} \log }} \leq C K\|a\|_{H^{\sigma+\frac{1}{2} \log }} .
$$

Proof. - There holds $a v-T_{a} v=\sum w_{k}$ with $w_{k}=\Delta_{k} a S_{k+2} v$. The spectrum of $w_{k}$ is contained in the ball $\left\{|\xi| \leq 2^{k+4}\right\}$ and

$$
\left\|w_{k}\right\|_{L^{2}} \leq C K(k+1)^{\frac{1}{2}} 2^{-k s} \varepsilon_{k}\|a\|_{H^{s+\frac{1}{2} \log }}
$$

with $\left\{\varepsilon_{k}\right\}_{k}$ in the unit ball of $\ell^{2}$.

We also use the following nonlinear estimates :

Lemma 6.8. - Suppose that $u \in H^{\sigma+\frac{1}{2} \log }\left(\mathbb{R}^{n}\right) \cap L^{\infty}\left(\mathbb{R}^{n}\right)$ and a is a $C^{\infty}$ function on $\mathbb{R}$ such that $a(0)=0$. Then $a(u) \in H^{\sigma s+\frac{1}{2} \log }\left(\mathbb{R}^{n}\right)$ and

$$
\|a(u)\|_{H^{\sigma+\frac{1}{2} \log }} \leq C\left(\|u\|_{L^{\infty}}\right)\|a\|_{H^{\sigma+\frac{1}{2} \log }} .
$$


Proof of Theorem 6.5. - Consider a solution $u \in C^{0}\left(\left[0, T\left[; H^{s}\right) \cap C^{1}\left(\left[0, T\left[; H^{s-1}\right)\right.\right.\right.\right.$ of (6.1) satisfying (6.5). Fix $\left.s_{1} \in\right] \frac{d}{2}+1, s\left[\right.$. We show that there are constants $C$ and $C_{1}$, which depend only on $K$, such that for all $\lambda>0$ and $t \leq \min \left\{T, \frac{s-s_{1}}{\lambda}\right\}$ :

$$
\begin{aligned}
\|u(t)\|_{H^{s-\lambda t}}^{2}+\left\|\partial_{t} u(t)\right\|_{H^{s-1-\lambda t}}^{2} & \\
& +\lambda \int_{0}^{t}\left(\left\|u\left(t^{\prime}\right)\right\|_{H^{s-\lambda t+\frac{1}{2} \log }}^{2}+\left\|\partial_{t} u\left(t^{\prime}\right)\right\|_{H^{s-1-\lambda t+\frac{1}{2} \log }}^{2}\right) d t^{\prime} \\
\leq & C\left(\|u(0)\|_{H^{s}}^{2}+\left\|\partial_{t} u(0)\right\|_{H^{s-1}}^{2}\right) \\
& +C_{1} \int_{0}^{t}\left(\left\|u\left(t^{\prime}\right)\right\|_{H^{s-\lambda t+\frac{1}{2} \log }}^{2}+\left\|\partial_{t} u\left(t^{\prime}\right)\right\|_{H^{s-1-\lambda t+\frac{1}{2} \log }}^{2}\right) d t^{\prime} .
\end{aligned}
$$

Choosing $\lambda \geq C_{1}$, this implies (6.8).

a) We use the para-differential calculus as in Section 4. In addition to the quantization $T_{a}$ we use the modified operators $P_{a}^{\nu}$ (3.23). Using Corollary 3.12, we can fix $\nu$ and $\delta>0$ depending only on $K$, such that for all $t \in[0, T]$ and $w=\left(w_{1}, \ldots, w_{n}\right) \in C_{0}^{\infty}\left(\mathbb{R}^{n}\right)$ :

$$
\operatorname{Re} \sum_{j, k=1}^{n}\left(P_{b_{j, k}(t)}^{\nu} w_{k}, w_{j}\right)_{L^{2}} \geq \delta\|w\|_{L^{2}}^{2}
$$

with $b_{j, k}=a_{0}(u) \tilde{a}_{j, k}(u)$.

From now on we fix such a $\nu$ and use the notation $P_{b}$ in place of $P_{b}^{\nu}$. Lemma 3.10 can be extended to all values of $\sigma$ and there is a constant $C$ such that for all $t \in[0, T]$ and $\sigma \in\left[s_{1}, s\right]$ :

$$
\left\|\left(P_{a}-T_{a}\right) w\right\|_{H^{\sigma+1}} \leq C 2^{\nu}\|a\|_{L^{\infty}}\|w\|_{H^{\sigma}}
$$

Similarly

$$
\left\|\left(P_{a}-T_{a}\right) \partial_{x_{j}}\right\|_{H^{\sigma \pm \frac{1}{2} \log }} \leq C 2^{\nu}\|a\|_{L^{\infty}}\|w\|_{H^{\sigma \pm \frac{1}{2} \log }} .
$$

Using Proposition 3.3 for the spatial derivatives $\partial_{x_{j}} u$ and Proposition 6.6 for $v$, we deduce from the lemmas above that

$$
v=P_{a_{0}} \partial_{t} u+\sum_{j=1}^{n} P_{a_{j}} \partial_{x_{j}} u+g
$$

where $a_{j}$ stands for $a_{j}(u)$ and

$$
\left.\|g(t)\|_{H^{s-\lambda t-\frac{1}{2} \log } \leq C(K)} \leq\left\|\partial_{t} u(t)\right\|_{H^{s-1-\lambda t+\frac{1}{2} \log }}+\|u(t)\|_{H^{s-\lambda t+\frac{1}{2} \log }}\right) .
$$

In particular, this implies that

$$
\begin{aligned}
&\|v(t)\|_{H^{s-1-\lambda t}} \leq C(K)\left(\left\|\partial_{t} u(t)\right\|_{H^{s-1-\lambda t}}+\|u(t)\|_{H^{s-\lambda t}}\right) \\
&\|v(t)\|_{H^{s-1-\lambda t+\frac{1}{2} \log }} \leq C(K)\left(\left\|\partial_{t} u(t)\right\|_{H^{s-1-\lambda t+\frac{1}{2} \log }}+\|u(t)\|_{H^{s-\lambda t+\frac{1}{2} \log }}\right) .
\end{aligned}
$$

Similarly,

$$
\partial_{t} u+\sum_{j=1}^{n} P_{\tilde{a}_{j}} \partial_{x_{j}} u=P_{a_{0}^{-1}} v+g_{1}
$$


where $\tilde{a}_{j}=\tilde{a}_{j}(u)$ and

$$
\left\|g_{1}(t)\right\|_{H^{s-\lambda t-\frac{1}{2} \log }} \leq C(K)\left(\|u(t)\|_{H^{s-\lambda t+\frac{1}{2} \log }}+\|v(t)\|_{H^{s-1-\lambda t+\frac{1}{2} \log }}\right) .
$$

With (6.23), this implies that $g_{1}$ also satisfies an estimate similar to (6.21).

Another consequence is that

$$
\begin{aligned}
\left\|\partial_{t} u(t)\right\|_{H^{s-1-\lambda t}} & \leq C(K)\left(\|v(t)\|_{H^{s-1-\lambda t}}+\|u(t)\|_{H^{s-\lambda t}}\right), \\
\left\|\partial_{t} u(t)\right\|_{H^{s-1-\lambda t+\frac{1}{2} \log }} & \leq C(K)\left(\|v(t)\|_{H^{s-1-\lambda t+\frac{1}{2} \log }}+\|u(t)\|_{H^{s-\lambda t+\frac{1}{2} \log }}\right) .
\end{aligned}
$$

In the same vein,

$$
\partial_{t} v+\sum_{j=1}^{n} \partial_{x_{j}} P_{\tilde{a}_{j}} v-\sum_{j, k=1}^{n} \partial_{x_{j}} P_{\tilde{a}_{j, k}} \partial_{x_{k}} u=f
$$

with

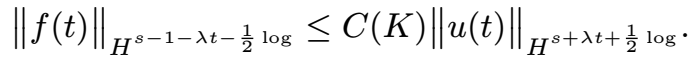

b) Multiply the equation (6.28) by $\left(1-\Delta_{x}\right)^{2(s-1-\lambda t)} v$ and integrate over $\mathbb{R}^{n}$. Using proposition 3.8 to bound the terms $\left(P_{\tilde{a}_{j}} \partial_{x_{j}} v,\left(1-\Delta_{x}\right)^{2(s-1-\lambda t)} v\right)_{L^{2}}$, implies that

$$
\begin{aligned}
& \frac{d}{d t}\|v(t)\|_{H^{s-1-\lambda t}}^{2}+2 \lambda\|v(t)\|_{H^{s-1-\lambda t+\frac{1}{2} \log }}^{2}-2 \operatorname{Re}\left(\sum_{j, k=1}^{n} \partial_{x_{j}} P_{\tilde{a}_{j, k}} \partial_{x_{k}} u,\left(1-\Delta_{x}\right)^{2(s-\lambda t)} v\right)_{L^{2}}
\end{aligned}
$$

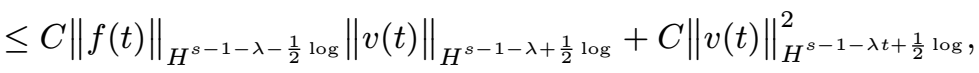

where $C$ depends only on $K$.

c) Multiply the equation (6.11) and $\left(1-\Delta_{x}\right)^{2(s-\lambda t)} \sum \partial_{x_{j}} P_{\tilde{a}_{j, k}} \partial_{x_{k}} u$. Using Propositions 3.8 and 3.9 ,

$$
\begin{aligned}
& -\operatorname{Re}\left(\sum_{j, k=1}^{n} \partial_{x_{j}} P_{\tilde{a}_{j, k}} \partial_{x_{k}} u,\left(1-\Delta_{x}\right)^{2(s-\lambda t)} v\right)_{L^{2}} \\
& =\operatorname{Re}\left(\sum_{j, k=1}^{n} P_{a_{0} \tilde{a}_{j, k}} \partial_{x_{k}} u,\left(1-\Delta_{x}\right)^{2(s-\lambda t)} \partial_{t} \partial_{x_{j}} u\right)_{L^{2}}+E
\end{aligned}
$$

where

$$
E(t) \leq C(K)\left(\left\|\partial_{t} u(t)\right\|_{H^{s-1-\lambda t+\frac{1}{2} \log }}^{2}+\|u(t)\|_{H^{s-\lambda t+\frac{1}{2} \log }}^{2}\right) .
$$

By Lemmas 6.10 and 6.11 below, the coefficients $b_{j, k}(u)=a_{0}(u) \tilde{a}_{j, k}(u)$ satisfy estimates similar to (6.12)

$$
\left\|S_{k} \partial_{t} b_{j, k}\right\|_{L^{\infty}} \leq(k+1) C(K) .
$$

Therefore $P_{\partial_{t} b_{j, k}}$ is of order $\log (D)$ and

$$
\begin{aligned}
2 \operatorname{Re}\left(\sum_{j, k=1}^{n} P_{a_{0} \tilde{a}_{j, k}} \partial_{x_{k}} u,\left(1-\Delta_{x}\right)^{2(s-\lambda t)} \partial_{t} \partial_{x_{j}} u\right)_{L^{2}} \\
=\frac{d}{d t} \operatorname{Re}\left(\sum_{j, k=1}^{n} P_{b_{j, k}} w_{k}, w_{j}\right)_{L^{2}}+2 \lambda\left(\sum_{j, k=1}^{n} P_{b_{j, k}} \tilde{w}_{k}, \tilde{w}_{j}\right)_{L^{2}}+E_{1}
\end{aligned}
$$


where $E_{1}$ satisfies an estimate similar to $E, w_{j}=\left(1-\Delta_{x}\right)^{(s-\lambda t)} \partial_{x_{j}} u$ and $\tilde{w}_{j}=(\ln (1-$ $\left.\left.\Delta_{x}\right)\right)^{\frac{1}{2}} w_{j}$.

Substituting these estimates in (6.30), integrating between 0 and $t$ and using (6.17) imply (6.16) and the theorem follows.

Notes on the proof of Theorem 6.4. - The proof of this theorem is quite similar, but much simpler, using the para-differential calculus with no logarithmic loss when the coefficients are Lipschitz continuous.

\subsection{Proof of Proposition 6.6}

For a $C^{1}$ function $a$ bounded with bounded derivatives on $[0, T] \times \mathbb{R}^{n}$, introduce the norm:

$$
\|a\|_{\mathcal{Z}}=\sup _{k \geq 0}(k+1)^{-1}\left\|S_{k}\left(D_{x}\right) a\right\|_{L^{\infty}\left([0, T] \times \mathbb{R}^{n}\right)} .
$$

Lemma 6.9. - There is a constant $C$ such that if $\mathbf{a}=\left\{a_{j}, a_{j, k}, b_{j}, c\right\}$ is a set of $C^{1} \cap W^{1, \infty}$ functions on $[0, T] \times \mathbb{R}^{n}$ satisfying

$$
\partial_{t}^{2} a_{0}=\sum_{j=1}^{d} \partial_{x_{j}}\left(\partial_{t} a_{j}+b_{j}\right)+\sum_{j, k=1}^{n} \partial_{x_{j}} \partial_{x_{k}} a_{j, k}+\partial_{t} b_{0}+c
$$

then

$$
\left\|\partial_{t} a_{0}\right\|_{\mathcal{Z}} \leq C\left(\|\mathbf{a}\|_{L^{\infty}\left([0, T] \times \mathbb{R}^{n}\right)}+\|\mathbf{a}\|_{L L\left([0, T] \times \mathbb{R}^{n}\right)}\right) .
$$

Proof. - a) Introducing a partition of unity, it is sufficient to prove the result when the functions are defined and compactly supported in $[0,+\infty[$ and $]-\infty, T]$. The two cases are similar, so we assume that the functions are defined for $t \geq 0$.

Consider the extension operator

$$
P_{0} a(t, x)=\left\{\begin{array}{lr}
a(t, x) & t \geq 0, \\
\alpha a(-t, x)+\beta a(-2 t, x)+\gamma a(-3 t, x), & t \leq 0
\end{array}\right.
$$

with

$$
\alpha+\beta+\gamma=1, \quad \alpha+2 \beta+3 \gamma=-1, \quad \alpha+4 \beta+9 \gamma=1,
$$

so that $P_{0} a, \partial_{t} P_{0} a$ and $\partial_{t}^{2} P_{0} a$ are continuous at $t=0$ when $a$ is $C^{2}$ on $\{t \geq 0\}$. Moreover

$$
\partial_{t} P_{0} a=P_{1} \partial_{t} a, \quad \partial_{t} P_{1} b=P_{2} \partial_{t} b,
$$

where $P_{1}$ and $P_{2}$ are similar extension operators. Then, the equation (6.33) can be extended to $\mathbb{R}^{1+n}$, with $P_{0} a_{0}$ in place of $a_{0}, P_{1} a_{j}$ in place of $a_{j}, P_{2} a_{j, k}$ in place of $a_{j, k}$, etc. Because the extensions operators preserve continuity at $t=0$, there is a constant $C$ such that

$$
\left\|P_{l} a\right\|_{L^{\infty}\left(\mathbb{R}^{1+d}\right)} \leq C\|a\|_{L^{\infty}(\{t \geq 0\})}, \quad\left\|P_{l} a\right\|_{L L\left(\mathbb{R}^{1+d}\right)} \leq C\|a\|_{L L(\{t \geq 0\})} .
$$

Hence it is sufficient to prove the lemma when the functions are defined on $\mathbb{R}^{1+d}$, which we now assume. 
b) In addition to the partition of unity $\operatorname{Id}=\sum \Delta_{k}\left(D_{x}\right)$ consider a similar partition of unity in time: $\operatorname{Id}=\sum \Delta_{p}^{\prime}\left(D_{t}\right)$. By Proposition 3.3,

$$
\left\|S_{p}^{\prime} S_{p} \partial_{t} a_{0}\right\|_{L^{\infty}\left(\mathbb{R}^{1+d}\right)} \leq C(p+1)\|a\|_{L L\left(\mathbb{R}^{1+d}\right)} .
$$

Similarly, for $q>p$ there holds

$$
\begin{aligned}
\left\|\Delta_{q}^{\prime} S_{p} \partial_{t} \partial_{x_{j}} a_{j}\right\|_{L^{\infty}} & \leq C(q+1) 2^{p}\left\|a_{j}\right\|_{L L}, \\
\left\|\Delta_{q}^{\prime} S_{p} \partial_{x_{k}} \partial_{x_{j}} a_{j_{k}}\right\|_{L^{\infty}} & \leq C(p+1) 2^{p}\left\|a_{j, k}\right\|_{L L} .
\end{aligned}
$$

Finally, using the equation (6.33) and similar estimates for the other functions, we see that for $q>p$ :

$$
\left\|\Delta_{q}^{\prime} S_{p} \partial_{t}^{2} a_{0}\right\|_{L^{\infty}\left(\mathbb{R}^{1+d}\right)} \leq C K q 2^{p}
$$

For $q>p$, the spectral localization of $\Delta_{q}^{\prime}$ implies that

$$
\left\|\Delta_{q}^{\prime} \partial_{t} S_{p} a_{0}\right\|_{L^{\infty}} \leq C 2^{-q}\left\|\Delta_{q}^{\prime} \partial_{t}^{2} S_{p} a_{0}\right\|_{L^{\infty}} \leq C K q 2^{p-q} .
$$

Therefore, writing that $S_{p} \partial_{t} a_{0}=S_{p}^{\prime} S_{p} \partial_{t} a_{0}+\sum_{q>p} \Delta_{q}^{\prime} S_{p} \partial_{t} a_{0}$ and adding the estimates above, one obtains (6.34).

To complete the proof of Proposition 6.6 we need the following estimates:

Lemma 6.10. - Let $F$ be s smooth function on $\mathbb{R}$ and let $a \in W^{1, \infty}\left([0, T] \times \mathbb{R}^{n}\right)$. Then $F(a) \in W^{1, \infty}\left([0, T] \times \mathbb{R}^{n}\right)$ and

$$
\|F(a)\|_{L L} \leq C\left(\|a\|_{L^{\infty}}\right)\|a\|_{L L} .
$$

Lemma 6.11. - Let $a \in W^{1, \infty}\left([0, T] \times \mathbb{R}^{n}\right)$ and $b \in L^{\infty}\left([0, T] \times \mathbb{R}^{d}\right)$. Then

$$
\|a b\|_{\mathcal{Z}} \leq C\left(\|a\|_{L^{\infty}}+\|a\|_{L L}\right)\|b\|_{\mathcal{Z}} .
$$

Proof. - The proof of (6.36) is immediate from the definition of the LL semi-norm.

To prove (6.37) write

$$
S_{k}(a b)=S_{k}\left(S_{k+2} a S_{k+4} b\right)+\sum_{p \geq k+3} \sum_{|q-p| \leq 2} S_{k}\left(\Delta_{p} a \Delta_{q} b\right) .
$$

The first term satisfies

$$
\left\|S_{k}\left(S_{k+2} a S_{k+4} b\right)\right\|_{L^{\infty}} \leq C\left\|S_{k+2} a\right\|_{L^{\infty}}\left\|S_{k+4} b\right\|_{L^{\infty}} \leq C(k+1)\|a\|_{L^{\infty}}\|b\|_{\mathcal{Z}} .
$$

Next, note that for $|p-q| \leq 2$,

$$
\left\|S_{k}\left(\Delta_{p} a \Delta_{q} b\right)\right\|_{L^{\infty}} \leq C\left\|\Delta_{p} a\right\|_{L^{\infty}}\left\|\Delta_{q} b\right\|_{L^{\infty}} \leq C(p+1)^{2} 2^{-p}\|a\|_{L L}\|b\|_{\mathcal{Z}}
$$

Adding up for $p \geq k+3$, this implies (6.37).

Proof of Proposition 6.6. - Let $A_{0}, A_{j}$ and $A_{j, k}$ be smooth functions on $\mathbb{R}$, vanishing at the origin, with derivative equal to $a_{0}, a_{j}$ and $a_{j, k}$ respectively. Then for $C^{0}\left([0, T] ; H^{s}\right) \cap$ $C^{1}\left([0, T] ; H^{s-1}\right)$ solutions the equation (6.1) reads:

$$
\begin{aligned}
\partial_{t}^{2} A_{0}(u) & +\sum_{j=1}^{n} 2 \partial_{t} \partial_{x_{j}} A_{j}(u)-\sum_{j, k=1}^{n} \partial_{x_{j}} \partial_{x_{k}} A_{j, k}(u) \partial_{t}\left(b_{0}(u)\right) \\
& +\sum_{j=1}^{n} \partial_{x_{j}}\left(b_{j}(u)\right)=F(u) .
\end{aligned}
$$


By Lemma 6.10, the $A_{0}(u), A_{j}(u)$ and $A_{j, k}(u)$ are $C^{1}$ and their $L^{\infty}$ and $L L$ norms are bounded by $C(K)$. Therefore, by Lemma 6.9 there is a constant $C(T, K)$ such that

$$
\left\|\partial_{t} A_{0}(u)\right\|_{\mathcal{Z}} \leq C(T, K)
$$

Since

$$
\partial_{t} u=\frac{1}{a_{0}(u)} \partial_{t} A_{0}(u)
$$

Lemma 6.11 implies that

$$
\left\|\partial_{t} u\right\|_{\mathcal{Z}} \leq C(T, K)
$$

Proposition 3.3 implies that

$$
\left\|\partial_{x_{j}} u\right\|_{\mathcal{Z}} \leq C\|u\|_{L L}
$$

Therefore, with Lemma 6.11 this implies that $v$ also satisfies the estimate (6.12) and the proof is now complete.

\section{REFERENCES}

[1] S. Alinhac, Blowup for nonlinear hyperbolic equations, Progress in Nonlinear Differential Equations and their Applications, 17, Birkhäuser, 1995.

[2] J.-M. Bony, Calcul symbolique et propagation des singularités pour les équations aux dérivées partielles non linéaires, Ann. Sci. École Norm. Sup. 14 (1981), 209-246.

[3] M. Cicognani, F. Colombini, Modulus of continuity of the coefficients and loss of derivatives in the strictly hyperbolic Cauchy problem, J. Differential Equations 221 (2006), 143-157.

[4] R. R. Coifman, Y. Meyer, Au delà des opérateurs pseudo-différentiels, Astérisque 57, Soc. Math. France, 1978.

[5] F. Colombini, E. De Giorgi, S. Spagnolo, Sur les équations hyperboliques avec des coefficients qui ne dépendent que du temps, Ann. Scuola Norm. Sup. Pisa Cl. Sci. 6 (1979), 511-559.

[6] F. Colombini, N. Lerner, Hyperbolic operators with non-Lipschitz coefficients, Duke Math. J. 77 (1995), 657-698.

[7] L. Hörmander, Linear partial differential operators, Die Grund. Math. Wiss., Bd. 116, Springer, 1963.

[8] E. Jannelli, Regularly hyperbolic systems and Gevrey classes, Ann. Mat. Pura Appl. 140 (1985), 133-145.

[9] A. MaJdA, Compressible fluid flow and systems of conservation laws in several space variables, Applied Mathematical Sciences 53, Springer, 1984.

[10] G. MÉTIVIER, Interaction de deux chocs pour un système de deux lois de conservation, en dimension deux d'espace, Trans. Amer. Math. Soc. 296 (1986), 431-479. 
[11] G. MéTIVIER, Small viscosity and boundary layer methods. Theory, stability analysis, and applications, Modeling and Simulation in Science, Engineering and Technology, Birkhäuser, 2004.

[12] G. Métivier, K. Zumbrun, Large viscous boundary layers for noncharacteristic nonlinear hyperbolic problems, Mem. Amer. Math. Soc. 175 (2005).

[13] Y. Meyer, Remarques sur un théorème de J.-M. Bony, in Proceedings of the Seminar on Harmonic Analysis (Pisa, 1980), Rend. Circ. Mat. Palermo (2), suppl. 1, 1981, $1-20$.

[14] T. Nishitani, Sur les équations hyperboliques à coefficients höldériens en $t$ et de classe de Gevrey en x, Bull. Sci. Math. 107 (1983), 113-138.

(Manuscrit reçu le 17 avril 2007;

accepté, après révision, le 31 janvier 2008.)

\author{
Ferruccio Colombini \\ Università di Pisa \\ Dipartimento di Matematica \\ Largo B. Pontecorvo 5 \\ 56127 Pisa, Italia \\ E-mail: Colombini@dm.unipi.it \\ Guy MÉTIVIER \\ Université de Bordeaux 1, IMB \\ 33405 Talence Cedex, France \\ E-mail: Guy .Metivier@math.u-bordeaux .fr
}

